\title{
Deformations and automorphisms: a framework for globalizing local tangent and obstruction spaces
}

\author{
BRIAN OSSERMAN
}

\begin{abstract}
Building on Schlessinger's work, we define a framework for studying geometric deformation problems which allows us to systematize the relationship between the local and global tangent and obstruction spaces of a deformation problem. Starting from Schlessinger's functors of Artin rings, we proceed in two steps: we replace functors to sets by categories fibered in groupoids, allowing us to keep track of automorphisms, and we work with deformation problems naturally associated to a scheme $X$, and which naturally localize on $X$, so that we can formalize the local behavior. The first step is already carried out by Rim in the context of his homogeneous groupoids, but we develop the theory substantially further. In this setting, many statements known for a range of specific deformation problems can be proved in full generality, under very general stack-like hypotheses.
\end{abstract}

Mathematics Subject Classification (2010): 14D15 (primary); 14D23 (secondary).

\section{Introduction}

Given a formal deformation problem, two of the most basic questions one can ask are: to what extent is it representable, and what are its tangent and obstruction spaces? In [18], Schlessinger gave an elementary and concise answer to the first question. The second question appears to be much more involved, with the most definitive work on the subject being Illusie's [11]. We propose a new framework which allows us to describe precisely the relationship between the global tangent and obstruction spaces and the local ones. While far less ambitious in scope, our approach is relatively elementary and allows us to treat a wide range of deformation problems uniformly and transparently. All the statements which we prove are well known in examples, but we show that they are in fact formal consequences of relatively mild hypotheses in a generality comparable to that of Schlessinger's work. As an added bonus, we are able to replace Schlessinger's conditions with more natural descent-theoretic ones, and we ultimately obtain results on the representing scheme.

This paper was partially supported by a fellowship from the National Science Foundation.

Received February 25, 2009; accepted in revised form October 15, 2009. 


\subsection{Statements}

A basic example of the sort of intuitive statement which we wish to be able to state as a general theorem is the following: if one has a deformation problem associated to a scheme $X$, which has a sheaf $\mathcal{A}$ on $X$ of infinitesimal automorphisms, and if (as in the case of deformations of smooth varieties, or vector bundles on a fixed variety) both deformations and obstructions are locally trivial, then the tangent space is $H^{1}(X, \mathcal{A})$, and obstructions lie naturally in $H^{2}(X, \mathcal{A})$. The idea is of course that we obtain our deformations by gluing together copies of the trivial deformation on open covers, and such gluings are controlled by $\mathcal{A}$.

In order to formalize such statements, we need to modify Schlessinger's context of functors of Artin rings in two ways. First, in order to be able to work with infinitesimal automorphisms, we are led to replace functors to sets with groupoids. Under additional stack-type gluing conditions on the deformation problem, we call such objects deformation stacks (note however that they are not strictly speaking stacks - see Section 2.2 and in particular Examples 2.20 and 2.21). Second, in Schlessinger's work there is no base scheme $X$ in the picture on which one can formulate the concepts of local or global. Accordingly, we consider problems associated to a scheme $X$, and which localize naturally on $X$ : i.e., instead of associating deformations to every Artin ring, we associate deformations to every pair $(U, A)$ of an open set on $X$ and an Artin ring. Under further gluing conditions, these objects will be called geometric deformation stacks, These will naturally carry two sheaves of $k$-vector spaces on $X: \mathcal{A}$, the sheaf of infinitesimal automorphisms, and $\mathcal{T}$, the sheaf of local first-order deformations.

We prove the following two theorems (see Section 1.3 below for a review of Schlessinger's terminology, and Section 3 for new definitions):

Theorem 1.1. Let $\mathcal{S}$ be a geometric deformation stack. Then the tangent space $T_{\mathcal{S}}$ of $\mathcal{S}$ fits into an exact sequence of $k$-vector spaces

$$
0 \rightarrow H^{1}(X, \mathcal{A}) \rightarrow T_{\mathcal{S}} \rightarrow H^{0}(X, \mathcal{T}) \rightarrow H^{2}(X, \mathcal{A})
$$

with the respective maps induced by inclusion of locally trivial deformations into $T_{\mathcal{S}}$, by passage from a deformation to the induced local deformation, and by formation of the obstruction to local isomorphisms between representatives of a local deformation inducing a well-defined gluing.

If we are given a local obstruction sheaf $\mathcal{O}$ b for $\mathcal{S}$, we have successive obstructions lying in $H^{0}(X, \mathcal{O} b), H^{1}(X, \mathcal{T})$, and $H^{2}(X, \mathcal{A}) / H^{0}(X, \mathcal{T})$, and measuring the obstructions to locally lifting objects, to choosing such lifts which are locally isomorphic, and to choosing local isomorphisms satisfying the cocycle condition, respectively.

Note that as a special case of the theorem, we obtain a precise version of our earlier assertion for the case that deformations and obstructions are locally trivial, when we can set $\mathcal{T}=\mathcal{O} b=0$; see Corollary 3.18.

As a consequence of Theorem 1.1, we draw conclusions about the existence and properties of a hull, under fairly general circumstances. 
Theorem 1.2. Let $\mathcal{S}$ be a geometric deformation stack on a scheme $X$. Then:

(i) the associated functor $F_{\left.\mathcal{S}_{(X,}\right)}$ satisfies Schlessinger's $(\mathrm{H} 1)$ and $(\mathrm{H} 2)$, and satisfies (H4) if and only if for each tiny extension $A^{\prime} \rightarrow A$ in $\operatorname{Art}(\Lambda, k)$, and each object $\eta \in A^{\prime}$, the natural map

$$
\operatorname{Aut}(\eta) \rightarrow \operatorname{Aut}\left(\left.\eta\right|_{A}\right)
$$

is surjective;

(ii) if $X$ is proper, and the sheaves $\mathcal{A}$ and $\mathcal{T}$ both carry the structure of coherent $\mathcal{O}_{X}$-modules, then $F_{\mathcal{S}_{(X,},}$ satisfies Schlessinger's $(\mathrm{H} 3)$, so has a hull $R$;

(iii) if further we have a local obstruction sheaf $\mathcal{O} b$ for $\mathcal{S}$, and it carries the structure of a coherent $\mathcal{O}_{X}$-module, then

$$
\begin{aligned}
h^{0}(X, \mathcal{T}) & +h^{1}(X, \mathcal{A})-h^{0}(X, \mathcal{O} b)-h^{1}(X, \mathcal{T})-h^{2}(X, \mathcal{A}) \\
\leq & \operatorname{dim} R-\operatorname{dim} \Lambda \leq \operatorname{dim} T_{\mathcal{S}},
\end{aligned}
$$

and if the first inequality is an equality and $\Lambda$ is regular, $R$ is a local complete intersection ring. If we have

$$
h^{0}(X, \mathcal{O} b)=h^{1}(X, \mathcal{T})=\operatorname{dim} T_{\mathcal{S}}+h^{2}(X, \mathcal{A})-h^{1}(X, \mathcal{A})-h^{0}(X, \mathcal{T})=0,
$$

then $R$ is smooth over $\Lambda$.

Here, a tiny extension is what Schlessinger called a small extension; see Definition 1.5 below. Although our conditions for a geometric deformation stack are formally stronger than Schlessinger's, it appears to be the case that (at least when the problem can be naturally defined for some $X$ and its open subsets) any deformation problem which satisfies (H1) and (H2) does so because it is associated to a geometric deformation stack, and that moreover one will verify the conditions for a geometric deformation stack in the process of checking (H1) and (H2). This is born out by a number of examples, treated in Section 2.3 below.

We also mention that in the locally unobstructed case, the form of the tangent and obstruction spaces are such that one expects that they arise as the hypercohomology of a two-term complex. We explore this further in [16].

Although this work is self-contained and was developed largely independently, it is closely related to work of Grothendieck and Rim as follows: Grothendieck's work on Exal in [7] laid the framework for treating deformations from a groupoid point of view, and exploiting certain additive structures on categories in order to do so. Meanwhile, Rim's theory of homogeneous groupoids [17], which is formally equivalent to our deformation stacks, began the process of treating deformation problems systematically in the context of groupoids. However, Rim's general work was focused primarily on further development of Schlessinger's criterion, and on criteria for the existence of smooth presentations of groupoids, so the actual overlap of results with the present paper is miminal. Indeed, the bulk of the present paper is Section 2, which develops the theory of deformation stacks further, and 
may be viewed as synthesizing and expanding on the ideas of Grothendieck and Rim, using categorical torsor structures to prove very general statements on the structure of liftings of objects and automorphisms over small ring extensions. Finally, it should be emphasized that everything we do has been very well known in specific examples for some time; our main contribution is to provide a framework in which the arguments can be systematized, so that they apply formally to many deformations problems at once. Schlessinger's original paper was a valuable source of such statements made in more specific contexts, as was the book [9] of Robin Hartshorne.

\section{A word on the use of stacks}

We wish to underline that although we use concepts from the theory of stacks, no reader should be intimidated by this fact; indeed, we make no use of algebraic stacks or the étale topology, and one should think of stacks in this context as being nothing more than functors which remember automorphisms and satisfy certain natural gluing conditions (which we will restate below). In fact, we use no results at all from the theory of stacks, and our presentation is entirely elementary and self contained.

\subsection{Notation and conventions}

Given a field $k$, and a complete Noetherian ring $\Lambda$ with residue field $k$, we denote by $\operatorname{Art}(\Lambda, k)$ the category of local Artin $\Lambda$-algebras with residue field $k$. We will use the notational convention that $\epsilon$ is always a square-zero element. For any $k$-vector space $V$, we will also denote by $k[V]$ the algebra having additive group $k \oplus V$, with square-zero multiplication for elements of $V$. Both $k[\epsilon]$ and $k[V]$ always denote rings endowed with the "trivial" $\Lambda$-algebra structure (i.e., the one factoring through $\Lambda \rightarrow k)$. Finally, for any $A \in \operatorname{Art}(\Lambda, k)$ we denote by $\pi: A \rightarrow k$ the residue field map.

$\Lambda$ is frequently either $k$ or, if $k$ is perfect of characteristic $p$, and one wants to work in mixed characteristic, the Witt vectors $W(k)$. The latter case is universal: every complete Noetherian local ring with residue field $k$ is canonically an algebra over $W(k)$ (see [19, Proposition 10 of II, Section 5]). When working with families over a base space, one often takes $\Lambda$ to be a complete local ring of the base.

\subsection{Review of Schlessinger}

For the convenience of the reader and in order to assign terminology intended to motivate the relationship to deformation stacks, we briefly review Schlessinger's criterion. Schlessinger considered functors of the following type.

Definition 1.3. A covariant functor $F: \operatorname{Art}(\Lambda, k) \rightarrow$ Set is a predeformation functor if $F(k)$ consists of a single element. If $F$ is a predeformation functor, we say that $T_{F}:=F(k[\epsilon])$ is its tangent space. 
Notation 1.4. Given a predeformation functor $F$, we use $\zeta_{0}$ to denote the unique object of $F(k)$, and $\zeta_{V}$ (respectively, $\zeta_{\epsilon}$ ) to denote the object induced on $k[V]$ (respectively, $k[\epsilon])$ by $\zeta_{0}$ under the structure map.

Given a morphism $f: A \rightarrow A^{\prime}$ in $\operatorname{Art}(\Lambda, k)$, and an object $\eta \in F(A)$, we will denote the object of $F\left(A^{\prime}\right)$ induced by $\eta$ under $f$ by $f_{*}(\eta)$ or, when there is no ambiguity, by $\left.\eta\right|_{A^{\prime}}$.

We will deviate slightly from Schlessinger's terminology.

Definition 1.5. A surjection $p: A^{\prime} \rightarrow A$ in $\operatorname{Art}(\Lambda, k)$ with kernel $I$ is small if $I \cdot \mathfrak{m}_{A^{\prime}}=0$. We say that $p$ is tiny if it is small, and if further $I$ is a principal ideal.

Note that the kernel of a small extension is necessarily square-zero and a $k$ vector space, and that a tiny extension has kernel isomorphic to $k$. Schlessinger used "small" for what we call "tiny"; our usage of "small" follows Huybrechts and Lehn [10, 2.A.5]), as well as Fantechi and Göttsche [5, Definition 6.1.9]). The more general notion of small is important for certain applications of obstruction theory; see in particular Theorem 3.14 below.

Given morphisms $A^{\prime} \rightarrow A, A^{\prime \prime} \rightarrow A$ in $\operatorname{Art}(\Lambda, k)$, there is a natural map

$$
\mathcal{F}\left(A^{\prime} \times{ }_{A} A^{\prime \prime}\right) \rightarrow \mathcal{F}\left(A^{\prime}\right) \times \mathcal{F}(A) \mathcal{F}\left(A^{\prime \prime}\right) .
$$

Schlessinger's conditions are the following:

(H1) The map (1.1) is surjective whenever $A^{\prime \prime} \rightarrow A$ is a tiny extension.

(H2) The map (1.1) is a bijection when $A=k$, and $A^{\prime \prime}=k[\epsilon]$.

(H3) The tangent space $T_{F}$ is finite-dimensional over $k$.

(H4) The map (1.1) is a bijection whenever $A^{\prime \prime}=A^{\prime}$ and $A^{\prime} \rightarrow A$ is a tiny extension.

We remark that if $(\mathrm{H} 2)$ is satisfied, then $T_{F}$ can be naturally given the structure of a $k$-vector space. We further remark that every reasonable deformation problem seems to satisfy (H1) and (H2), while (H3) is typically satisfied where proper schemes are involved, and (H4) is a substantially stronger condition, closely tied to the behavior of automorphisms. We therefore make the following definition.

Definition 1.6. We say that a predeformation functor $F$ is a deformation functor if it satisfies Schlessinger's (H1) and (H2).

Denote by $\widehat{\operatorname{Art}}(\Lambda, k)$ the category of complete Noetherian local $\Lambda$-algebras with residue field $k$. We recall that a predeformation functor $F$ may be extended to a functor $\hat{F}: \widehat{\operatorname{Art}}(\Lambda, k) \rightarrow$ Set, simply by taking the appropriate limits over all $R / \mathfrak{m}_{R}^{n}$ for $R \in \widehat{\operatorname{Art}}(\Lambda, k)$. We recall the following basic definitions, the first one a direct extension of the notion of formal smoothness to morphisms of functors:

Definition 1.7. If $F, F^{\prime}: \operatorname{Art}(\Lambda, k) \rightarrow$ Set are functors, and we have a morphism $\varphi: F \rightarrow F^{\prime}$, we say that $\varphi$ is formally smooth if for every surjective map $A^{\prime} \rightarrow A$ in $\operatorname{Art}(\Lambda, k)$, the canonical map

$$
F\left(A^{\prime}\right) \rightarrow F(A) \times F^{\prime}(A) F^{\prime}\left(A^{\prime}\right)
$$

is surjective. 
The notions of representability are the following:

Definition 1.8. We say that $F: \operatorname{Art}(\Lambda, k) \rightarrow$ Set is prorepresentable if there is a pair $(R, \xi)$, with $R \in \widehat{\operatorname{Art}}(\Lambda, k)$, and $\xi \in \hat{F}(R)$, such that the induced map $h_{R} \mid \operatorname{Art}(\Lambda, k) \rightarrow F$ is an isomorphism of functors.

We say that a pair $(R, \xi)$ is a hull of $F$ if the map $\left.h_{R}\right|_{\operatorname{Art}(\Lambda, k)} \rightarrow F$ is formally smooth, and induces an isomorphism $h_{R}(k[\epsilon]) \stackrel{\sim}{\rightarrow} F(k[\epsilon])$.

Schlessinger's basic theorem is as follows:

Theorem 1.9 (Schlessinger, Theorem 2.11 of [18]). Let $F$ be a predeformation functor. Then $F$ has a hull if and only if $F$ satisfies (H1), (H2), and (H3). Moreover, $F$ is prorepresentable if and only if it satisfies in addition (H4).

ACKNOWLEDGEMENTS. I would like to thank David Eisenbud, Ravi Vakil, Charles Weibel, Robin Hartshorne, and Luc Illusie, and particularly Martin Olsson and Max Lieblich, for many patient and illuminating conversations.

\section{Deformation stacks}

We begin with a simple translation of Schlessinger's work on deformation functors into a more stack-theoretic language, which, while imposing potentially stricter hypotheses, gives a more complete picture of the situation. This is equivalent to Rim's homogeneous groupoids, but our definition is more motivated by the descent conditions of stack theory, and we examine this relationship more closely than is required to prove our main results. We then proceed to give a number of examples of deformation problems which naturally form deformation stacks, and finally to prove several technical results which ultimately play an important role in proving our main theorems. The main theorem in this direction, which is vital to understanding obstructions, is that with no additional hypotheses, liftings of objects and automorphisms over small extensions can be studied in terms of the tangent space $T_{\mathcal{S}}$ and the infinitesimal automorphism group $A_{\mathcal{S}}$ respectively.

\subsection{Definitions}

To avoid technical issues, we assume throughout that we work with small categories, so that every category has an associated set of isomorphism classes. This can be accomplished by, for instance, working in a fixed universe in the sense of Grothendieck. In order to keep our presentation self-contained and to fix terminology, we begin by recalling the basic definitions relating to categories fibered in groupoids.

Definition 2.1. Fix a category $C$. We say that a category $\mathcal{S}$, together with a (covariant) functor to $C$, is a category fibered in groupoids over $C$, if: 
(i) Given a morphism $T \rightarrow T^{\prime}$ in $C$, and an object $\eta^{\prime}$ in $\mathcal{S}$ over $T^{\prime}$, there exists $\eta$ in $\mathcal{S}$ over $T$ and a morphism $\eta \rightarrow \eta^{\prime}$ over the given morphism $T \rightarrow T^{\prime}$.

(ii) Given a diagram with the solid arrows below:

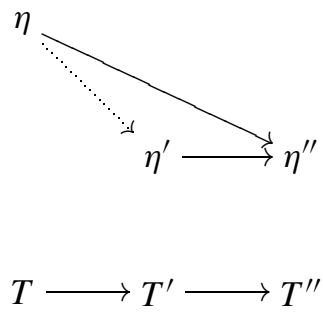

the dotted arrow, making the triangle commute, exists and is unique.

The terminology is justified as follows: given a functor $\mathcal{S} \rightarrow C$, and an object $T \in C$, we define the fiber category $\mathcal{S}_{T}$ to consist of the objects of $\mathcal{S}$ lying over $T$, together with the morphisms lying over the identity morphism $T \rightarrow T$. It then follows from (ii) above that every fiber category $\mathcal{S}_{T}$ of a category fibered in groupoids $\mathcal{S}$ is indeed a groupoid, in the sense that every morphism is an isomorphism.

An observation which we will use implicitly many times when checking compatibility of definitions is the following, which is an immediate consequence of the definitions.

Proposition 2.2. Let $\mathcal{S} \rightarrow C$ be a category fibered in groupoids. Then every morphism $f: \eta^{\prime} \rightarrow \eta^{\prime \prime}$ in $\mathcal{S}$, lying over $T^{\prime} \rightarrow T^{\prime \prime}$ in $C$, is a "relative monomorphism," in the sense that if $g_{1}, g_{2}: \eta \rightarrow \eta^{\prime}$ both lie over a given $T \rightarrow T^{\prime}$ in $C$, and $f \circ g_{1}=f \circ g_{2}$, then $g_{1}=g_{2}$.

The definition of a category fibered in groupoids implicitly incorporates notions of pullbacks of objects and morphisms. We begin with objects.

Definition 2.3. Suppose we are given a morphism $f: T \rightarrow T^{\prime}$ in $C$, and $\eta^{\prime} \in \mathcal{S}_{T^{\prime}}$. The pair of $\eta$ and the morphism $\eta \rightarrow \eta^{\prime}$ given by (i) above is the pullback of $\eta^{\prime}$ under $f$, which we denote by $f^{*} \eta^{\prime}$, or $\left.\eta^{\prime}\right|_{T}$.

By (ii) above we immediately see the following uniqueness statement.

Proposition 2.4. A pullback $f^{*}\left(\eta^{\prime}\right)$ is unique up to unique isomorphism.

We emphasize that although we use object notation for pullbacks of objects, in order to obtain the above uniqueness it is necessary to include also the data of the morphism $\eta \rightarrow \eta^{\prime}$. Even with this data, pullbacks are not uniquely defined in an absolute sense, but because they are unique up to unique isomorphism, in practice they may often be treated as uniquely defined. For instance, we may canonically identify morphisms to or from any two pullbacks of a given object. Sometimes we are required to explicitly consider the "unique isomorphism" between two pullbacks, as in the discussion of the cocycle condition below.

We can also define pullbacks of morphisms. 
Definition 2.5. Suppose we are given a morphism $f: T \rightarrow T^{\prime}$ in $C$. If we have two objects $\eta, \eta^{\prime} \in S_{T^{\prime}}$, and a morphism $\varphi: \eta \rightarrow \eta^{\prime}$ over the identity morphism of $T^{\prime}$, we also have the pullback $f^{*}(\varphi): f^{*}(\eta) \rightarrow f^{*}\left(\eta^{\prime}\right)$ of $\varphi$ under $f$, a morphism lying over the identity morphism of $T$. This pullback, also sometimes denoted by $\left.\varphi\right|_{T^{\prime}}$, is defined as the morphism obtained by applying (ii) above to the composed morphism $f^{*}(\eta) \rightarrow \eta \stackrel{\varphi}{\rightarrow} \eta^{\prime}$ and $f^{*}\left(\eta^{\prime}\right) \rightarrow \eta^{\prime}$.

The following basic compatibility properties follow from the definitions. More specifically, the first is immediate from (i), while the rest may be checked using the uniqueness hypothesis in (ii) of the definition of a category fibered in groupoids.

Proposition 2.6. We have:

(i) The pullback of a pullback is a pullback: given $T \rightarrow T^{\prime} \rightarrow T^{\prime \prime}$ in $C$, and $\eta \in S_{T^{\prime \prime}}$, then $\left.\left(\left.\eta\right|_{T^{\prime}}\right)\right|_{T}$ is a pullback of $\eta$ under the composition $T \rightarrow T^{\prime \prime}$.

(ii) The isomorphisms between different pullbacks satisfy the cocycle condition: given $T \rightarrow T^{\prime}$ and $\eta \in S_{T^{\prime}}$, suppose that $\eta_{1}, \eta_{2}, \eta_{3}$ are three different pullbacks of $\eta$ to $T$, and for each $i, j$ let $\varphi_{i, j}: \eta_{i} \stackrel{\sim}{\rightarrow} \eta_{j}$ be the unique isomorphism identifying $\eta_{i}$ with $\eta_{j}$. Then these satisfy the cocycle condition $\varphi_{2,3} \circ \varphi_{1,2}=\varphi_{1,3}$.

(iii) Pullback of morphisms commutes with composition: given $T \stackrel{f}{\rightarrow} T^{\prime} \stackrel{g}{\rightarrow} T^{\prime \prime}$, and $\eta, \eta^{\prime}$ in $\mathcal{S}_{T^{\prime \prime}}$, and a morphism $\varphi: \eta \rightarrow \eta^{\prime}$ lying over the identity morphism of $T^{\prime \prime}$, we have $f^{*}\left(g^{*}(\varphi)\right)=(f \circ g)^{*}(\varphi)$.

(iv) Pullback of morphisms commutes with composition: given $f: T \rightarrow T^{\prime}$, and $\eta, \eta^{\prime}, \eta^{\prime \prime}$ in $\mathcal{S}_{T^{\prime}}$, and two morphisms $\eta \stackrel{\varphi}{\rightarrow} \eta^{\prime} \stackrel{\varphi^{\prime}}{\rightarrow} \eta^{\prime \prime}$ lying over the identity morphism of $T^{\prime}$, we have $f^{*}\left(\varphi^{\prime} \circ \varphi\right)=f^{*}\left(\varphi^{\prime}\right) \circ f^{*}(\varphi)$.

We now give a definition of deformation stack which is very close to (and indeed, equivalent to; see Remark 2.8 below) Rim's definition of homogeneous groupoid. For a definition which places deformation stacks more visibly in the context of standard stack conditions, see Section 2.2 below.

Definition 2.7. A category $\mathcal{S}$ fibered in groupoids over $\operatorname{Art}(\Lambda, k)^{\text {opp }}$ is a deformation stack if the fiber of $\mathcal{S}$ over $k$ is the "trivial" groupoid: i.e., there exists a unique morphism between any two objects; and if for every square of the form

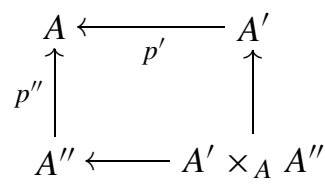

in $\operatorname{Art}(\Lambda, k)$ with $p^{\prime \prime}$ surjective, we have:

(i) ("morphisms form a sheaf") Given objects $\eta, \eta^{\prime}$ in $\mathcal{S}$ over $A^{\prime} \times{ }_{A} A^{\prime \prime}$, the natural map

$$
\operatorname{Mor}\left(\eta, \eta^{\prime}\right) \rightarrow \operatorname{Mor}\left(\left.\eta\right|_{A^{\prime}},\left.\eta^{\prime}\right|_{A^{\prime}}\right) \times_{\operatorname{Mor}\left(\left.\eta\right|_{A},\left.\eta^{\prime}\right|_{A}\right)} \operatorname{Mor}\left(\left.\eta\right|_{A^{\prime \prime}},\left.\eta^{\prime}\right|_{A^{\prime \prime}}\right)
$$


is a bijection. Here all sets of morphisms are taken in the appropriate fiber categories, or equivalently, are assumed to lie over the identity morphism of the underlying Artin ring.

(ii) ("objects satisfy effective descent") Given objects $\eta^{\prime}, \eta^{\prime \prime}$ in $\mathcal{S}$ over $A^{\prime}$ and $A^{\prime \prime}$ respectively, and a morphism $\varphi:\left.\left.\eta^{\prime}\right|_{A} \rightarrow \eta^{\prime \prime}\right|_{A}$ over $\operatorname{id}_{A}$, there exists an object $\tilde{\eta}$ over $A^{\prime} \times_{A} A^{\prime \prime}$ such that $\eta^{\prime}=\left.\tilde{\eta}\right|_{A^{\prime}}, \eta^{\prime \prime}=\left.\tilde{\eta}\right|_{A^{\prime \prime}}$, and $\varphi$ is the unique isomorphism identifying both $\left.\eta^{\prime}\right|_{A}$ and $\left.\eta^{\prime \prime}\right|_{A}$ as pullbacks of $\tilde{\eta}$ to $A$.

Remark 2.8. The definition of a deformation stack can be stated more compactly by requiring that the groupoid version of the natural map (1.1) be an equivalence of categories. This is equivalent to our stack conditions because the conditions on morphisms and objects are equivalent to the natural functor being fully faithful and essentially surjective, respectively. Thus, the definition we have given of deformation stack is equivalent to Rim's homogeneous groupoids ([17, Definition 2.5 and Remark 2.6 (b)]).

To avoid unnecessary obfuscation involving the use of the opposite category, when working with a deformation stack $\mathcal{S}$, we will work freely with ring morphisms and pushforward of objects of $\mathcal{S}$. Note the slightly confusing situation that the direction of a pushforward morphism in $\mathcal{S}$ will be opposite to the direction of the ring homomorphism in $\operatorname{Art}(\Lambda, k)$ over which it lies.

Notation 2.9. We still use the notation $\zeta_{0}, \zeta_{V}$, and $\zeta_{\epsilon}$ in the context of deformation stacks as in Notation 1.4; we assume we have fixed a choice of object $\zeta_{0}$, and of pushforwards $\zeta_{V}$ and $\zeta_{\epsilon}$.

Although these are not uniquely-defined objects, they are unique up to unique isomorphism. Note that more generally, the definition of a category fibered in groupoids together with the hypothesis that $\mathcal{S}_{k}$ is trivial implies that every object $\eta$ in $\mathcal{S}$ has a unique morphism $\zeta_{0} \rightarrow \eta$.

To each deformation stack $\mathcal{S}$, we have the associated functor of isomorphism classes $F_{\mathcal{S}}$. By virtue of the hypothesis that $\mathcal{S}_{k}$ is trivial, $F_{\mathcal{S}}$ is a predeformation functor, and it is easy to check that conditions (i) and (ii) above imply:

Proposition 2.10. Suppose $F_{\mathcal{S}}$ is the functor associated to a deformation stack. Then $F_{\mathcal{S}}$ is a deformation functor.

Our philosophy is that although being associated to a deformation stack is in a literal sense stronger than being a deformation functor, we expect that "in nature" any deformation functor is in fact the functor associated to a deformation stack, and that furthermore in any given case, the proof that it is a deformation functor will include a proof that the natural groupoid is a deformation stack. As evidence for this philosophy, we have the following simple rephrasing of Olsson [15, Lemma 1.4.4]:

Proposition 2.11. Suppose $\mathcal{S}$ is a deformation problem obtained via restriction around a point of an algebraic stack. Then $\mathcal{S}$ is a deformation stack. 
However, deformation stacks are far more general, arising for instance from deformations of any scheme (without any polarization, and without properness hypotheses), as well as in a variety of other contexts, discussed in Section 2.3 below.

Moving beyond Schlessinger's conditions (H1) and (H2), another advantage of the stack perspective is a sharp understanding of (H4), already observed (in necessarily imprecise form) by Schlessinger in [18, Remark 2.15], and made more precise by Rim in [17, Proposition 2.7].

Proposition 2.12. For every surjection $A \rightarrow B$ in $\operatorname{Art}(\Lambda, k)$, we have that the natural map

$$
F_{\mathcal{S}}\left(A \times_{B} A\right) \rightarrow F_{\mathcal{S}}(A) \times F_{\mathcal{S}}(B) F_{\mathcal{S}}(A)
$$

is bijective if and only if for every object $\eta \in \mathcal{S}_{A}$, the natural map from $\operatorname{Aut}(\eta)$ to $\operatorname{Aut}\left(\left.\eta\right|_{B}\right)$ is surjective.

In particular, $F_{\mathcal{S}}$ satisfies Schlessinger's (H4) if and only if every automorphism can be extended over any tiny extension.

Proof. Fix $A \rightarrow B$ a surjection in $\operatorname{Art}(\Lambda, k)$, and an object $\eta \in \mathcal{S}_{A}$. The stack conditions on $\mathcal{S}$ given in Definition 2.7 mean that given objects $\eta, \eta^{\prime} \in \mathcal{S}_{A}$ such that $\left.\left.\eta\right|_{B} \cong \eta^{\prime}\right|_{B}$, fixing an isomorphism gives a bijection between isomorphism classes of objects $\tilde{\eta} \in \mathcal{S}_{A \times{ }_{B} A}$ such that $\tilde{\eta}$ restricts to $\eta$ and $\eta^{\prime}$ under the two projection maps, and $\operatorname{Aut}\left(\left.\eta\right|_{B}\right) /\left\langle\left.\operatorname{Aut}(\eta)\right|_{B},\left.\operatorname{Aut}\left(\eta^{\prime}\right)\right|_{B}\right\rangle$.

In particular, if $\operatorname{Aut}(\eta) \rightarrow \operatorname{Aut}\left(\left.\eta\right|_{B}\right)$ and $\operatorname{Aut}\left(\eta^{\prime}\right) \rightarrow \operatorname{Aut}\left(\left.\eta^{\prime}\right|_{B}\right)$, we have that $\tilde{\eta}$ is uniquely determined by $\eta$ and $\eta^{\prime}$, so we have the asserted bijectivity for the natural map (2.1). Conversely, if we consider the case $\eta=\eta^{\prime}$, bijectivity of (2.1) is equivalent to uniqueness of $\tilde{\eta}$, which implies that $\operatorname{Aut}(\eta)$ surjects onto $\operatorname{Aut}\left(\left.\eta\right|_{B}\right)$.

Thus, Schlessinger's conditions (H1), (H2), and (H4) all have very natural interpretations in the context of deformation stacks. We will ultimately prove the statement asserted in Theorem 1.2 that there is a simple sufficient condition on a (geometric) deformation stack to obtain (H3) as well.

We conclude with definitions of automorphism, tangent and obstruction spaces for deformation stacks. While the last two may be defined in terms of the associated functors, of course the automorphism space is not. In fact, we work with successive obstruction spaces: the intuition for successive obstructions taking value in vector spaces $V_{1}, \ldots, V_{n}$ is that given a small extension $A^{\prime} \rightarrow A$ with kernel $I$, and an object $\eta$ over $A$, we obtain an element of $V_{1} \otimes_{k} I$ giving a "first obstruction" to lifting $\eta$ to $A^{\prime}$, and if that element vanishes, we have an element of $V_{2} \otimes_{k} I$ giving a "second obstruction," and so forth, and there exists a lift of $\eta$ to $A^{\prime}$ if and only if every obstruction vanishes. However, for the sake of simplicity we make a definition focusing on the first non-zero obstruction.

Definition 2.13. The infinitesimal automorphism group $A_{\mathcal{S}}$ of $\mathcal{S}$ is the group of automorphisms of $\zeta_{\epsilon}$ in $\mathcal{S}_{k[\epsilon]}$.

The tangent space $T_{\mathcal{S}}$ of $\mathcal{S}$ is defined to be $T_{F_{\mathcal{S}}}$, the tangent space of the associated deformation functor. 
Given $k$-vector spaces $V_{1}, \ldots, V_{n}$, a successive obstruction theory for $\mathcal{S}$ taking values in $V_{1}, \ldots, V_{n}$ consists of the data, for each small extension $A^{\prime} \rightarrow A$ in $\operatorname{Art}(\Lambda, k)$ with kernel $I$, and $\eta \in \mathcal{S}_{A}$, of an $m \in\{1, \ldots, n\}$ and an element $\mathrm{ob}_{\eta, A^{\prime}} \in V_{m} \otimes_{k} I$ such that:

(i) $\mathrm{ob}_{\eta, A^{\prime}} \neq 0$ unless $m=n$;

(ii) there exists $\eta^{\prime} \in \mathcal{S}_{A^{\prime}}$ such that $\left.\eta^{\prime}\right|_{A}=\eta$ if and only if $m=n$ and $\mathrm{ob}_{\eta, A^{\prime}}=0$.

Furthermore, we impose the following functoriality condition: suppose we have another small extension $B^{\prime} \rightarrow B$ with kernel $J$, and $\varphi: A^{\prime} \rightarrow B^{\prime}$ such that $\varphi(I) \subseteq$ $J$, so that $\varphi$ also induces maps $I \rightarrow J$ and $A \rightarrow B$. Then we require that $\mathrm{ob}_{\left.\eta\right|_{B}, B^{\prime}}=$ $\varphi\left(\mathrm{ob}_{\eta, A^{\prime}}\right)$ if the latter is non-zero or if $m=n$, and otherwise $\mathrm{ob}_{\left.\eta\right|_{B}, B^{\prime}} \in V_{m^{\prime}} \otimes_{k} J$ for $m^{\prime}>m$.

In the special case that $n=1$, we say that $V_{1}$ is an obstruction space for $\mathcal{S}$.

While $A_{\mathcal{S}}$ is a priori only a group, we will see in Corollary 2.38 below that it is actually also a $k$-vector space, with addition agreeing with composition.

\subsection{Deformations stacks and stack conditions}

For those familiar with stack theory, particularly as it is typically used in moduli space theory, our definition of deformation stack has little to do with any usual notion of stack. We now clarify the situation by giving an equivalent definition of deformation stack which is stated as a standard stack condition, but applied to a collection of covers which do not satisfy the hypotheses of a Grothendieck (pre)topology. This discussion may be considered purely philosophical, and will not be used in anything which follows.

The first step is to rephrase the squares of Definition 2.7 in a more visibly descent-theoretic form.

Definition 2.14. We say a square

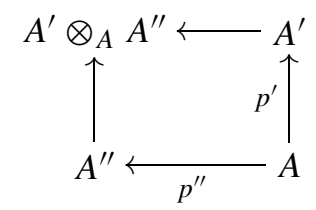

in $\operatorname{Art}(\Lambda, k)$ is a Schlessinger square if the following conditions are satisfied:

(i) $A \rightarrow A^{\prime} \times A^{\prime \prime}$ is injective;

(ii) $p^{\prime}$ is surjective;

(iii) $p^{\prime \prime}\left(\operatorname{ker} p^{\prime}\right)$ is closed under multiplication in $A^{\prime \prime}$.

From a scheme theory point of view, the first condition says that $\operatorname{Spec} A^{\prime} \amalg \operatorname{Spec} A^{\prime \prime}$ maps scheme-theoretically surjectively to $\operatorname{Spec} A$, while the second of course means that $\operatorname{Spec} A^{\prime} \rightarrow \operatorname{Spec} A$ is a closed immersion. However, the third condition remains somewhat mysterious, from a geometric point of view. Nonetheless, given a 
Schlessinger square, it makes geometric sense to think of $A^{\prime}$ and $A^{\prime \prime}$ as covering $A$, with $A^{\prime} \otimes_{A} A^{\prime \prime}$ as the intersection. We will sometimes describe a square of affine schemes as a Schlessinger square if its opposite square is a Schlessinger square.

The point of this definition is that the squares we considered earlier are completely equivalent to Schlessinger squares:

Lemma 2.15. Suppose we have $p^{\prime}: A \rightarrow A^{\prime}$ and $p^{\prime \prime}: A \rightarrow A^{\prime \prime}$ defining a Schlessinger square. Then we have that

$$
A \cong A^{\prime} \times_{A^{\prime} \otimes_{A} A^{\prime \prime}} A^{\prime \prime}
$$

and $A^{\prime \prime} \rightarrow A^{\prime} \otimes_{A} A^{\prime \prime}$ is surjective.

Conversely, given $q^{\prime}: B^{\prime} \rightarrow B, q^{\prime \prime}: B^{\prime \prime} \rightarrow B$, with $q^{\prime \prime}$ surjective, then we have that

$$
B \cong B^{\prime} \otimes_{B^{\prime} \times{ }_{B} B^{\prime \prime}} B^{\prime \prime},
$$

and furthermore the maps $B^{\prime} \times_{B} B^{\prime \prime} \rightarrow B^{\prime}$ and $B^{\prime} \times_{B} B^{\prime \prime} \rightarrow B^{\prime \prime}$ define a Schlessinger square.

Proof. For both assertions, the key point is that given ring homomorphisms $p^{\prime}$ : $C \rightarrow C^{\prime}$ and $p^{\prime \prime}: C \rightarrow C^{\prime \prime}$, with $p^{\prime}$ surjective, we can easily check from the universal property of the tensor product that the natural map $C^{\prime \prime} \rightarrow C^{\prime} \otimes_{C} C^{\prime \prime}$, which factors through $C^{\prime \prime} /\left(p^{\prime \prime}\left(\operatorname{ker} p^{\prime}\right)\right)$, in fact induces an isomorphism $C^{\prime \prime} /\left(p^{\prime \prime}\left(\operatorname{ker} p^{\prime}\right)\right) \stackrel{\sim}{\rightarrow}$ $C^{\prime} \otimes_{C} C^{\prime \prime}$.

This lemma should not be surprising, as cofibered product of schemes corresponds to union in many Grothendieck (pre)topologies. The second part of the lemma says that the surjectivity condition imposed by Schlessinger implies that his $A$, which is in principal arbitrary, is in fact the (co)intersection of $A^{\prime}$ and $A^{\prime \prime}$ over $A^{\prime} \times{ }_{A} A^{\prime \prime}$.

We therefore have a definition of deformation stack which looks much closer to imposing a stack condition with respect to a collection of covers. Although the covers considered look quite different from the topologies normally considered in moduli theory, they are similar to those considered by Voevodsky for the "proper cdh-structure" in [22], so it is natural to ask whether our definition of deformation stack is equivalent to a stack condition for some Grothendieck (pre)topology on $\operatorname{Art}(\Lambda, k)$. We first review what it means to have a stack condition with respect to a given cover on a category fibered in groupoids.

Given a category $\mathcal{S}$ fibered in groupoids over a category $C$, and an arbitrary family of morphisms $\left\{U_{i} \rightarrow T\right\}$ in $C$, we have restriction maps on morphisms and objects of $\mathcal{S}$.

Indeed, given $\eta, \eta^{\prime} \in \mathcal{S}_{T}$, and a morphism $\varphi: \eta \rightarrow \eta^{\prime}$ over $\mathrm{id}_{T}$, we obtain via pullbacks morphisms $\varphi_{i}:\left.\left.\eta\right|_{U_{i}} \rightarrow \eta^{\prime}\right|_{U_{i}}$, and (iii) of Proposition 2.6 implies that if we pull back $\varphi_{i}$ and $\varphi_{j}$ to $U_{i, j}:=U_{i} \times_{T} U_{j}$, we have equality. That is, we have a map:

$$
\operatorname{Mor}_{\mathrm{id}_{T}}\left(\eta, \eta^{\prime}\right) \rightarrow\left\{\left\{\varphi_{i} \in \operatorname{Mor}_{\mathrm{id} U_{i}}\left(\left.\eta\right|_{U_{i}},\left.\eta^{\prime}\right|_{U_{i}}\right)\right\}_{i}:\left.\varphi_{i}\right|_{U_{i, j}}=\left.\varphi_{j}\right|_{U_{i, j}} \forall i, j\right\} .
$$


Similarly, given an object $\eta \in \mathcal{S}_{T}$, we obtain pullback objects $\left.\eta\right|_{U_{i}}$, and (i) and (ii) of Proposition 2.6 imply that for any $i, j$ both $\left.\left(\left.\eta\right|_{U_{i}}\right)\right|_{U_{i, j}}$ and $\left.\left(\left.\eta\right|_{U_{j}}\right)\right|_{U_{i, j}}$ are pullbacks $\left.\eta\right|_{U_{i, j}}$, and hence related by a unique isomorphism $\varphi_{i, j}$, which must then satisfy the cocycle condition for any $i, j, \ell$ after pullback to $U_{i, j, \ell}:=U_{i} \times_{T} U_{j} \times_{T}$ $U_{\ell}$. If, as is often done, we were to fix choices of pullbacks for every object, we would obtain a map:

$$
\operatorname{Obj}_{T} \rightarrow\left\{\begin{array}{r}
\left(\left\{\eta_{i} \in \operatorname{Obj}_{U_{i}}\right\}_{i},\left\{\varphi_{i, j} \in \operatorname{Mor}_{i_{T}}\left(\left.\eta_{i}\right|_{U_{i, j}},\left.\eta_{j}\right|_{U_{i, j}}\right)\right\}_{i, j}\right): \\
\left.\left.\varphi_{j, \ell}\right|_{U_{i, j, \ell}} \circ \varphi_{i, j}\right|_{U_{i, j, \ell}}=\left.\varphi_{i, \ell}\right|_{U_{i, j, \ell}} \forall i, j, \ell
\end{array}\right\} .
$$

A stack condition on $\mathcal{S}$ relative to $\left\{U_{i} \rightarrow T\right\}$ is, roughly speaking, a bijectivity condition on these two maps. More precisely, we have the following definition.

Definition 2.16. Given a category $\mathcal{S}$ fibered in groupoids over a category $C$, and an arbitrary family of morphisms $\left\{U_{i} \rightarrow T\right\}$ in $C$, we say that $\mathcal{S}$ satisfies the stack condition relative to $\left\{U_{i} \rightarrow T\right\}$ if the following holds:

(i) (Morphisms form a sheaf) The map (2.2) is always a bijection.

(ii) (Objects have effective descent) The map (2.3) is always surjective up to isomorphism on the right, where isomorphisms are required to commute with the $\varphi_{i, j}$.

Remark 2.17. Even in this more general context, it is easy to check that given condition (i), the map (2.3) is automatically injective if one mods out on the left by isomorphisms and on the right by isomorphisms commuting with the $\varphi_{i, j}$. Thus, assuming condition (i) we have that condition (ii) is equivalent to a bijectivity statement for (2.3) after modding out by the appropriate isomorphisms.

In order to examine whether our deformation stacks are in fact stacks for some Grothendieck (pre)topology, we are forced to consider two questions: first, is the condition we impose on the covers coming from Schlessinger squares equivalent to the stack condition; and second, is imposing the stack condition relative to those covers equivalent to imposing the stack condition relative to some Grothendieck (pre)topology?

We address the first question first. Looking at the definitions, we see that if we are given a Schlessinger square, it appears that we are imposing a stronger descent condition in the definition of deformation stack, since, considering for example the condition on objects, we require that a pair of objects on $A^{\prime}$ and $A^{\prime \prime}$ descend as long as they are isomorphic over $A^{\prime} \otimes_{A} A^{\prime \prime}$, with no condition on isomorphisms on the self-products $A^{\prime} \otimes_{A} A^{\prime}$ and $A^{\prime \prime} \otimes_{A} A^{\prime \prime}$ satisfying the cocycle condition. Although technical, this is a serious issue: for instance, in the étale topology it is crucial to use the weaker descent condition, as the stronger one will never be satisfied.

In the Zariski topology, this is not a problem, since every covering family $\left\{U_{i} \rightarrow T\right\}$ consists only of immersions, so $U_{i} \times_{T} U_{i}=U_{i}$. Similarly, in our situation the hypothesis that $A \rightarrow A^{\prime}$ is surjective means that $A^{\prime} \otimes_{A} A^{\prime}=A^{\prime}$, so the only difficulty arises in considering what happens on $A^{\prime \prime} \otimes_{A} A^{\prime \prime}$. We address this with the following lemma, which closely follows Voevodsky's theory of regular cd-structures, introduced in [21]. 
Lemma 2.18. Let $A \rightarrow A^{\prime}, A \rightarrow A^{\prime \prime}$ be two morphisms in $\operatorname{Art}(\Lambda, k)$, defining a Schlessinger square, and set $A^{\prime \prime \prime}=A^{\prime} \otimes_{A} A^{\prime \prime}$. Let $I=\operatorname{ker}\left(A^{\prime \prime} \otimes_{A} A^{\prime \prime} \rightarrow\right.$ $\left.A^{\prime \prime \prime} \otimes_{A^{\prime}} A^{\prime \prime \prime}\right)$, and let $\delta=\operatorname{ker}\left(A^{\prime \prime} \otimes_{A} A^{\prime \prime} \rightarrow A^{\prime \prime}\right)$.

Then we have $I \cap \delta=0$, so that the maps

$$
A^{\prime \prime} \otimes_{A} A^{\prime \prime} \rightarrow\left(A^{\prime \prime} \otimes_{A} A^{\prime \prime}\right) / I \cong A^{\prime \prime \prime} \otimes_{A^{\prime}} A^{\prime \prime \prime}
$$

and

$$
A^{\prime \prime} \otimes_{A} A^{\prime \prime} \rightarrow\left(A^{\prime \prime} \otimes_{A} A^{\prime \prime}\right) / \delta \cong A^{\prime \prime}
$$

define a Schlessinger square.

Proof. We first observe that both maps

$$
A^{\prime \prime} \otimes_{A} A^{\prime \prime} \rightarrow\left(A^{\prime \prime} \otimes_{A} A^{\prime \prime}\right) / I \text { and } A \rightarrow\left(A^{\prime \prime} \otimes_{A} A^{\prime \prime}\right) / \delta
$$

are surjective, so they define a Schlessinger square if and only if

$$
A^{\prime \prime} \otimes_{A} A^{\prime \prime} \rightarrow\left(A^{\prime \prime} \otimes_{A} A^{\prime \prime}\right) / I \times\left(A^{\prime \prime} \otimes_{A} A^{\prime \prime}\right) / \delta
$$

is injective, which is equivalent to the assertion that $I \cap \delta=0$.

We now claim that any element of $I$ is of the form $\sum_{i} z_{i} w_{i}$, with $w_{i} \in A^{\prime \prime} \otimes_{A}$ $A^{\prime \prime}$, and $z_{i} \in \operatorname{ker}\left(A \rightarrow A^{\prime}\right)$. Indeed, we have $A^{\prime \prime \prime} \otimes_{A^{\prime}} A^{\prime \prime \prime}=\left(A^{\prime} \otimes_{A} A^{\prime \prime}\right) \otimes_{A^{\prime}}$ $\left(A^{\prime} \otimes_{A} A^{\prime \prime}\right)=A^{\prime} \otimes_{A}\left(A^{\prime \prime} \otimes_{A} A^{\prime \prime}\right)$. Since $A \rightarrow A^{\prime}$ is surjective, $I$ is simply the ideal generated by $\operatorname{ker}\left(A \rightarrow A^{\prime}\right)$, as claimed.

Now let $w=\sum_{i} z_{i} w_{i}$ be as above; we want to show that if $w \in \delta$, then we must have $w=0$. Expanding terms as necessary, write $w_{i}=w_{i}^{\prime} \otimes w_{i}^{\prime \prime}$ for each $i$. Since $z_{i} \in \operatorname{ker}\left(A \rightarrow A^{\prime}\right)$, by the conditions on a Schlessinger square we have that $z_{i} w_{i}^{\prime}$ is the image of some $u_{i} \in A$, so we have $w=\sum_{i} 1 \otimes u_{i} w_{i}^{\prime \prime}=1 \otimes \sum_{i} u_{i} w_{i}^{\prime \prime}$. Now, if $w \in \delta$ we have $\sum_{i} u_{i} w_{i}^{\prime \prime}=0$, so it follows that $w=0$. Thus $I \cap \delta=0$, as desired.

Corollary 2.19. A category $\mathcal{S}$ fibered in groupoids over $\operatorname{Art}(\Lambda, k)$ is a deformation stack if and only if $\mathcal{S}_{k}$ is trivial, and $\mathcal{S}$ satisfies the stack condition for every cover associated to a Schlessinger square.

Proof. It is easy to check from the definitions and Lemma 2.15 that if $\mathcal{S}$ is a deformation stack, then it satisfies a stack condition for every Schlessinger square. Thus, we need only prove the converse.

We want to see that for every Schlessinger square

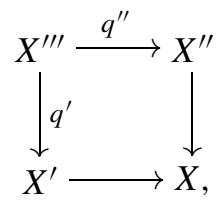


and every pair of objects $\eta, \eta^{\prime} \in \mathcal{S}_{X}$, a pair of morphisms $\varphi^{\prime} \in \operatorname{Mor}\left(\left.\eta\right|_{X^{\prime}},\left.\eta^{\prime}\right|_{X^{\prime}}\right)$ and $\varphi^{\prime \prime} \in \operatorname{Mor}\left(\left.\eta\right|_{X^{\prime \prime}},\left.\eta^{\prime}\right|_{X^{\prime \prime}}\right)$ which agree on $X^{\prime \prime \prime}$ automatically agree also on $X^{\prime} \times_{X} X^{\prime}$ and $X^{\prime \prime} \times_{X} X^{\prime \prime}$, and similarly for objects.

Suppose we have such morphisms $\varphi^{\prime}$ and $\varphi^{\prime \prime}$. Agreement on $X^{\prime} \times_{X} X^{\prime}$ is trivial; since $A \rightarrow A^{\prime}$ is assumed surjective, we have that $X^{\prime} \times{ }_{X} X^{\prime}=X^{\prime}$, with both projections being the identity map. For $X^{\prime \prime} \times_{X} X^{\prime \prime}$, we invoke the lemma, finding that we have another Schlessinger square defined by the maps

$$
X^{\prime \prime} \stackrel{\Delta}{\rightarrow} X^{\prime \prime} \times_{X} X^{\prime \prime} \text { and } X^{\prime \prime \prime} \times_{X^{\prime}} X^{\prime \prime \prime} \rightarrow X^{\prime \prime} \times_{X} X^{\prime \prime},
$$

where $\Delta$ denotes the diagonal map. We wish to show that $p_{1}^{*}\left(\varphi^{\prime \prime}\right)$ and $p_{2}^{*}\left(\varphi^{\prime \prime}\right)$ agree on $X^{\prime \prime} \times_{X} X^{\prime \prime}$. They certainly agree on $X^{\prime \prime}$. On the other hand, on $X^{\prime \prime \prime} \times_{X^{\prime}} X^{\prime \prime \prime}$ they give $\left(q^{\prime \prime} \times q^{\prime \prime}\right)^{*} p_{1}^{*}\left(\varphi^{\prime \prime}\right)$ and $\left(q^{\prime \prime} \times q^{\prime \prime}\right)^{*} p_{2}^{*}\left(\varphi^{\prime \prime}\right)$, which are the same as $p_{i}^{*}\left(q^{\prime \prime}\right)^{*}\left(\varphi^{\prime \prime}\right)$ for $i=1,2$. Since we assumed that $\varphi^{\prime}, \varphi^{\prime \prime}$ agreed on $X^{\prime \prime \prime}$, these give $p_{i}^{*}\left(q^{\prime}\right)^{*}\left(\varphi^{\prime}\right)$ for $i=1,2$, which are equal since the product is over $X^{\prime}$. We thus conclude that $p_{1}^{*}\left(\varphi^{\prime \prime}\right)=p_{2}^{*}\left(\varphi^{\prime \prime}\right)$ after restriction to either $X^{\prime \prime}$ or $X^{\prime \prime \prime} \times_{X^{\prime}} X^{\prime \prime \prime}$, so the stack condition implies agreement on $X^{\prime \prime} \times_{X} X^{\prime \prime}$, as desired.

The situation is similar for objects: suppose we are given $\eta^{\prime} \in \mathcal{S}_{X^{\prime}}$, and $\eta^{\prime \prime} \in$ $\mathcal{S}_{X^{\prime \prime}}$, together with an isomorphism $\varphi \in \operatorname{Mor}\left(\left.\eta^{\prime}\right|_{X^{\prime \prime \prime}},\left.\eta^{\prime \prime}\right|_{X^{\prime \prime \prime}}\right)$. We want to produce isomorphisms $\varphi^{\prime} \in \operatorname{Mor}_{X^{\prime} \times_{X} X^{\prime}}\left(p_{1}^{*} \eta^{\prime}, p_{2}^{*} \eta^{\prime}\right)$ and $\varphi^{\prime \prime} \in \operatorname{Mor}_{X^{\prime \prime} \times_{X} X^{\prime \prime}}\left(p_{1}^{*} \eta^{\prime \prime}, p_{2}^{*} \eta^{\prime \prime}\right)$ satisfying the cocycle condition, which in our case means that $\varphi^{\prime} \circ \varphi^{\prime}=\varphi^{\prime}, \varphi^{\prime \prime} \circ \varphi^{\prime \prime}=$ $\varphi^{\prime \prime}, \varphi \circ \varphi^{\prime}=\varphi$, and $\varphi^{\prime \prime} \circ \varphi=\varphi$, all interpreted suitably on the triple products. As before, there is nothing to do on $X^{\prime}$, since the products are canonically isomorphic to $X^{\prime}$ itself, and we can take $\varphi^{\prime}$ to be the identity. Also as before, we construct $\varphi^{\prime \prime}$ using the Schlessinger square provided by the lemma, and using the fact that we already established the desired statement for morphisms. Here we take $\varphi^{\prime \prime}$ to restrict to the identity on $X^{\prime \prime}$ and to $p_{2}^{*} \varphi \circ p_{1}^{*} \varphi^{-1}$ on $X^{\prime \prime \prime} \times_{X^{\prime}} X^{\prime \prime \prime}$. We then have to check that $\varphi^{\prime \prime} \circ \varphi^{\prime \prime}=\varphi^{\prime \prime}$ on $X^{\prime \prime} \times_{X} X^{\prime \prime} \times_{X} X^{\prime \prime}$. One first uses the same argument as in the above lemma to see that $X^{\prime \prime \prime} \times_{X^{\prime}} X^{\prime \prime \prime} \times_{X^{\prime}} X^{\prime \prime \prime} \rightarrow X^{\prime \prime} \times_{X} X^{\prime \prime} \times_{X} X^{\prime \prime}$, and $X^{\prime \prime} \times_{X} X^{\prime \prime} \stackrel{\text { id } \times \Delta}{\rightarrow} X^{\prime \prime} \times_{X} X^{\prime \prime} \times_{X} X^{\prime \prime}$ together form a Schlessinger square, and it then suffices to check the identity of morphisms after restriction to these, which is straightforward.

Finally, the identity $\varphi^{\prime \prime} \circ \varphi=\varphi$ on $X^{\prime} \times_{X} X^{\prime \prime} \times_{X} X^{\prime \prime}$ is easily checked via the observation that $X^{\prime} \times_{X} X^{\prime \prime} \times_{X} X^{\prime \prime}=X^{\prime \prime \prime} \times_{X^{\prime}} X^{\prime \prime \prime}$.

Thus, if $\mathcal{S}$ satisfies the condition for every cover arising from a Schlessinger square, it is a deformation stack, completing the proof of the corollary.

We see we have answered the first question positively, expressing deformation stacks in terms of a descent condition relative to a certain collection of covers. However, it turns out that the second question, on the relationship between Schlessinger squares and Grothendieck topologies, is less straightforward. The obvious Grothendieck (pre)topology to consider is the one generated by covers coming from Schlessinger squares. The problem with this arises from the condition that covers be stable under pullbacks, because it turns out that Schlessinger squares are not stable under pullback due to the condition on scheme-theoretic surjectivity. 
The following example of Schlessinger squares failing to be preserved under pushforward is based on a suggestion of Eisenbud.

Example 2.20. We let $A=k[x, t] /\left(x^{2}, t^{2}, x t\right), A^{\prime}=k$, and $A^{\prime \prime}=k[y, t] /\left(y^{2}, t^{2}\right)$. The map $A \rightarrow A^{\prime}$ is the obvious quotient map, while the map $A \rightarrow A^{\prime \prime}$ is defined by sending $t$ to itself and $x$ to $y t$. We note that $A \rightarrow A^{\prime}$ is surjective and $A \rightarrow A^{\prime \prime}$ is injective, and that $\operatorname{ker}\left(A \rightarrow A^{\prime}\right)=(x, t)$ maps to the ideal $(t) \subseteq A^{\prime \prime}$. Thus, we obtain a Schlessinger square.

Now let $B=k[x] /\left(x^{2}\right)$. We take the map $A \rightarrow B$ obtained by sending $t$ to 0 . If we push forward our square along $A \rightarrow B$, we obtain $B^{\prime}=k, B^{\prime \prime}=k[y] /\left(y^{2}\right)$, and the map $B \rightarrow B^{\prime \prime}$ sends $x$ to 0 . Therefore the condition $B \hookrightarrow B^{\prime} \times B^{\prime \prime}$ fails, and the pushforward is not a Schlessinger square.

We see from this example that we should not expect any deformation problem to satisfy a stack condition for the cover associated to this pushforward square: indeed, if the problem were prorepresentable, its tangent space would be automatically forced to be 0 by such a condition. Thus, we see that it is not reasonable to hope to impose a stack condition for the Grothendieck (pre)topology generated by Schlessinger squares, and in particular such a condition is far stronger than the condition of being a deformation stack.

The previous example might appear somewhat contrived, so one might naturally ask whether there is a "good" class of Schlessinger squares, stable under pullback, and which are nonetheless sufficient for our purposes. However, we find that it is simple to modify the previous example to apply to even the most basic Schlessinger squares.

Example 2.21. Let $A=k[x, y] /\left(x^{2}, x y, y^{2}\right)$, equipped with the canonical quotient maps to $A^{\prime}=k[x] /\left(x^{2}\right)$ and $A^{\prime \prime}=k[y] /\left(y^{2}\right)$. This is one of the most fundamental Schlessinger squares, obtained from the projection maps of $k[\epsilon] \times{ }_{k} k[\epsilon]$. Let $B=k[x, t] /\left(x^{2}, t^{2}\right)$, and take the map $A \rightarrow B$ which sends $x \mapsto x$ and $y \mapsto x t$. Pushing forward our Schlessinger square as before, we check that scheme-theoretic surjectivity once again fails to be preserved.

These examples strongly suggest that the theory of deformation stacks does in fact lie outside the usual framework of Grothendieck topologies.

\subsection{Examples}

We discuss several examples of deformation problems which naturally constitute deformation stacks.

Example 2.22. Deformations of sheaves. Given a scheme $X_{\Lambda}$ over $\operatorname{Spec} \Lambda$, let $X:=\left.X_{\Lambda}\right|_{\text {Spec } k}$, and suppose we have $\mathcal{E}$, a quasicoherent $\mathcal{O}_{X}$-module. We consider the deformation problem classifying flat deformations of $\mathcal{E}$ over $\left.X_{\Lambda}\right|_{A}$ for different $A \in \operatorname{Art}(\Lambda, k)$. Our deformation stack $\mathcal{D} e f_{X_{\Lambda}}(\mathcal{E})$ as a category has objects

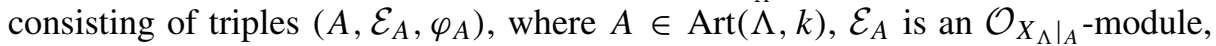
flat over $A$, and $\varphi_{A}: \mathcal{E}_{A} \rightarrow \mathcal{E}$ a morphism of $\mathcal{O}_{X_{A}}$-modules such that the map 
$\left.\varphi_{A}\right|_{k}: \mathcal{E}_{A} \otimes_{A} k \rightarrow \mathcal{E}$ induced by $\varphi_{A}$ and $k \rightarrow \mathcal{E}$ is an isomorphism. Morphisms $\left(A^{\prime}, \mathcal{E}_{A^{\prime}}, \varphi_{A^{\prime}}\right) \rightarrow\left(A, \mathcal{E}_{A}, \varphi_{A}\right)$ consist of pairs $(f, \varphi)$, with $f: A \rightarrow A^{\prime}$, and $\varphi: \mathcal{E}_{A} \rightarrow \mathcal{E}_{A^{\prime}}$ a homomorphism of $\mathcal{O}_{X_{A}}$-modules such that $\varphi_{A^{\prime}} \circ \varphi=\varphi_{A}$. The functor to $\operatorname{Art}(\Lambda, k)$ is then the forgetful one.

It is clear that $\mathcal{D} e f\left(X_{\Lambda}, \mathcal{E}\right)$ is a category fibered in groupoids over $\operatorname{Art}(\Lambda, k)$, but the fact that it is a deformation stack (like the fact that the associated functor satisfies Schlessinger's (H1) and (H2)) requires some justification. However, we see that essentially the same argument will work. Indeed, if we fix $A^{\prime} \rightarrow A, A^{\prime \prime} \rightarrow A$, and write $B=A^{\prime} \times_{A} A^{\prime \prime}$, we need to check two facts. First, if we are given any objects $\left(B, \mathcal{E}_{B}, \varphi_{B}\right),\left(B, \mathcal{E}_{B}^{\prime}, \varphi_{B}^{\prime}\right)$, we abbreviate them by $\mathcal{E}_{B}$ and $\mathcal{E}_{B}^{\prime}$, and their restrictions to $A, A^{\prime}, A^{\prime \prime}$ similarly. We then want that

$$
\operatorname{Mor}_{X_{B}}\left(\mathcal{E}_{B}, \mathcal{E}_{B}^{\prime}\right)=\operatorname{Mor}_{X_{A^{\prime}}}\left(\mathcal{E}_{A^{\prime}}, \mathcal{E}_{A^{\prime}}^{\prime}\right) \times_{\operatorname{Mor}_{X_{A}}\left(\mathcal{E}_{A}, \mathcal{E}_{A}^{\prime}\right)} \operatorname{Mor}_{X_{A^{\prime \prime}}}\left(\mathcal{E}_{A^{\prime \prime}}, \mathcal{E}_{A^{\prime \prime}}^{\prime}\right) \text {. }
$$

It is enough to work locally, and the desired identity for morphisms of modules over $B, A^{\prime}, A^{\prime \prime}, A$ follows trivially from the fact that the modules in question are free, by Corollary A.2. It then remains to check that a morphism of modules over $B$ is in fact a morphism over $\mathcal{O}_{X_{B}}$ if and only if the restrictions are morphisms over $\mathcal{O}_{X_{A^{\prime}}}$ and $\mathcal{O}_{X_{A^{\prime \prime}}}$, which similarly follows because equality of elements of a free $B$-module may be checked after restriction to $A^{\prime}$ and $A^{\prime \prime}$.

Second, if we are given objects $\left(A^{\prime}, \mathcal{E}_{A^{\prime}}, \varphi_{A^{\prime}}\right)$ and $\left(A^{\prime \prime}, \mathcal{E}_{A^{\prime \prime}}, \varphi_{A^{\prime \prime}}\right)$, together with an isomorphism of their restrictions to $A$, we need to have an object $\left(B, \mathcal{E}_{B}, \varphi_{B}\right)$ inducing the given objects after pullback to $A^{\prime}$ and $A^{\prime \prime}$, with the given isomorphism after pullback to $A$. Set $\mathcal{E}_{B}=\mathcal{E}_{A^{\prime}} \times \mathcal{E}_{A} \mathcal{E}_{A^{\prime \prime}}$. To check the desired flatness and isomorphisms, it is enough to work locally on $X$, and the desired statements then follow from Lemma A.3 below. Using the natural map $\mathcal{O}_{X_{B}} \rightarrow \mathcal{O}_{X_{A^{\prime}}} \times_{\mathcal{O}_{X_{A}}} \mathcal{O}_{X_{A^{\prime \prime}}}$, we induce an $\mathcal{O}_{X_{B}}$-module structure on $\mathcal{E}_{B}$, and quasicoherence can then be checked from the fact that module fiber product commutes with localization, so we obtain the desired descent condition.

Example 2.23. Deformations of schemes. Given a scheme $X$ over Spec $k$, we consider the deformation problem classifying flat deformations of $X$ over Spec $A$ for different $A \in \operatorname{Art}(\Lambda, k)$. Our deformation stack $\mathcal{D} e f(X)$ has objects $\left(A, X_{A}, \varphi_{A}\right)$, where $A \in \operatorname{Art}(\Lambda, k), X_{A}$ is a scheme flat over $A$, and $\varphi_{A}: X \rightarrow X_{A}$ is a morphism over the closed imbedding Spec $k \rightarrow$ Spec $A$, inducing an isomorphism after restriction to Spec $k$. Morphisms $\left(A^{\prime}, X_{A^{\prime}}, \varphi_{A^{\prime}}\right) \rightarrow\left(A, X_{A}, \varphi_{A}\right)$ consist of $(f, \varphi)$, with $f: A \rightarrow A^{\prime}$, and $\varphi: X_{A^{\prime}} \rightarrow X_{A}$ a morphism over $f$, inducing an isomorphism after restriction to $\operatorname{Spec} A^{\prime}$, and with $\varphi_{A}=\varphi \circ \varphi_{A^{\prime}}$.

The proof that this gives a deformation stack is similar to the case of sheaves. Indeed, the construction proceeds in the same fashion, except that one has to work with algebras and morphisms of algebras, and it is also necessary to check that the fiber product construction of the sheaf case yields a scheme when applied to $\mathcal{O}_{X_{A}}, \mathcal{O}_{X_{A^{\prime}}}, \mathcal{O}_{X_{A^{\prime \prime}}}$. As with checking quasicoherence above, this is simply a matter of fiber product of rings commuting with localization.

Example 2.24. Deformations of quotient sheaves. Given a scheme $X_{\Lambda}$ over $\operatorname{Spec} \Lambda$, and a quasicoherent $\mathcal{O}_{X_{\Lambda}}$-module $\mathcal{E}_{\Lambda}$, set $X=\left.X_{\Lambda}\right|_{k}$ and $\mathcal{E}:=\left.\mathcal{E}_{\Lambda}\right|_{k}$. If we have 
$\mathcal{E} \rightarrow \mathcal{F}$ a quasicoherent quotient module of $\mathcal{E}$, we have the deformation problem classifying deformations of $\mathcal{F}$ as a quotient of $\left.\mathcal{E}_{\Lambda}\right|_{A}$ for $A \in \operatorname{Art}(\Lambda, k)$. Our deformation stack $\mathcal{D} e f_{X_{\Lambda}, \mathcal{E}_{\Lambda}}(\mathcal{F})$ has objects $\left(A, \mathcal{F}_{A}, \varphi_{A}\right)$, with $\mathcal{F}_{A}$ flat over $A$, and a surjective map $\varphi_{A}:\left.\mathcal{E}_{\Lambda}\right|_{A} \rightarrow \mathcal{F}_{A}$ inducing an isomorphism $\mathcal{F}_{A} \otimes_{A}$ Spec $k \stackrel{\sim}{\rightarrow} \mathcal{F}$. Morphisms $\left(A^{\prime}, \mathcal{F}_{A^{\prime}}, \varphi_{A^{\prime}}\right) \rightarrow\left(A, \mathcal{F}_{A}, \varphi_{A}\right)$ consist of $f: A \rightarrow A^{\prime}$ such that the induced map $\left(\mathcal{E}_{\Lambda} \otimes_{\Lambda} A\right) \otimes_{A} A^{\prime} \rightarrow \mathcal{E}_{\Lambda} \otimes_{\Lambda} A^{\prime}$ yields an isomorphism $\mathcal{F}_{A} \otimes_{A} A^{\prime} \stackrel{\sim}{\rightarrow} \mathcal{F}_{A^{\prime}}$.

Because there is at most one morphism lying over a given morphism of $\operatorname{Art}(\Lambda, k)$, we need only check that given $\left(A^{\prime}, \mathcal{F}_{A^{\prime}}, \varphi_{A^{\prime}}\right)$ and $\left(A^{\prime \prime}, \mathcal{F}_{A^{\prime \prime}}, \varphi_{A^{\prime \prime}}\right)$ both restricting to some $\left(A, \mathcal{F}_{A}, \varphi_{A}\right)$, there exists a unique $\left(B, \mathcal{F}_{B}, \varphi_{B}\right)$ restricting to the given objects over $A^{\prime}$ and $A^{\prime \prime}$. But by flatness and the argument of Example 2.22, we can take $\mathcal{F}_{B}=\mathcal{F}_{A^{\prime}} \times \mathcal{F}_{A} \mathcal{F}_{A^{\prime \prime}}$, and $\varphi_{B}$ induced by the natural map (which, despite the lack of flatness hypothesis on $\mathcal{E}$, one checks is surjective) $\mathcal{E}_{B} \rightarrow \mathcal{E}_{A^{\prime}} \times \mathcal{E}_{A} \mathcal{E}_{A^{\prime \prime}}$. It is then easy to check that the construction of $\left(B, \mathcal{F}_{B}, \varphi_{B}\right)$ provides an inverse to the natural map from quotients over $B$ to pairs of quotients over $A^{\prime}$ and $A^{\prime \prime}$ agreeing on $A$, so we obtain the desired assertion.

Example 2.25. Deformations of subschemes. Given a scheme $X_{\Lambda}$ over $\operatorname{Spec} \Lambda$, set $X=\left.X_{\Lambda}\right|_{\text {Spec } k}$. Given $Z \subseteq X$ a closed subscheme, we have the deformation problem classifying deformations of $Z$ as a subscheme of $X$. The deformation stack De $f_{X_{\Lambda}}(Z)$ has objects $\left(A, Z_{A}\right)$, with $A \in \operatorname{Art}(\Lambda, k)$, and $Z_{A}$ a closed subscheme of $\left.X_{\Lambda}\right|_{A}$, flat over $A$, and such that $\left.Z_{A}\right|_{\text {Spec } k}=Z$. Morphisms $\left(A^{\prime}, Z_{A^{\prime}}\right) \rightarrow\left(A, Z_{A}\right)$ consist of $f: A \rightarrow A^{\prime}$ such that $\left.Z_{A^{\prime}}\right|_{A}$ maps isomorphically to $Z_{A}$ under the natural $\left.\left.\operatorname{map}\left(\left.X_{\Lambda}\right|_{A^{\prime}}\right)\right|_{A} \rightarrow X_{\Lambda}\right|_{A}$.

In fact, this is a special case of deformations of quotient sheaves, obtained by setting $\mathcal{E}_{\Lambda}=\mathcal{O}_{X_{\Lambda}}$ and $\mathcal{F}=\mathcal{O}_{Z}$; the kernel of any map of $\mathcal{O}_{X_{A}}$-modules $\mathcal{O}_{X_{A}} \rightarrow \mathcal{O}_{Z_{A}}$ is necessarily an ideal, so $\mathcal{O}_{Z_{A}}$ inherits a unique algebra structure, and the flatness of $Z$ over $A$ is equivalent to our condition for quotient sheaves.

Example 2.26. Deformations of morphisms. Given a pair of schemes $X_{\Lambda}, Y_{\Lambda}$ locally of finite type over $\Lambda$, with $X_{\Lambda}$ flat and $Y_{\Lambda}$ separated, set $X=\left.X_{\Lambda}\right|_{\operatorname{Spec} k}, Y=$ $Y_{\Lambda} \mid$ Spec $k$. If we have also a morphism $f: X \rightarrow Y$, we have the deformation problem classifying deformations of $f$. The deformation stack $\mathcal{D} e f_{X_{\Lambda}, Y_{\Lambda}}(f)$ has objects $\left(A, f_{A}\right)$ with $A \in \operatorname{Art}(\Lambda, k)$, and $f_{A}:\left.\left.X_{\Lambda}\right|_{A} \rightarrow Y_{\Lambda}\right|_{A}$ such that $f_{A} \mid \operatorname{Spec} k=f$. Morphisms $\left(A^{\prime}, f_{A^{\prime}}\right) \rightarrow\left(A, f_{A}\right)$ consist of $g: A \rightarrow A^{\prime}$ such that $\left.f_{A}\right|_{A^{\prime}}=f_{A^{\prime}}$.

We claim that with our hypotheses on $X$ and $Y$, deformations of morphisms are a special case of deformations of closed subschemes, by considering the graph of the morphism. Because $Y$ is separated, the graph is a closed subscheme, and it remains to check that any deformation of a graph $\Gamma \subseteq X \times_{k} Y$ will still have $p_{1}$ : $\Gamma_{A} \rightarrow X_{A}$ an isomorphism, which follows from the flatness of $f$ by [6, Corollary 17.9.5]. Note also that the condition that $p_{1}$ be an isomorphism immediately implies that for any deformation of $f$, we have $\Gamma_{A}$ flat over $A$.

Example 2.27. Deformations of connections. Given a scheme $X_{\Lambda}$ smooth over $\Lambda$, and a quasicoherent $\mathcal{O}_{X_{\Lambda}}$-module $\mathcal{E}_{\Lambda}$ flat over $\Lambda$, write $X=\left.X_{\Lambda}\right|_{k}$ and $\mathcal{E}=\left.\mathcal{E}_{\Lambda}\right|_{k}$. Suppose we have a connection $\nabla$ on $\mathcal{E}$. We then have the deformation problem classifying deformations of $\nabla$. The deformation stack $\mathcal{D} e f_{X_{\Lambda}, \mathcal{E}_{\Lambda}}(\nabla)$ has objects 
consisting of $\left(A, \nabla_{A}\right)$, with $A \in \operatorname{Art}(\Lambda, k)$ and $\nabla_{A}$ a connection on $\mathcal{E}_{\Lambda} \otimes_{\Lambda} A$ such that $\left.\nabla_{A}\right|_{k}=\nabla$. Morphisms $\left(A^{\prime}, \nabla_{A^{\prime}}\right) \rightarrow\left(A, \nabla_{A}\right)$ consist of $f: A \rightarrow A^{\prime}$ such that $\left.\nabla_{A}\right|_{A^{\prime}}=\nabla_{A^{\prime}}$.

As in the case of quotient sheaves, there are no automorphisms, so we only need to check that any $\nabla_{A^{\prime}}$ and $\nabla_{A^{\prime \prime}}$ agreeing over $A$ are obtained from a unique connection $\nabla_{B}$ over $B:=A^{\prime} \times_{A} A^{\prime \prime}$. As in the case of deformations of sheaves, we have $\mathcal{E}_{B}=\mathcal{E}_{A^{\prime}} \times \mathcal{E}_{A} \mathcal{E}_{A^{\prime \prime}}$, and we also see by smoothness of $X_{\Lambda}$ that $\Omega_{X_{B} / B}^{1}=$

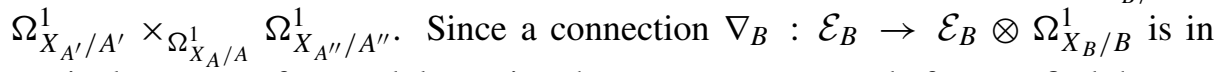
particular a map of $B$-modules, using the same argument as before we find that we get a unique map $\nabla_{B}$ of $B$-modules from $\nabla_{A^{\prime}}$ and $\nabla_{A^{\prime \prime}}$. Finally, checking that such a map is a connection if and only if $\nabla_{A^{\prime}}$ and $\nabla_{A^{\prime \prime}}$ are connections is the same as checking $\mathcal{O}_{X}$-linearity of morphisms in the case of deformations of sheaves.

We mention briefly a number of additional examples of deformation stacks, which will be studied in more detail in [16]. These involve combining previous examples: deformations of schemes together with morphisms to a fixed scheme, deformations of connections together with the underlying sheaf, and deformations of subsheaves together with the ambient sheaf.

Indeed, it seems that every deformation problem of interest can be given the structure of a deformation stack, and that furthermore, checking that a problem satisfies Schlessinger's (H1) and (H2) entails checking the axioms for a deformation stack. Thus, even though the conditions are technically more stringent than those of a deformation functor, in practice they seem neither more restrictive nor harder to check.

\subsection{Additive structure}

Throughout the following, we suppose that $\mathcal{S}$ is a deformation stack, and $f: A^{\prime} \rightarrow$ $A$ a small extension with kernel $I$. An important special case is when $V$ is a $k$ vector space, and we set $A^{\prime}=k[V], I=V$. In this case, we obtain an addition law on the category $\mathcal{S}_{k[V]}$ in a strong sense. In full generality, we have a certain additive structure which we describe and explore. We first make some preliminary definitions.

Notation 2.28. We denote by $T_{I}$ the set of isomorphism classes of $\mathcal{S}_{k[I]}$, and by $A_{I}$ the group $\operatorname{Aut}\left(\zeta_{I}\right)$ in $\mathcal{S}_{k[I]}$.

Thus, if $k[I]=k[\epsilon]$, then $T_{I}$ is the tangent space, and $A_{I}$ is the infinitesimal automorphism group of $\mathcal{S}$. The following notation deals with liftings of objects and morphisms over small extensions.

Notation 2.29. For a fixed $\eta \in \mathcal{S}_{A}$, we write

$$
T_{\eta, A^{\prime}}:=\left\{\left(\eta^{\prime}, \varphi\right): \eta^{\prime} \in \mathcal{S}_{A^{\prime}}, \varphi: \eta \rightarrow \eta^{\prime} \text { over } A^{\prime} \rightarrow A\right\} / \cong,
$$

where isomorphisms are applied simultaneously to $\eta^{\prime}$ and $\varphi$.

Similarly, fixing $\eta^{\prime} \in \mathcal{S}_{A^{\prime}}$ and $\varphi \in \operatorname{Aut}\left(\left.\eta^{\prime}\right|_{A}\right)$, we write

$$
A_{\varphi, \eta^{\prime}}:=\left\{\varphi^{\prime} \in \operatorname{Aut}\left(\eta^{\prime}\right):\left.\varphi^{\prime}\right|_{A}=\varphi\right\} .
$$


We define below a categorical addition law

$$
+_{A^{\prime}, I}: \mathcal{S}_{A^{\prime}} \times \mathcal{S}_{k[I]} \rightarrow \mathcal{S}_{A^{\prime}}
$$

an important consequence of which will be the following.

Corollary 2.30. The addition law $+_{k[I], I}$ gives canonical abelian group structures to $T_{I}$ and $A_{I}$, with the addition on the latter agreeing with composition. These addition laws are functorial under linear maps $I \rightarrow J$.

If we fix $\eta \in \mathcal{S}_{A}$, the addition law $+_{A^{\prime}, I}$ induces a canonical action of $T_{I}$ on $T_{\eta}, A^{\prime}$, making $T_{\eta, A^{\prime}}$ into a pseudotorsor for $T_{I}$.

Lastly, fixing $\eta^{\prime} \in \mathcal{S}_{A^{\prime}}$, the addition law ${ }_{A^{\prime}, I}$ induces a canonical group isomorphism $A_{I} \stackrel{\sim}{\rightarrow} A_{\mathrm{id}, \eta^{\prime}}$. Therefore for any $\varphi \in \operatorname{Aut}\left(\left.\eta^{\prime}\right|_{A}\right)$, we have a canonical action of $A_{I}$ on $A_{\varphi, \eta^{\prime}}$, making $A_{\varphi, \eta^{\prime}}$ a pseudotorsor for $A_{I}$.

Moreover, both pseudotorsor structures are functorial in the following sense: given another small extension $B^{\prime} \rightarrow B$ with kernel $J$, and a homomorphism $A^{\prime} \rightarrow$ $B^{\prime}$ which induces maps $I \rightarrow J$ and therefore $A \rightarrow B$, then the additive structures commute with the induced restriction maps.

Recall that we say that a set $S$ is a pseudotorsor over a group $G$ if $S$ is either empty or a torsor over $G$. This result can be seen as saying that if $\mathcal{S}$ is a deformation stack with an obstruction space, then we automatically obtain the additive part of a generalized tangent-obstruction theory in the sense of Fantechi and Göttsche; in fact, we will see later that we obtain the full theory; see Remark 2.42 below. However, we emphasize that our categorical additive structure does not merely yield the above corollary, but also streamlines checking compatibilities when constructing the obstruction maps in the proof Theorem 1.1.

The first step in defining $+_{A^{\prime}, I}$ is the following basic proposition, which essentially rephrases the deformation stack axioms in the form of operations on objects and morphisms. Indeed, this proposition is the only place in which we use the deformation stack axioms. Although we state the proposition quite generally, we will mainly be interested in the case $n=2$, with $\mathbf{A}=A^{\prime} \times_{k} k[I]$ or $k[I] \times_{k} k[I]$. We will however use other cases in the proofs of certain technical statements below.

Proposition 2.31. Fix $n \geq 2$, as well as $A_{1}^{\prime}, \ldots, A_{n}^{\prime}, A_{1}, \ldots, A_{n-1} \in \operatorname{Art}(\Lambda, k)$ with surjective maps $A_{i}^{\prime} \rightarrow A_{i}, A_{i+1}^{\prime} \rightarrow A_{i}$ for $i=1, \ldots, n-1$, and set

$$
\mathbf{A}=A_{1}^{\prime} \times_{A_{1}} A_{2}^{\prime} \times_{A_{2}} \cdots \times_{A_{n-1}} A_{n}^{\prime} .
$$

Given $\eta_{i}^{\prime} \in \mathcal{S}_{A_{i}^{\prime}}$ for $i=1, \ldots, n$ and $\eta_{i} \in \mathcal{S}_{A_{i}}$ for $i=1, \ldots, n-1$, with maps $\eta_{i} \rightarrow \eta_{i}^{\prime}$ and $\eta_{i} \rightarrow \eta_{i+1}^{\prime}$ lying over the given maps $A_{i}^{\prime} \rightarrow A_{i}$ and $A_{i+1}^{\prime} \rightarrow A_{i}$ respectively, there exists an object

$$
\eta_{1}^{\prime} *_{\eta_{1}} \cdots *_{\eta_{n-1}} \eta_{n}^{\prime} \in \mathcal{S}_{\mathbf{A}}
$$

having maps $q_{i}$ from $\eta_{i}^{\prime}$, which lie over the projection maps $p_{i}$, and commuting with the given maps from each $\eta_{i}$. Furthermore, the tuple $\left(\eta_{1}^{\prime} *_{\eta_{1}} \cdots *_{\eta_{n-1}} \eta_{n}^{\prime}, q_{1}, \ldots, q_{n}\right)$ is unique up to unique isomorphism. 
If we are given additionally $\mu_{i}^{\prime} \in \mathcal{S}_{A_{i}^{\prime}}$ with maps from the given $\eta_{i}$ as above, and morphisms $\varphi_{i}^{\prime}: \eta_{i}^{\prime} \rightarrow \mu_{i}^{\prime}$ in $\mathcal{S}_{A_{i}^{\prime}}$ commuting with the maps to the $\eta_{i}$, there is a unique morphism

$$
\varphi_{1}^{\prime} *_{\eta_{1}} \cdots *_{\eta_{n-1}} \varphi_{n}^{\prime}: \eta_{1}^{\prime} *_{\eta_{1}} \cdots *_{\eta_{n-1}} \eta_{n}^{\prime} \rightarrow \mu_{1}^{\prime} *_{\eta_{1}} \cdots *_{\eta_{n-1}} \mu_{n}^{\prime}
$$

in $\mathcal{S}_{\mathbf{A}}$ recovering the $\varphi_{i}^{\prime}$ under projection. The $*$ operation on morphisms commutes with composition. If the choice of $\eta_{1}^{\prime} *_{\eta_{1}} \cdots *_{\eta_{n-1}} \eta_{n}^{\prime}$ or $\mu_{1}^{\prime} *_{\eta_{1}} \cdots *_{\eta_{n-1}} \mu_{n}^{\prime}$ is changed, the resulting $\varphi_{1}^{\prime} *_{\eta_{1}} \cdots *_{\eta_{n-1}} \varphi_{n}^{\prime}$ changes by the corresponding unique isomorphism(s).

Finally, if for any $i$ we have $A_{i}=k$, then $\eta_{i}$ and the maps $\eta_{i} \rightarrow \eta_{i}^{\prime}$ and $\eta_{i} \rightarrow \eta_{i+1}^{\prime}$ are irrelevant, so we omit $\eta_{i}$ from our notation.

Proof. The existence of $\eta_{1}^{\prime} *_{\eta_{1}} \cdots *_{\eta_{n-1}} \eta_{n}^{\prime}$ together with the $q_{i}$ follows immediately from induction on condition (ii) of a deformation stack. The existence of a unique isomorphism between any two tuples then follows similarly from condition (i).

The existence and uniqueness of $\varphi_{1}^{\prime} *_{\eta_{1}} \cdots *_{\eta_{n-1}} \varphi_{n}^{\prime}$ similarly follows from condition (i) of the deformation stack axioms. The fact that $*$ commutes with composition then follows formally from the definition, as does the behavior under change of the choice of $\eta_{1}^{\prime} *_{\eta_{1}} \cdots *_{\eta_{n-1}} \eta_{n}^{\prime}$ or $\mu_{1}^{\prime} *_{\eta_{1}} \cdots *_{\eta_{n-1}} \mu_{n}^{\prime}$.

Finally, if $A_{i}=k$, because $\mathcal{S}_{k}$ is trivial any choice of $\eta_{i}$ is uniquely isomorphic to $\zeta_{0}$, and there is always a unique morphism from $\eta_{i}$, so the conditions on restricting to $\eta_{i}$ and commuting with the given maps to $\eta_{i}$ are always satisfied automatically, and we are justified in omitting $\eta_{i}$ from our notation.

From the existence and uniqueness of $\eta_{1} * \eta_{2}$, we can now define our additive structure via pushforward under the ring map

$$
\sigma_{A^{\prime}, I}: A^{\prime} \times_{k} k[I] \rightarrow A^{\prime}
$$

defined by $(x, \pi(x)+i) \mapsto x+y$.

Definition 2.32. An addition datum for $A^{\prime}$ is a choice, for all $\eta_{1} \in \mathcal{S}_{A^{\prime}}, \eta_{2} \in$ $\mathcal{S}_{k[I]}$, of objects $\eta_{1} * \eta_{2}$ and $\sigma_{*}\left(\eta_{1} * \eta_{2}\right)$, together with the associated morphisms.

Any addition datum for $A^{\prime}$ will yield an addition functor. We will also need an associativity isomorphism induced by the datum. This involves both $A^{\prime}$ and $k[I]$, so when $A^{\prime} \neq k[I]$, we have to choose an addition datum for both $A^{\prime}$ and $k[I]$ to express the isomorphism. In order to reduce notation, we will choose our addition functors to satisfy certain additional properties. We also phrase the following theorem in a slightly stronger form than is strictly necessary, so that we can conclude immediately that $\mathcal{S}_{k[I]}$ is a Picard category in the sense of Deligne (see [3, Definition 1.4.2 of Expose XVIII]). This will not be used here, but will be convenient in [16].

Theorem 2.33. Any choices of addition data for $A^{\prime}$ and (when $\left.A^{\prime} \neq k[I]\right)$ for $k[I]$ induce an additive structure consisting of functors

$$
+_{A^{\prime}, I}: \mathcal{S}_{A^{\prime}} \times \mathcal{S}_{k[I]} \rightarrow \mathcal{S}_{A^{\prime}}
$$


and

$$
+_{k[I], I}: \mathcal{S}_{k[I]} \times \mathcal{S}_{k[I]} \rightarrow \mathcal{S}_{k[I]}
$$

and an isomorphism of functors

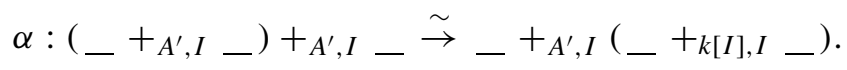

Furthermore, there exist addition data for $A^{\prime}$ and $k[I]$ such that:

(i) for any $\eta \in \mathcal{S}_{k[I]}$, the functor ${ }_{A^{\prime}, I} \eta: \mathcal{S}_{A^{\prime}} \rightarrow \mathcal{S}_{A^{\prime}}$ is an equivalence of categories;

(ii) $\zeta_{I} \in \mathcal{S}_{k[I]}$ is an identity object, in the sense that _ ${ }_{A^{\prime}, I} \zeta_{I}: \mathcal{S}_{A^{\prime}} \rightarrow \mathcal{S}_{A^{\prime}}$ is the identity functor;

(iii) $+_{k[I], I}$ is commutative, in the sense that $+_{k[I], I}=+_{k[I], I} \circ \mathrm{sw}$, where sw: $\mathcal{S}_{k[I]} \times \mathcal{S}_{k[I]} \rightarrow \mathcal{S}_{k[I]} \times \mathcal{S}_{k[I]}$ is the functor switching factors;

(iv) for any $\eta_{1}, \eta_{2}, \eta_{3}, \eta_{4} \in \mathcal{S}_{k[I]}$, we have the identities

$\left(\mathrm{id}_{\eta_{1}}+\alpha_{\eta_{2}, \eta_{3}, \eta_{4}}\right) \circ \alpha_{\eta_{1}, \eta_{2}+\eta_{3}, \eta_{4}} \circ\left(\alpha_{\eta_{1}, \eta_{2}, \eta_{3}}+\mathrm{id}_{\eta_{4}}\right)=\alpha_{\eta_{1}, \eta_{2}, \eta_{3}+\eta_{4}} \circ \alpha_{\eta_{1}+\eta_{2}, \eta_{3}, \eta_{4}}$

and

$$
\alpha_{\eta_{1}, \eta_{2}, \eta_{3}} \circ \alpha_{\eta_{3}, \eta_{1}, \eta_{2}}=\alpha_{\eta_{1}, \eta_{3}, \eta_{2}} .
$$

In the above and hereafter, we denote by $\alpha_{\eta_{1}, \eta_{2}, \eta_{3}}:\left(\eta_{1}+\eta_{2}\right)+\eta_{3} \stackrel{\sim}{\rightarrow} \eta_{1}+\left(\eta_{2}+\eta_{3}\right)$ the isomorphism obtained from $\alpha$.

The additive structures induced by different choices of addition data are related by unique isomorphism of functors. Addition is also functorial in the following sense: given a small extension $B^{\prime} \rightarrow B$ with kernel $J$ and a map $A^{\prime} \rightarrow B^{\prime}$ inducing $I \rightarrow J$ and therefore $A \rightarrow B$, if we have chosen $+_{A^{\prime}, I}$ and $+_{B^{\prime}, J}$ as above, then given $\eta_{1} \in \mathcal{S}_{A^{\prime}}$ and $\eta_{2} \in \mathcal{S}_{k[I]}$, and choices of pushforwards $\left.\eta_{1}\right|_{B^{\prime}}$ and $\left.\eta_{2}\right|_{k[J]}$, there is a canonical map $\left.\eta_{1}\right|_{B^{\prime}}+\left.\eta_{2}\right|_{k[J]} \rightarrow \eta_{1}+\eta_{2}$ lying over $A^{\prime} \rightarrow B^{\prime}$. In addition, under the induced identification $\left.\left(\eta_{1}+\eta_{2}\right)\right|_{B^{\prime}}=\left.\eta_{1}\right|_{B^{\prime}}+\left.\eta_{2}\right|_{k[J]}$, addition of morphisms commutes with pushforward under $A^{\prime} \rightarrow B^{\prime}$.

In what follows, we will frequently drop the subscripts on $\alpha$ and + when there is little likelihood of confusion.

Proof. We first claim that an arbitrary choices of addition data for $A^{\prime}$ and $k[I]$ induces functors $+_{A^{\prime}, I}$ and $+_{k[I], I}$, with the isomorphism $\alpha$, and satisfying (i) as well as the first identity of (iv). The functors are given on objects by the choice of $\sigma_{*}\left(\eta_{1} * \eta_{2}\right)$, and by Proposition 2.31 we then also obtain an addition operation on morphisms. The compatibility of $*$ and pushforward with composition of morphisms implies that we obtain functors, as asserted, and the fact that both $*$ and pushforward are unique up to unique isomorphism implies that any two choices of $+_{A^{\prime}, I}$ differ by unique isomorphism. To check (i), for any $\eta \in \mathcal{S}_{k[I]}$, we construct a functor ${ }_{-}{ }_{A^{\prime}, I} \eta$ which is "inverse" to $\_+{ }_{A^{\prime}, I} \eta$, in the sense that the composition on either side is fully faithful, and satisfies $\left(\eta^{\prime}+\eta\right)-\eta \cong\left(\eta^{\prime}-\eta\right)+\eta \cong \eta^{\prime}$ for 
all $\eta^{\prime} \in \mathcal{S}_{A^{\prime}}$. We define $-{ }_{A^{\prime}, I} \eta$ using the map $\delta: A^{\prime} \times_{k} k[I] \rightarrow A^{\prime}$ given by $\delta(x, \pi(x)+i)=x-i$. For every $\eta^{\prime} \in \mathcal{S}_{A^{\prime}}$, choose a pushforward $\delta_{*}\left(\eta^{\prime} * \eta\right)$, and define the functor on morphisms by $\varphi \mapsto \delta_{*}\left(\varphi * \mathrm{id}_{\eta}\right)$. We then see that

$$
\begin{aligned}
\left({ }_{-}-\eta\right)+\eta & =\left(\delta_{*}\left({ }_{-} * \eta\right)\right)+\eta \\
& \preccurlyeq \sigma_{*}(\delta \times \mathrm{id})_{*}\left({ }_{-} * \eta * \eta\right) \\
& =\sigma_{*}(\delta \times \mathrm{id})_{*}(\mathrm{id} \times \Delta)_{*}\left({ }_{-} * \eta\right) \\
& =p_{1 *}\left(\_* \eta\right),
\end{aligned}
$$

and

$$
\begin{aligned}
\left({ }_{-}+\eta\right)-\eta & =\left(\sigma_{*}(\ldots * \eta)\right)-\eta \\
& \preccurlyeq \delta_{*}(\sigma \times \mathrm{id})_{*}(\ldots * \eta * \eta) \\
& =\delta_{*}(\sigma \times \mathrm{id})_{*}(\mathrm{id} \times \Delta)_{*}(\ldots * \eta) \\
& =p_{1 *}\left(\_* \eta\right),
\end{aligned}
$$

where $\Delta: k[I] \rightarrow k[I] \times_{k} k[I]$ is the diagonal map, and $F \preccurlyeq G$ denotes that the functor $F$ is a particular realization of $G$, where $G$ is defined only up to unique isomorphism. This proves the desired assertions.

The associativity isomorphism $\alpha$ is obtained similarly, except that we are forced to keep track of our unique isomorphisms. Given $\eta_{1}, \eta_{2}, \eta_{3}$, if we choose any $\eta_{1} * \eta_{2} * \eta_{3}$ with projection maps from $\eta_{1}, \eta_{2}, \eta_{3}$, we see that our prior choices of $\eta_{1} * \eta_{2}$ and $\eta_{2} * \eta_{3}$, with projection maps, induce unique maps $\eta_{1} * \eta_{2} \rightarrow \eta_{1} * \eta_{2} * \eta_{3}$ and $\eta_{2} * \eta_{3} \rightarrow \eta_{1} * \eta_{2} * \eta_{3}$ over $p_{12}$ and $p_{23}$ respectively: indeed, any $p_{12} *\left(\eta_{1} * \eta_{2} * \eta_{3}\right)$ gives a choice of $\eta_{1} * \eta_{2}$, which then differs by a unique isomorphism from our given choice, and composing the pushforward with the isomorphism gives us the desired map. The same works for $p_{23}$. A similar argument produces unique maps $\left(\eta_{1}+\eta_{2}\right) * \eta_{3} \rightarrow \eta_{1} * \eta_{2} * \eta_{3}$ and $\eta_{1} *\left(\eta_{2}+\eta_{3}\right) \rightarrow \eta_{1} * \eta_{2} * \eta_{3}$ lying over $\sigma \times$ id and id $\times \sigma$ respectively, and compatible with the previously constructed maps. We thus see that

$$
\begin{aligned}
\left(\eta_{1}+\eta_{2}\right)+\eta_{3} & =\sigma_{*}\left(\sigma_{*}\left(\eta_{1} * \eta_{2}\right) * \eta_{3}\right) \\
& \preccurlyeq \sigma_{*}\left((\sigma \times \mathrm{id})_{*}\left(\eta_{1} * \eta_{2} * \eta_{3}\right)\right) \\
& =\sigma_{*}^{(2)}\left(\eta_{1} * \eta_{2} * \eta_{3}\right),
\end{aligned}
$$

where $\sigma^{(2)}: A^{\prime} \times_{k} k[I] \times_{k} k[I] \rightarrow A^{\prime}$ is the map defined by

$$
\sigma^{(2)}\left(x, \pi(x)+i_{1}, \pi(x)+i_{2}\right)=x+i_{1}+i_{2} .
$$

But $\eta_{1}+\left(\eta_{2}+\eta_{3}\right)$ is then a different realization of $\sigma_{*}^{(2)}\left(\eta_{1} * \eta_{2} * \eta_{3}\right)$, so we obtain a unique isomorphism $\alpha_{\eta_{1}, \eta_{2}, \eta_{3}}$, which one checks is independent of the choice of $\eta_{1} * \eta_{2} * \eta_{3}$ and gives the desired isomorphism of functors. The first identity of (iv) is checked similarly, by expressing both $\left(\mathrm{id}_{\eta_{1}}+\alpha_{\eta_{2}, \eta_{3}, \eta_{4}}\right) \circ \alpha_{\eta_{1}, \eta_{2}+\eta_{3}, \eta_{4}} \circ$ 
$\left(\alpha_{\eta_{1}, \eta_{2}, \eta_{3}}+\mathrm{id}_{\eta_{4}}\right)$ and $\alpha_{\eta_{1}, \eta_{2}, \eta_{3}+\eta_{4}} \circ \alpha_{\eta_{1}+\eta_{2}, \eta_{3}, \eta_{4}}$ as the unique isomorphisms between two different pushforwards of the quadruple product $\eta_{1} * \eta_{2} * \eta_{3} * \eta_{4}$.

We next show that there exist choices of addition data for $A^{\prime}$ and $k[I]$ satisfying (ii), (iii), and the second identity of (iv) (which makes sense only if (iii) is assumed). However, we first observe that a choice satisfying condition (iii) necessarily also satisfies the second identity of (iv): as when checking the first identity of (iv), both $\alpha_{\eta_{1}, \eta_{2}, \eta_{3}} \circ \alpha_{\eta_{3}, \eta_{1}, \eta_{2}}$ and $\alpha_{\eta_{1}, \eta_{3}, \eta_{2}}$ can be realized as the unique isomorphisms between two choices of pushforwards of $\eta_{1} * \eta_{2} * \eta_{3}$, so must agree. Thus, it is enough to show we can choose our addition data so that (ii) and (iii) are satisfied. For (ii), we begin by observing that because $\zeta_{I}=s_{*}\left(\zeta_{0}\right)$ where $s: k \rightarrow k[I]$ is the structure map, we always have (up to unique isomorphism)

$$
\eta^{\prime}+\zeta_{I}=\sigma_{*}\left(\eta^{\prime} *\left(s_{*} \zeta_{0}\right)\right)=\sigma_{*}(\mathrm{id} \times(s \circ \pi))_{*}\left(\eta^{\prime}\right)=\mathrm{id}_{*} \eta^{\prime}=\eta^{\prime},
$$

so if we realize $\eta^{\prime} * \zeta_{I}$ as $(\mathrm{id} \times s)_{*} \eta^{\prime}$ in such a way that the composition map $\eta^{\prime} \rightarrow$ $\eta^{\prime} * \zeta_{I} \rightarrow \eta^{\prime}$ over $A^{\prime} \stackrel{\text { id } \times s}{\rightarrow} A^{\prime} \times_{k} k[I] \stackrel{p_{1}}{\rightarrow} A^{\prime}$ is the identity, we obtain a unique map $\eta^{\prime} \rightarrow \eta^{\prime} * \zeta_{I}$ over $\sigma$ such that the composition map $\eta^{\prime} \rightarrow \eta^{\prime} * \zeta_{I} \rightarrow \eta^{\prime}$ over $A^{\prime} \stackrel{\text { id } \times s}{\rightarrow} A^{\prime} \times_{k} k[I] \stackrel{\sigma}{\rightarrow} A^{\prime}$ is also the identity. One then checks that with this choice of $\eta^{\prime} * \zeta_{I}$ for each $\eta^{\prime}$, given $\varphi: \eta_{1}^{\prime} \rightarrow \eta_{2}^{\prime}$ we have $\varphi * \operatorname{id}_{\zeta_{I}}=(\mathrm{id} \times s)_{*} \varphi$, and therefore that we have that $-+\zeta_{I}$ is the identity functor, as desired.

We now verify that when $A^{\prime}=k[I]$, we may further make the necessary choices in such a way that commutativity holds. For this, we place a total ordering on the objects of $\mathcal{S}_{k[I]}$ such that $\zeta_{I}$ is minimal. Then, for every $\eta_{1}^{\prime} \geq \eta_{2}^{\prime}$, we fix choices of $\eta_{1}^{\prime} * \eta_{2}^{\prime}$ and $\sigma_{*}\left(\eta_{1}^{\prime} * \eta_{2}^{\prime}\right)$ arbitrarily, except that when $\eta_{2}^{\prime}=\zeta_{I}$ we make our choice as dictated above. For $\eta_{1}^{\prime}<\eta_{2}^{\prime}$, having already chosen $\eta_{2}^{\prime} * \eta_{1}^{\prime}$ and $\eta_{2}^{\prime}+\eta_{1}^{\prime}$, we set $\eta_{1}^{\prime} * \eta_{2}^{\prime}$ to be a choice of pushforward $\mathrm{sw}_{*} \eta_{2}^{\prime} * \eta_{1}^{\prime}$. We then choose $\eta_{1}^{\prime}+\eta_{2}^{\prime}=\eta_{2}^{\prime}+\eta_{1}^{\prime}$ as an object, with the map $\eta_{1}^{\prime} * \eta_{2}^{\prime} \rightarrow \eta_{1}^{\prime}+\eta_{2}^{\prime}$ over $\sigma$ chosen so that the map $\eta_{2}^{\prime} * \eta_{1}^{\prime} \rightarrow \eta_{1}^{\prime} * \eta_{2}^{\prime}$ over sw pushes forward under $\sigma$ to the identity map. One checks via a diagram chase that such a choice makes $+_{A^{\prime}, I}$ commutative not only on the level of objects but also of morphisms: given $f_{i}: \eta_{i}^{\prime} \rightarrow \mu_{i}^{\prime}$, if we denote by $\mathrm{sw}_{\eta^{\prime}}$ and $\mathrm{sw}_{\mu^{\prime}}$ respectively the pushforward (iso)morphisms $\eta_{1}^{\prime} * \eta_{2}^{\prime} \rightarrow \eta_{2}^{\prime} * \eta_{1}^{\prime}$ and $\mu_{1}^{\prime} * \mu_{2}^{\prime} \rightarrow \mu_{2}^{\prime} * \mu_{1}^{\prime}$ over sw, one checks that $\mathrm{sw}_{\mu^{\prime}}^{-1} \circ\left(f_{2} * f_{1}\right) \circ \mathrm{sw}_{\eta^{\prime}}$ satisfies the characterization of $f_{1} * f_{2}$, which yields the desired commutativity.

Finally, our functoriality assertion follows from basic properties of categories fibered in groupoids, and the uniqueness of $*$ in Proposition 2.31. Indeed, if we have $\eta_{1} * \eta_{2}$ with maps from $\eta_{1}$ and $\eta_{2}$, and choices of $\left.\eta_{1}\right|_{B^{\prime}} \rightarrow \eta_{1}$ and $\left.\eta_{2}\right|_{k[J]} \rightarrow \eta_{2}$ one checks directly from the definition of a category fibered in groupoids that for any choice of $\left.\left(\eta_{1} * \eta_{2}\right)\right|_{B^{\prime} \times_{k} k[J]} \rightarrow \eta_{1} * \eta_{2}$ there exist unique maps from $\left.\eta_{1}\right|_{B^{\prime}}$ and $\left.\eta_{2}\right|_{k[J]}$ to $\left.\left(\eta_{1} * \eta_{2}\right)\right|_{B^{\prime} \times_{k} k[J]}$ making everything commute. From Proposition 2.31 we then obtain a unique isomorphism $\left.\left.\left.\left(\eta_{1} * \eta_{2}\right)\right|_{B^{\prime} \times k} k[J] \rightarrow \eta_{1}\right|_{B^{\prime}} * \eta_{2}\right|_{k[J]}$, and composing with this isomorphism gives us a map $\left.\left.\eta_{1}\right|_{B^{\prime}} * \eta_{2}\right|_{k[J]} \rightarrow \eta_{1} * \eta_{2}$ over $A^{\prime} \times_{k} k[I] \rightarrow B^{\prime} \times_{k} k[J]$. Using the definition of a category fibered in groupoids once again, we obtain the desired unique map $\left.\eta_{1}\right|_{B^{\prime}}+\left.\eta_{2}\right|_{k[J]} \rightarrow \eta_{1}+\eta_{2}$ over $A^{\prime} \rightarrow B^{\prime}$, commuting with the prechosen pushforward maps $\eta_{1}+\eta_{2} \rightarrow \eta_{1} * \eta_{2}$ 
and $\left.\eta_{1}\right|_{B^{\prime}}+\left.\left.\left.\eta_{2}\right|_{k[J]} \rightarrow \eta_{1}\right|_{B^{\prime}} * \eta_{2}\right|_{k[J]}$. One then checks by chasing definitions that the desired functoriality for morphisms follows from the commutativity of all the involved maps.

This says, roughly speaking, that $\mathcal{S}_{k[I]}$ is always an "abelian group category", and that $\mathcal{S}_{k[I]}$ acts naturally on $\mathcal{S}_{A^{\prime}}$. We next show that in fact the action of $\mathcal{S}_{k[I]}$ factors through a collection of much "smaller" auxiliary categories $\mathcal{S}_{\eta, A^{\prime}}$ associated to $\mathcal{S}_{A^{\prime}}$, making each of the $\mathcal{S}_{\eta, A^{\prime}}$ into "pseudotorsor categories" for $\mathcal{S}_{k[I]}$.

Definition 2.34. Given $\eta \in \mathcal{S}_{A}$, let $\mathcal{S}_{\eta, A^{\prime}}$ be the category whose objects consist of pairs $\left(\eta^{\prime}, \varphi\right)$ with $\eta^{\prime} \in \mathcal{S}_{A^{\prime}}$, and $\varphi: \eta \rightarrow \eta^{\prime}$ lying over $A^{\prime} \rightarrow A$, and with morphisms $\left(\eta_{1}^{\prime}, \varphi_{1}\right) \rightarrow\left(\eta_{2}^{\prime}, \varphi_{2}\right)$ given by $\psi: \eta_{1}^{\prime} \rightarrow \eta_{2}^{\prime}$ in $\mathcal{S}_{A^{\prime}}$ with $\varphi_{2} \circ \psi=\varphi_{1}$.

Our basic result is then the following:

Proposition 2.35. For any $\eta \in \mathcal{S}_{A}$, the functor $+_{A^{\prime}, I}$ of Theorem 2.33 induces a natural functor (which we still denote by $+_{A^{\prime}, I}$ ) on $\mathcal{S}_{\eta, A^{\prime}}$, with any two choices of ${ }_{A^{\prime}, I}$ differing by an isomorphism of functors. The associativity isomorphism $\alpha$ induces a new isomorphism of functors which we still denote by $\alpha$, and $-{ }_{A^{\prime}, I} \zeta_{I}$ is still the identity functor.

Furthermore, if we choose any object $\left(\eta^{\prime}, \varphi\right) \in \mathcal{S}_{\eta, A^{\prime}}$, the induced functor

$$
\left(\eta^{\prime}, \varphi\right)+_{A^{\prime}, I}-: \mathcal{S}_{k[I]} \rightarrow \mathcal{S}_{\eta, A^{\prime}}
$$

is an equivalence of categories.

Proof. To define the functor induced by $+_{A^{\prime}, I}$ on $\mathcal{S}_{\eta, A^{\prime}}$, suppose we have $\left(\eta_{1}^{\prime}, \varphi_{1}\right) \in$ $\mathcal{S}_{\eta, A^{\prime}}$, and $\eta_{2} \in \mathcal{S}_{k[I]}$. We set $\left(\eta_{1}^{\prime}, \varphi_{1}\right)+\eta_{2}=\left(\eta_{1}^{\prime}+\eta_{2}, \varphi_{12}\right)$, where $\varphi_{12}$ is uniquely determined from the axioms of a category fibered in groupoids by the condition that the composed map $\eta \stackrel{\varphi_{1}}{\rightarrow} \eta_{1}^{\prime} \stackrel{q_{1}}{\rightarrow} \eta_{1}^{\prime} * \eta_{2}$ over $A^{\prime} \times_{k} k[I] \stackrel{p_{1}}{\rightarrow} A^{\prime} \rightarrow A$ agrees with $\eta \stackrel{\varphi_{12}}{\rightarrow} \eta_{1}^{\prime}+\eta_{2} \rightarrow \eta_{1}^{\prime} * \eta_{2}$ over $A^{\prime} \times_{k} k[I] \stackrel{\sigma}{\rightarrow} A^{\prime} \rightarrow A$. One checks easily that the addition of morphisms given by $+_{A^{\prime}, I}$ makes our new addition law on $\mathcal{S}_{\eta, A^{\prime}}$ into a functor. Moreover, we already know that two different choices of $+_{A^{\prime}, I}$ differ by a unique isomorphism of functors on $\mathcal{S}_{A^{\prime}}$, and it is routine to check that the relevant isomorphisms in $\mathcal{S}_{A^{\prime}}$ are in fact isomorphisms in $\mathcal{S}_{\eta, A^{\prime}}$, so it follows formally that we obtain isomorphisms of any two choices of the new addition functor as well. Similarly, we check directly from our construction that the isomorphisms in $\mathcal{S}_{A^{\prime}}$ given by $\alpha$ are isomorphisms in $\mathcal{S}_{\eta, A^{\prime}}$, and that $\zeta_{I}$ still acts as the identity.

To prove the equivalence assertion, we again construct the "inverse" functor $-\left(\eta^{\prime}, \varphi\right)+\ldots$ explicitly. Given $\left(\eta^{\prime}, \varphi\right) \in \mathcal{S}_{\eta, A^{\prime}}$, and any $\left(\eta_{1}^{\prime}, \varphi_{1}\right) \in \mathcal{S}_{\eta, A^{\prime}}$, we obtain $-\left(\eta^{\prime}, \varphi\right)+\left(\eta_{1}^{\prime}, \varphi_{1}\right) \in \mathcal{S}_{k[I]}$ as follows: we consider $\eta^{\prime} *_{\eta} \eta_{1}^{\prime}$, and write $\delta: A^{\prime} \times_{A} A^{\prime} \rightarrow k[I]$ for the map given by $(x, y) \mapsto \pi(x)+(y-x)$. We then set $-\left(\eta^{\prime}, \varphi\right)+\left(\eta_{1}^{\prime}, \varphi_{1}\right)=\delta_{*}\left(\eta^{\prime} *_{\eta} \eta_{1}^{\prime}\right)$. Here we fix any choices of $\eta^{\prime} *_{\eta} \eta_{1}^{\prime}$ and the resulting pushforwards. Given also $\left(\eta_{2}^{\prime}, \varphi_{2}\right)$, if we have a morphism $\psi: \eta_{1}^{\prime} \rightarrow \eta_{2}^{\prime}$ commuting with the $\varphi_{i}$, we obtain $\operatorname{id}_{\eta^{\prime}} *_{\eta} \psi$, and pushing forward under $\delta$ gives us a morphism $-\left(\eta^{\prime}, \varphi\right)+\left(\eta_{1}^{\prime}, \varphi_{1}\right) \rightarrow-\left(\eta^{\prime}, \varphi\right)+\left(\eta_{2}^{\prime}, \varphi_{2}\right)$. Compatibility of $*_{\eta}$ and 
pushforward with composition demonstrates that this defines a functor from $\mathcal{S}_{k[I]}$ to $\mathcal{S}_{\eta, A^{\prime}}$.

It remains to check that $-\left(\eta^{\prime}, \varphi\right)+\ldots$ is "inverse" to $\left(\eta^{\prime}, \varphi\right)+\ldots$, in the same sense as in the proof of Theorem 2.33 (i). But we see that

$$
\begin{aligned}
\left(\eta^{\prime}, \varphi\right)+\left(-\left(\eta^{\prime}, \varphi\right)+\ldots\right) & =\left(\eta^{\prime}, \varphi\right)+\left(\delta_{*}\left(\left(\eta^{\prime}, \varphi\right) *_{\eta}\right]_{)}\right) \\
& =\sigma_{*}\left(\left(\eta^{\prime}, \varphi\right) *\left(\delta_{*}\left(\left(\eta^{\prime}, \varphi\right) *_{\eta} \_\right)\right)\right) \\
& \left.\preccurlyeq \sigma_{*}(\mathrm{id} \times \delta)_{*}\left(\left(\eta^{\prime}, \varphi\right) *\left(\eta^{\prime}, \varphi\right) *_{\eta}\right)\right) \\
& =\sigma_{*}(\mathrm{id} \times \delta)_{*}\left((\Delta \times \mathrm{id})_{*}\left(\left(\eta^{\prime}, \varphi\right) *_{\eta} \_\right)\right) \\
& =p_{2 *}\left(\left(\eta^{\prime}, \varphi\right) *_{\eta}\right),
\end{aligned}
$$

and

$$
\begin{aligned}
-\left(\eta^{\prime}, \varphi\right)+\left(\left(\eta^{\prime}, \varphi\right)+\ldots\right) & =-\left(\eta^{\prime}, \varphi\right)+\left(\sigma_{*}\left(\left(\eta^{\prime}, \varphi\right) *-\right)\right) \\
& =\delta_{*}\left(\left(\eta^{\prime}, \varphi\right) *_{\eta}\left(\sigma_{*}\left(\left(\eta^{\prime}, \varphi\right) *-\right)\right)\right) \\
& \preccurlyeq \delta_{*}(\mathrm{id} \times \sigma)_{*}\left(\left(\eta^{\prime}, \varphi\right) *_{\eta}\left(\eta^{\prime}, \varphi\right) * \_\right) \\
& =\delta_{*}(\mathrm{id} \times \sigma)_{*}\left(\left(\Delta^{\prime} \times \mathrm{id}\right)_{*}\left(\left(\eta^{\prime}, \varphi\right) * \_\right)\right) \\
& =p_{2 *}\left(\left(\eta^{\prime}, \varphi\right) * \_\right),
\end{aligned}
$$

where $\Delta: A^{\prime} \rightarrow A^{\prime} \times_{k} A^{\prime}$ and $\Delta^{\prime}: A^{\prime} \rightarrow A^{\prime} \times_{A} A^{\prime}$ are the diagonal maps. Thus we conclude the desired statement.

As indicated by Corollary 2.30, this has many consequences, on the level of isomorphism classes of objects and on the level of (auto)morphisms. We next make a closer study of the behavior of our additive structure on morphisms. Here we mention that several of our statements, including that automorphism groups are abelian and canonically identified with one another, were stated by Grothendieck in a similar setting in Section 1.5 of [7]. He did not include any arguments, saying that the statements were "without doubt well known to category theorists."

Corollary 2.36. Given $\eta \in \mathcal{S}_{A}$, we have:

(i) given two morphisms $f: \eta_{1} \rightarrow \eta_{2}, g: \eta_{2} \rightarrow \eta_{3}$ in $\mathcal{S}_{k[I]}$, we have the identity $g+_{k[I], I} f=g \circ f+_{k[I], I} \mathrm{id}_{\eta_{2}}$;

(ii) in $\operatorname{Aut}\left(\zeta_{I}\right)$, composition agrees with $+_{k[I], I}$, and in particular $\operatorname{Aut}\left(\zeta_{I}\right)$ is an abelian group;

(iii) given $\eta^{\prime} \in \mathcal{S}_{\eta, A^{\prime}}$, the map $\operatorname{Aut}\left(\zeta_{I}\right) \rightarrow \operatorname{Aut}\left(\eta^{\prime}\right)$ induced by $\varphi \mapsto \mathrm{id}_{\eta^{\prime}}+{ }_{A^{\prime}, I} \varphi$ is an isomorphism of groups under composition. In particular, $\operatorname{Aut}\left(\eta^{\prime}\right)$ is abelian;

(iv) when $\eta^{\prime} \cong \eta^{\prime \prime}$ in $\mathcal{S}_{\eta, A^{\prime}}$, the identification of $\operatorname{Aut}\left(\eta^{\prime}\right)$ with $\operatorname{Aut}\left(\eta^{\prime \prime}\right)$ obtained via the above mutual isomorphisms with $\operatorname{Aut}\left(\zeta_{I}\right)$ agrees with the usual identification obtained by conjugating with a choice of isomorphism $\eta^{\prime} \stackrel{\sim}{\rightarrow} \eta^{\prime \prime}$; 
(v) given $\eta_{1} \in \mathcal{S}_{\eta, A^{\prime}}, \eta_{2}, \eta_{3} \in \mathcal{S}_{k[I]}$, and $\varphi_{i} \in \operatorname{Aut}\left(\eta_{i}\right)$ for $i=1,2,3$,

$$
\left(\varphi_{1}+\varphi_{2}\right)+\varphi_{3}=\varphi_{1}+\left(\varphi_{2}+\varphi_{3}\right)
$$

under the above identification of $\operatorname{Aut}\left(\left(\eta_{1}+\eta_{2}\right)+\eta_{3}\right)$ with $\operatorname{Aut}\left(\eta_{1}+\left(\eta_{2}+\eta_{3}\right)\right)$; (vi) given $\eta_{1} \in \mathcal{S}_{\eta, A^{\prime}}$ and $\eta_{2} \in \mathcal{S}_{k[I]}$, and $\varphi_{1}, \varphi_{2} \in \operatorname{Aut}\left(\zeta_{I}\right)$, we have

$$
\left(\mathrm{id}_{\eta_{1}+\eta_{2}}\right)+\left(\varphi_{1}+\varphi_{2}\right)=\left(\mathrm{id}_{\eta_{1}}+\varphi_{1}\right)+\left(\mathrm{id}_{\eta_{2}}+\varphi_{2}\right)
$$

as elements of $\operatorname{Aut}\left(\eta_{1}+\eta_{2}\right)$.

Note that in the above, automorphism groups for objects of $\mathcal{S}_{\eta, A^{\prime}}$ are the automorphism groups in $\mathcal{S}_{\eta, A^{\prime}}$, and not the automorphism groups of the underlying object of $\mathcal{S}_{A^{\prime}}$. That is, we consider automorphisms which restrict to the identity in $\operatorname{Aut}(\eta)$.

Proof. For (i), using commutativity and the fact that $+_{k[I], I}$ is a functor, we have

$$
\begin{aligned}
g \circ f+\mathrm{id}_{\eta_{2}}= & \left(g+\mathrm{id}_{\eta_{2}}\right) \circ\left(f+\mathrm{id}_{\eta_{2}}\right)=\left(g+\mathrm{id}_{\eta_{2}}\right) \circ\left(\mathrm{id}_{\eta_{2}}+f\right)=\left(g \circ \mathrm{id}_{\eta_{2}}\right) \\
& +\left(\operatorname{id}_{\eta_{2}} \circ f\right)=g+f .
\end{aligned}
$$

Statement (ii) then immediately follows from (i), since $+_{k[I], I} \mathrm{id}_{\zeta_{I}}$ acts as the identity on morphisms.

(iii) follows immediately from Proposition 2.35 , since if $\eta^{\prime}+{ }_{A^{\prime}, I}: \mathcal{S}_{\eta, A^{\prime}} \rightarrow$ $\mathcal{S}_{k[I]}$ is an equivalence of categories, it necessarily induces isomorphisms on automorphism groups.

To see (iv) we suppose we are given $f: \eta^{\prime} \stackrel{\sim}{\rightarrow} \eta^{\prime \prime}$, and $\varphi^{\prime} \in \operatorname{Aut}\left(\eta^{\prime}\right)$. Let $\varphi \in$ $\operatorname{Aut}\left(\zeta_{I}\right)$ be the unique automorphism with $\operatorname{id}_{\eta^{\prime}}+{ }_{A^{\prime}, I} \varphi=\varphi^{\prime}$, and set $\varphi^{\prime \prime} \in \operatorname{Aut}\left(\eta^{\prime \prime}\right)$ to be $\operatorname{id}_{\eta^{\prime \prime}}+{ }_{A^{\prime}, I} \varphi$. We wish to see that $\varphi^{\prime}=f^{-1} \circ \varphi^{\prime \prime} \circ f$. But using again that $+_{k[I], I}$ is a functor, we have

$$
\begin{aligned}
f^{-1} \circ \varphi^{\prime \prime} \circ f & =\left(f^{-1}+{ }_{A^{\prime}, I} \mathrm{id}_{\zeta_{I}}\right) \circ\left(\operatorname{id}_{\eta^{\prime \prime}}+{ }_{A^{\prime}, I} \varphi\right) \circ\left(f+_{A^{\prime}, I} \operatorname{id}_{\zeta_{I}}\right) \\
& =\left(f^{-1} \circ \operatorname{id}_{\eta^{\prime \prime}} \circ f\right)+{ }_{A^{\prime}, I} \varphi=\operatorname{id}_{\eta^{\prime}}+{ }_{A^{\prime}, I} \varphi=\varphi^{\prime},
\end{aligned}
$$

as desired.

(v) follows trivially from (iv), since we have

$$
\left(\varphi_{1}+\varphi_{2}\right)+\varphi_{3}=\alpha_{\eta_{1}, \eta_{2}, \eta_{3}}^{-1} \circ\left(\varphi_{1}+\left(\varphi_{2}+\varphi_{3}\right)\right) \circ \alpha_{\eta_{1}, \eta_{2}, \eta_{3}} .
$$

Finally, (vi) then follows by repeated application of (v), after expanding $\mathrm{id}_{\eta_{1}+\eta_{2}}=$ $\mathrm{id}_{\eta_{1}}+\mathrm{id}_{\eta_{2}}$.

In particular, we see that the "linearization" imposed by the deformation stack condition means that the infinitesimal automorphism group $A_{\mathcal{S}}$ is necessarily abelian.

We can now easily give the proof of Corollary 2.30 . 
Proof of Corollary 2.30. We first address $T_{I}$. The operation $+_{k[I], I}$ is commutative with identity $\zeta_{I}$ by construction. That it is associative on the level of isomorphism classes is clear from the existence of the isomorphisms $\alpha_{x, y, z}$. Moreover, the existence of inverses follows from the fact that addition of a given object gives an equivalence of categories. The structure is canonical because any two choices of the functor $+_{k[I], I}$ differ by isomorphisms, so induce the same operation on $T_{I}$. The desired statements for $A_{I}$ are simply Corollary 2.36 (ii). Finally, functoriality for both $T_{I}$ and $A_{I}$ follows from the functoriality statement of Theorem 2.33.

The assertions for $T_{\eta}, A^{\prime}$ then follow easily from Proposition 2.35. Indeed, $T_{\eta}, A^{\prime}$ is simply the set of isomorphism classes of $\mathcal{S}_{\eta, A^{\prime}}$, so we easily see that we have a map $T_{\eta, A^{\prime}} \times T_{I} \rightarrow T_{\eta, A^{\prime}}$, with $\zeta_{I}$ acting as the identity, and the existence of $\alpha$ implies that this map indeed defines a group action. Lastly, if $T_{\eta, A^{\prime}}$ is non-empty, we see that it is in fact a torsor from the assertion of Proposition 2.35 that addition induces an equivalence of categories.

We next address the corresponding statements for automorphisms. We first note that the general case follows immediately from the case that $\varphi=\mathrm{id}$, since for any $\varphi$ we have that $A_{\varphi, \eta^{\prime}}$ is naturally a pseudotorsor for $A_{\mathrm{id}, \eta^{\prime}}$. But the isomorphism $A_{I} \stackrel{\sim}{\rightarrow} A_{\mathrm{id}, \eta^{\prime}}$ is precisely Corollary 2.36 (iii).

Functoriality in both cases follows immediately from the functoriality assertion of Theorem 2.33.

\subsection{Linear structure}

Given a $k$-vector space $V$, having analyzed the additive structures associated to $\mathcal{S}_{k[V]}$ for a deformation stack $\mathcal{S}$, we may similarly define scalar multiplication maps over $k$, making $T_{V}$ and $A_{V}$ into $k$-vector spaces. Although it is possible to do this on a categorical level as we did with addition, expressing the proper conditions for associativity and distributivity isomorphisms becomes substantially more complicated, so we settle for the simpler task of defining vector space structures on $T_{V}$ and $A_{V}$.

Definition 2.37. Given $\lambda \in k$, let $m_{\lambda}: k[V] \rightarrow k[V]$ be defined by $a+v \mapsto a+\lambda v$, for $a \in k, v \in V$. We then define maps $m_{\lambda *}: T_{V} \rightarrow T_{V}$ and $m_{\lambda *}: A_{V} \rightarrow A_{V}$ by pushing forward under $m_{\lambda}$.

These maps define vector space structures:

Corollary 2.38. The sets $T_{V}$ and $A_{V}$ carry canonical vector space structures defined by $+_{k[V], V}$ and the $m_{\lambda}$ maps, with the addition law on $A_{V}$ agreeing with composition.

Proof. We have already seen in Corollary 2.30 that $+_{k[V], V}$ gives canonical abelian group structures on $T_{V}$ and $A_{V}$ and agrees with composition for $A_{V}$, so it remains to check that the scalar multiplication maps are canonically defined, and satisfy the appropriate vector space axioms. That they are canonically defined for $T_{V}$ is clear, since any two choices of $m_{\lambda *} \eta$ differ by unique isomorphism. For $A_{V}$, we 
define $m_{\lambda *}$ more precisely by choosing a pushforward map $\zeta_{V} \rightarrow \zeta_{V}$ over $m_{\lambda}$ (which we observe we can do). This pushforward map is well-defined only up to composition by an element of $A_{V}$, but the resulting pushforward of automorphisms is then conjugated by that element, which leaves the map on $A_{V}$ unchanged because $A_{V}$ is abelian.

We next check the vector space axioms on $T_{V}$. It is clear that for $\eta \in T_{V}$, we have $m_{1 *} \eta=\eta$, since $m_{1}$ is the identity map. Similarly, since $m_{\lambda \lambda^{\prime}}=m_{\lambda} \circ m_{\lambda^{\prime}}$, we have

$$
\begin{aligned}
m_{\lambda \lambda^{\prime} *} \eta & =\left(m_{\lambda} \circ m_{\lambda^{\prime}}\right)_{*} \eta \\
& =m_{\lambda *}\left(m_{\lambda^{\prime} *} \eta\right) .
\end{aligned}
$$

Next, given given $\lambda, \lambda^{\prime} \in k$ and $\eta \in T_{V}$, we have

$$
\begin{aligned}
\left(m_{\lambda *} \eta\right)+\left(m_{\lambda^{\prime} *} \eta\right) & =\sigma_{*}\left(\left(m_{\lambda *} \eta\right) *\left(m_{\lambda^{\prime} *} \eta\right)\right) \\
& =\sigma_{*}\left(m_{\lambda, \lambda^{\prime} *}(\eta * \eta)\right) \\
& =\sigma_{*}\left(m_{\lambda, \lambda^{\prime} *}\left(\Delta_{*} \eta\right)\right) \\
& =m_{\lambda+\lambda^{\prime} *}(\eta)
\end{aligned}
$$

where $\Delta: k[V] \rightarrow k[V] \times_{k} k[V]$ is the diagonal imbedding and $m_{\lambda, \lambda^{\prime}}$ is the map $k[V] \times_{k} k[V] \rightarrow k[V] \times_{k} k[V]$ given by $m_{\lambda}$ on the first factor and $m_{\lambda^{\prime}}$ on the second.

Similarly, given $\lambda \in k$ and $\eta, \eta^{\prime} \in T_{V}$, we have

$$
\begin{aligned}
m_{\lambda *}\left(\eta+\eta^{\prime}\right) & =m_{\lambda *}\left(\sigma_{*}\left(\eta * \eta^{\prime}\right)\right) \\
& =\sigma_{*}\left(m_{\lambda, \lambda *}\left(\eta * \eta^{\prime}\right)\right) \\
& =\sigma_{*}\left(\left(m_{\lambda *} \eta\right) *\left(m_{\lambda *} \eta^{\prime}\right)\right) \\
& =\left(m_{\lambda *} \eta\right)+\left(m_{\lambda *} \eta^{\prime}\right) .
\end{aligned}
$$

Finally, the vector space axioms on $A_{V}$ follow from precisely the same arguments.

The following lemma gives an important functoriality statement.

Lemma 2.39. A linear map $V \rightarrow W$ induces a linear map $T_{V} \rightarrow T_{W}$ under pushforward. Moreover, in this way we obtain a linear map $\operatorname{Hom}(V, W) \rightarrow$ Hom $\left(T_{V}, T_{W}\right)$.

Finally, the same is true if we replace $T_{V}$ and $T_{W}$ by $A_{V}$ and $A_{W}$.

Proof. Indeed, the linearity of the map $V \rightarrow W$ implies that the induced maps commute with the $\sigma$ and $m_{\lambda}$ used to define the vector space structure, and hence give linear maps $T_{V} \rightarrow T_{W}$ by simple diagram chases.

It is clear from the definitions that the induced map $\operatorname{Hom}(V, W) \rightarrow \operatorname{Hom}$ $\left(T_{V}, T_{W}\right)$ commutes with scalar multiplication, so we need only check that it also 
commutes with addition. That is, given $\eta \in T_{V}$, and $\varphi_{1}, \varphi_{2} \in \operatorname{Hom}(V, W)$, we need to check that

$$
\left(\varphi_{1}+\varphi_{2}\right)_{*}(\eta)=\varphi_{1 *}(\eta)+\varphi_{2 *}(\eta) .
$$

But this is easy enough: write $\eta^{\prime}$ for the object of $F_{\mathcal{S}}\left(k[W] \times_{k} k[W]\right)$ inducing $\varphi_{1 *}(\eta)$ and $\varphi_{2 *}(\eta)$ under the projection maps, so that $\varphi_{1 *}(\eta)+\varphi_{2 *}(\eta)=\sigma_{*}\left(\eta^{\prime}\right)$. Then one need only note that $\eta^{\prime}$ is the pushforward of $\eta$ under the map $k[V] \rightarrow$ $k[W] \times_{k} k[W]$ induced by $\varphi_{1}$ and $\varphi_{2}$, and that the composition of this map with $\sigma$ is precisely $\varphi_{1}+\varphi_{2}$.

Finally, the argument in the case of $A_{V}, A_{W}$ proceeds in exactly the same fashion.

We can now prove the following explicit descriptions of $T_{V}$ and $A_{V}$ :

Proposition 2.40. For any $k$-vector space $V$ we have canonical $k$-vector space isomorphisms

$$
T_{V} \cong T_{\mathcal{S}} \otimes_{k} V
$$

and

$$
A_{V} \cong A_{\mathcal{S}} \otimes_{k} V
$$

functorial in $V$.

Proof. We can define a natural map $T_{\mathcal{S}} \otimes_{k} V \rightarrow T_{V}$ induced by sending $\eta \otimes v$ to the image of $\eta$ under $\varphi_{v}: k[\epsilon] \rightarrow k[V]$ defined by $\epsilon \mapsto v$. One checks using Lemma 2.39 that this gives for fixed $v$ a linear map $T_{\mathcal{S}} \rightarrow T_{V}$, and for fixed $\eta$ a linear map $V \rightarrow T_{V}$, so we obtain a well-defined linear map from the tensor product. To check that we have an isomorphism, we need only check bijectivity. We choose a basis $v_{1}, \ldots, v_{d}$ of $V$, and factor our map through the isomorphism $T_{\mathcal{S}} \otimes V \stackrel{\sim}{\rightarrow} T_{\left(v_{1}\right)} \times \cdots \times T_{\left(v_{d}\right)}$, using the isomorphisms $T_{\mathcal{S}} \stackrel{\sim}{\rightarrow} T_{\left(v_{i}\right)}$ induced by $\varphi_{v_{i}}$. The map $T_{\left(v_{1}\right)} \times \cdots \times T_{\left(v_{d}\right)} \rightarrow T_{V}$ is then induced by the inclusions $\left(v_{i}\right) \rightarrow V$ together with the $d$-fold summation map $\sigma_{*}: \underbrace{T_{V} \times \cdots \times T_{V}}_{d \text { times }} \rightarrow T_{V}$; it thus suffices to check that this composition map is a bijection. But considering the diagram

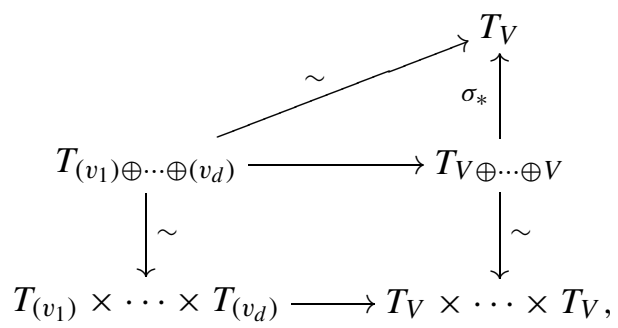

one checks from the definitions that everything commutes, and inductively uses the deformation stack axioms to show that the lower vertical arrows are isomorphisms. 
Our map $T_{\left(v_{1}\right)} \times \cdots \times T_{\left(v_{d}\right)} \rightarrow T_{V}$ is obtained from the diagram by inverting the appropriate isomorphism, so we see that it is a bijection, as desired.

Finally, to check functoriality, if we have $\phi: V \rightarrow W$, which by abuse of notation we also consider as a map $k[V] \rightarrow k[W]$, we know from Lemma 2.39 and the above that all maps in question are linear, so it suffices to check agreement on elements of the form $\eta \otimes v$. We therefore need to see that $\varphi_{\phi(v) *} \eta=\phi_{*}\left(\varphi_{v *} \eta\right)$. But we have $\phi \circ \varphi_{v}=\varphi_{\phi(v)}$, so this is trivial.

We next move on to the case of $A_{V}$; the argument is largely the same as above. Given a tensor $\varphi \otimes v$ with $\varphi \in A_{\mathcal{S}}$ and $v \in V$, we can use the induced map $i_{v}: k[\epsilon] \rightarrow k[V]$ sending $\epsilon$ to $v$ to obtain $i_{v *} \varphi \in \operatorname{Aut}\left(\zeta_{V}\right)$. This map can be extended additively to give a map $A_{\mathcal{S}} \otimes_{k} V \rightarrow \operatorname{Aut}\left(\zeta_{V}\right)$, and it follows from Lemma 2.39 that this map is well-defined and $k$-linear. Finally, one checks that this map is an isomorphism by choosing a basis $v_{1}, \ldots, v_{d}$ of $V$, and exploiting the description

$$
k[V] \cong k\left[v_{1}\right] \times_{k} k\left[v_{2}\right] \times_{k} \cdots \times_{k} k\left[v_{d}\right],
$$

as in the case of $T_{V}$. The argument for functoriality is likewise the same.

Applying the proposition and Corollary 2.30, we see that by writing any $A$ as a series of small extensions of $k$, we inductively conclude the following.

Corollary 2.41. If $T_{\mathcal{S}}=0$, then there is at most one isomorphism class in $\mathcal{S}_{A}$ over any $A$.

If $A_{\mathcal{S}}=0$, then $\operatorname{Aut}(\eta)=\{\mathrm{id}\}$ for every $\eta \in \mathcal{S}$. In particular, in this case $(\mathrm{H} 4)$ is satisfied for $F_{\mathcal{S}}$.

Note that the last assertion follows from Proposition 2.12.

One can often study $A_{\mathcal{S}}$ more directly than would be the case for more general automorphism groups in $\mathcal{S}$, and in particular one can sometimes show that $\mathcal{S}$ has no non-trivial automorphisms even in cases where automorphisms could a priori exist. See Corollary 3.25 below for an example.

Remark 2.42. Proposition 2.40, together with Corollary 2.30, also shows that if $\mathcal{S}$ is a deformation stack with an obstruction space, then $F_{\mathcal{S}}$ has a generalized tangentobstruction theory in the sense of Fantechi and Göttsche, [5, Definition 6.1.21].

\section{Geometric deformation stacks}

We now move on to the focus of our main theorems: deformation problems which are sufficiently geometric to be naturally associated to a scheme $X$. After defining geometric deformation stacks and studying their most basic properties, we use our results on deformation stacks to prove Theorems 1.1 and 1.2. We then discuss examples and special cases of these theorems. 


\subsection{Definitions and basic properties}

Many geometric deformation problems arise with more context than just a deformation functor or stack: specifically, such problems are naturally associated to some scheme, and given a morphism of schemes, we can pull back to obtain another deformation problem. We do not pursue this point of view in such generality: we are interested mainly in being able to restrict deformation problems to open subsets, so that we can formally talk about how to describe deformation problems globally in terms of their local behavior.

We let $X$ be a scheme over a field $k$, and we denote by $\operatorname{Zar}(X)$ the category of the Zariski topology on $X$.

Definition 3.1. Let $\mathcal{S}$ be a category fibered in groupoids over $\operatorname{Zar}(X) \times \operatorname{Art}$ $(\Lambda, k)^{\mathrm{opp}}$. We say that $\mathcal{S}$ is a geometric deformation stack if it satisfies:

(i) For each $A \in \operatorname{Art}(\Lambda, k), \mathcal{S}_{(,, A)}$ is a stack for the Zariski topology on $X$;

(ii) For each $\left.U \in \operatorname{Zar}(X), \mathcal{S}_{(U,-}\right)$ is a deformation stack.

Here $\mathcal{S}_{(-, A)}$ is the category fibered in groupoids over $\operatorname{Zar}(X)$ obtained as the subcategory of $\mathcal{S}$ consisting of objects lying over $(U, A)$ for some $U \in \operatorname{Zar}(X)$, and whose morphisms lie over $\left(\iota, \operatorname{id}_{A}\right)$ where $\iota$ is any morphism in $\operatorname{Zar}(X)$. We define $\mathcal{S}_{\left(U,{ }_{1}\right)}$ similarly. We assume we have chosen objects $\zeta_{0, U} \in \mathcal{S}_{U, k}$ for each $U$, and $\bar{\zeta}_{V, U} \in \mathcal{S}_{k[V], U}$ a pushforward of $\zeta_{0, U}$ for every finite-dimensional $k$-vector space $V$.

Remark 3.2. At first glance, working with the product category $\operatorname{Zar}(X) \times \operatorname{Art}$ $(\Lambda, k)^{\text {opp }}$ may appear unnatural, and one might be tempted to replace it with more familiar (but more complicated) categories, such as the infinitesimal site on $X$. However, it quickly becomes clear that the product category is indeed the best suited to treating the sort of questions we wish to address: the conditions we impose are very natural, and we are able to treat many deformation problems, such as deforming $X$ itself or deforming a sheaf on $X$, transparently in the same setting. Ultimately, the theory should be adaptable to working over various categories, and this could be useful for instance to place an $\mathcal{O}_{X}$-module structure on the sheaves of infinitesimal automorphisms and of local first-order deformations. However for the sort of analysis of tangent and obstruction spaces which is our primary goal, working with the product category seems to clearly offer the strongest results under the most general hypotheses.

We take a moment to consider the extra structure offered by a category fibered in groupoids $\mathcal{S}$ over a product category $C_{1} \times C_{2}$. Given an object $\eta$ over $\left(T_{1}, T_{2}\right)$, and a morphism $f_{1}: T_{1}^{\prime} \rightarrow T_{1}$ in $C_{1}$, we will often write $f_{1}^{*}(\eta)$ as an abbreviation for $\left(f_{1}, \operatorname{id}_{T_{2}}\right)^{*}(\eta)$, and similarly for morphisms in $C_{2}$. We can define pullbacks of morphisms somewhat more generally than we did initially:

Definition 3.3. Let $\eta, \eta^{\prime}$ be objects of $\mathcal{S}$ over $\left(T_{1}, T_{2}\right)$ and $\left(T_{1}^{\prime}, T_{2}\right)$ respectively, and let $\varphi: \eta^{\prime} \rightarrow \eta$ be a morphism lying over $\left(f_{1}, \operatorname{id}_{T_{2}}\right)$. Then given $f_{2}: T_{2}^{\prime} \rightarrow T_{2}$, we can define the pullback $f_{2}^{*}(\varphi): f_{2}^{*}\left(\eta^{\prime}\right) \rightarrow f_{2}^{*}(\eta)$ over $\left(f_{1}, \mathrm{id}_{T_{2}^{\prime}}\right)$ as the morphism 
obtained by applying (ii) of the definition of a category fibered in groupoids to the composed morphism $f_{2}^{*}\left(\eta^{\prime}\right) \rightarrow \eta^{\prime} \stackrel{\varphi}{\rightarrow} \eta$ and $f_{2}^{*}(\eta) \rightarrow \eta$. We make a similar definition for pullbacks under $f_{1}$ of morphisms lying over $\left(\mathrm{id}_{T_{1}}, f_{2}\right)$.

The following basic properties of pullback are checked from the definitions, with the first coming from the fact that $\left(f_{1}, \mathrm{id}\right) \circ\left(\mathrm{id} f_{2}\right)=\left(f_{1}, f_{2}\right)=\left(\mathrm{id}, f_{2}\right) \circ$ $\left(f_{1}, \mathrm{id}\right)$, and the rest checked directly as in Proposition 2.6.

Proposition 3.4. We have:

(i) Pullbacks in different variables commute: given $\eta$ in $\mathcal{S}_{\left(T_{1}, T_{2}\right)}$ and $f_{i}: T_{i}^{\prime} \rightarrow$ $T_{i}$ for $i=1,2$, we have $f_{1}^{*}\left(f_{2}^{*}(\eta)\right)=f_{2}^{*}\left(f_{1}^{*}(\eta)\right)$.

(ii) Pullback of morphisms commutes with composition: given $T_{2}^{\prime \prime} \stackrel{f_{2}^{\prime}}{\rightarrow} T_{2}^{\prime} \stackrel{f_{2}}{\rightarrow} T_{2}$ in $C_{2}$, and $f_{1}: T_{1}^{\prime} \rightarrow T_{1}$ in $C_{1}$, and $\eta, \eta^{\prime}$ in $\mathcal{S}$ over $\left(T_{1}, T_{2}\right),\left(T_{1}^{\prime}, T_{2}\right)$ respectively, with a morphism $\varphi: \eta^{\prime} \rightarrow \eta$ over $\left(f_{1}\right.$, id), we have $f_{2}^{\prime *}\left(f_{2}^{*}(\varphi)\right)=$ $\left(f_{2}^{\prime} \circ f_{2}\right)^{*}(\varphi)$.

(iii) Pullback of morphisms commutes with composition: given $f_{2}: T_{2}^{\prime} \rightarrow T_{2}$ in $C_{2}$, and $T_{1}^{\prime \prime} \stackrel{f_{1}^{\prime}}{\rightarrow} T_{1}^{\prime} \stackrel{f_{1}}{\rightarrow} T_{1}$ in $C_{1}$, and $\eta, \eta^{\prime}, \eta^{\prime \prime}$ in $\mathcal{S}$ over $\left(T_{1}, T_{2}\right),\left(T_{1}^{\prime}, T_{2}\right)$, $\left(T_{1}^{\prime \prime}, T_{2}\right)$ respectively, with morphisms $\varphi: \eta^{\prime} \rightarrow \eta$ and $\varphi^{\prime}: \eta^{\prime \prime} \rightarrow \eta^{\prime}$ over $\left(f_{1}, \mathrm{id}\right)$ and $\left(f_{1}^{\prime}\right.$, id) respectively, we have $f_{2}^{*}\left(\varphi \circ \varphi^{\prime}\right)=f_{2}^{*}(\varphi) \circ f_{2}^{*}\left(\varphi^{\prime}\right)$.

We use the additive structure of Section 2.4 on each open subset $U$ of $X$ to define an additive structure on $\mathcal{S}$, which in the case that $A^{\prime}=k[I]$ gives a Picard stack in the sense of Deligne (see [3, Definition 1.4.5 of Expose XVIII]).

Proposition 3.5. Any choices of addition data for $A^{\prime}$ and (if $\left.A^{\prime} \neq k[I]\right)$ for $k[I]$ over every $U \subseteq X$, each satisfying the conditions of Theorem 2.33, induces an additive structure consisting of functors

$$
+_{A^{\prime}, I}: \mathcal{S}_{\left(\ldots, A^{\prime}\right)} \times \operatorname{Zar}(X) \mathcal{S}_{\left(\_, k[I]\right)} \rightarrow \mathcal{S}_{\left(\ldots, A^{\prime}\right)}
$$

and

$$
+_{k[I], I}: \mathcal{S}_{(\ldots, k[I])} \times \operatorname{Zar}(X) \mathcal{S}_{(\ldots, k[I])} \rightarrow \mathcal{S}_{(\ldots, k[I])}
$$

and an isomorphism of functors

$$
\alpha:\left({ }_{A^{\prime}, I} \_\right)+_{A^{\prime}, I} \underset{\sim}{\rightarrow}+_{A^{\prime}, I}\left({ }_{k[I], I} \_\right)
$$

such that:

(i) for any $U \in \operatorname{Zar}(X)$ and $\eta \in \mathcal{S}_{U, k[I]}$, the functor $\_+{ }_{A^{\prime}, I} \eta: \mathcal{S}_{U, A^{\prime}} \rightarrow \mathcal{S}_{U, A^{\prime}}$ is an equivalence of categories;

(ii) $+_{k[I], I}$ is commutative, in the sense that $+_{k[I], I}=+_{k[I], I} \circ \mathrm{sw}$, where sw : $\mathcal{S}_{(\ldots, k[I])} \times \operatorname{Zar}(X) \mathcal{S}_{(\ldots, k[I])} \rightarrow \mathcal{S}_{(\ldots, k[I])} \times \operatorname{Zar}(X) \mathcal{S}_{(,, k[I])}$ is the functor switching factors; 
(iii) for every $U \in \operatorname{Zar}(X)$, we have that $\_{ }_{A^{\prime}, I} \zeta_{I, U}: \mathcal{S}_{U, A^{\prime}} \rightarrow \mathcal{S}_{U, A^{\prime}}$ is the identity functor;

(iv) for any $U \in \operatorname{Zar}(X)$ and $\eta_{1}, \eta_{2}, \eta_{3}, \eta_{4} \in \mathcal{S}_{U, k[I]}$, we have the identities

$\left(\mathrm{id}_{\eta_{1}}+\alpha_{\eta_{2}, \eta_{3}, \eta_{4}}\right) \circ \alpha_{\eta_{1}, \eta_{2}+\eta_{3}, \eta_{4}} \circ\left(\alpha_{\eta_{1}, \eta_{2}, \eta_{3}}+\mathrm{id}_{\eta_{4}}\right)=\alpha_{\eta_{1}, \eta_{2}, \eta_{3}+\eta_{4}} \circ \alpha_{\eta_{1}+\eta_{2}, \eta_{3}, \eta_{4}}$

and

$$
\alpha_{\eta_{1}, \eta_{2}, \eta_{3}} \circ \alpha_{\eta_{3}, \eta_{1}, \eta_{2}}=\alpha_{\eta_{1}, \eta_{3}, \eta_{2}} .
$$

In the above and hereafter, we denote by $\alpha_{\eta_{1}, \eta_{2}, \eta_{3}}:\left(\eta_{1}+\eta_{2}\right)+\eta_{3} \stackrel{\sim}{\rightarrow} \eta_{1}+\left(\eta_{2}+\eta_{3}\right)$ the isomorphism obtained from $\alpha$.

Such an addition law is canonical up to unique isomorphism of functors.

Here, the category $\mathcal{S}_{\left(-, A^{\prime}\right)} \times \operatorname{Zar}(X) \mathcal{S}_{(-, k[I])}$ has objects consisting of pairs of objects in $\left.\mathcal{S}_{(}, A^{\prime}\right)$ over a given $U \in \operatorname{Zar}(\bar{X})$, and morphisms pairs of morphisms lying over a given inclusion in $\operatorname{Zar}(X)$.

Proof. We first need to see that the addition data define the desired functor $+_{A^{\prime}, I}$ : $\mathcal{S}_{\left(\_, A^{\prime}\right)} \times \mathcal{S}_{(\ldots, k[I])} \rightarrow \mathcal{S}_{\left(\_, A^{\prime}\right)}$. We clearly obtain a functor on the level of objects, and also for morphisms over a fixed $U \in \operatorname{Zar}(X)$, simply by restricting to $U$.

What remains to define is addition of arbitrary pairs of morphisms: given $U^{\prime} \subseteq$ $U, \eta_{1} \in \mathcal{S}_{U^{\prime}, A^{\prime}}, \eta_{2} \in \mathcal{S}_{U^{\prime}, k[I]}, \mu_{1} \in \mathcal{S}_{U, A^{\prime}}$ and $\mu_{2} \in \mathcal{S}_{U, k[I]}$, together with $\varphi_{i}$ : $\eta_{i} \rightarrow \mu_{i}$ over $U^{\prime} \rightarrow U$, we need to define $\varphi_{1}+{ }_{A^{\prime}, I} \varphi_{2}$ so that ${ }_{A^{\prime}, I}$ is a functor. We have already chosen $\eta_{1} * \eta_{2}$ and $\mu_{1} * \mu_{2}$, and their pushforwards $\eta_{1}+\eta_{2}$ and $\mu_{1}+\mu_{2}$, and we claim there exists a unique $\varphi_{1} * \varphi_{2}: \eta_{1} * \eta_{2} \rightarrow \mu_{1} * \mu_{2}$ such that the restrictions to $A^{\prime}$ and $k[I]$ recover $\varphi_{1}$ and $\varphi_{2}$ respectively. We first show that there exists some map $\varphi: \eta_{1} * \eta_{2} \rightarrow \mu_{1} * \mu_{2}$ lying over $U^{\prime} \rightarrow U$. Indeed, if we make any choice of pullback $\left.\left(\mu_{1} * \mu_{2}\right)\right|_{U^{\prime}} \rightarrow \mu_{1} * \mu_{2}$, we can use the $\varphi_{i}$ as identifications $\eta_{i}=\left.\mu_{i}\right|_{U^{\prime}}$, and we therefore obtain maps $\left.\left(\mu_{1} * \mu_{2}\right)\right|_{U^{\prime}} \rightarrow \eta_{i}$ over $A^{\prime} \times_{k} k[I] \rightarrow A^{\prime}$ and $A^{\prime} \times_{k} k[I] \rightarrow k[I]$. This realizes $\left.\left(\mu_{1} * \mu_{2}\right)\right|_{U^{\prime}}$ as $\eta_{1} * \eta_{2}$, so we obtain a (unique) isomorphism with our chosen $\eta_{1} * \eta_{2}$, and composing the isomorphism with the pullback map gives the desired map $\varphi$.

We now show that we have a unique $\varphi_{1} * \varphi_{2}$. The definition of a category fibered in groupoids implies that morphisms $\eta_{1} * \eta_{2} \rightarrow \mu_{1} * \mu_{2}$ over $U^{\prime} \rightarrow U$ are in bijection with $\operatorname{Aut}_{U^{\prime}}\left(\eta_{1} * \eta_{2}\right)$, and similarly for maps $\eta_{i} \rightarrow \mu_{i}$ and $\operatorname{Aut}_{U^{\prime}}\left(\eta_{i}\right)$. Thus, if $\left.\varphi\right|_{A^{\prime}}$ differs from $\varphi_{1}$ by $\psi_{1} \in \operatorname{Aut}_{U^{\prime}}\left(\eta_{1}\right)$, and $\left.\varphi\right|_{k[I]}$ differs from $\varphi_{2}$ by $\psi_{2} \in$ $\operatorname{Aut}_{U^{\prime}}\left(\eta_{2}\right)$, by the deformation stack axioms there is a unique $\psi \in \operatorname{Aut}_{U^{\prime}}\left(\eta_{1} * \eta_{2}\right)$ restricting to $\psi_{1}$ and $\psi_{2}$, and setting $\varphi_{1} * \varphi_{2}=\varphi \circ \psi$ yields the desired unique map. We then set $\varphi_{1}+\varphi_{2}$ to be the restriction under $\sigma$ of $\varphi_{1} * \varphi_{2}$, given the choices of $\eta_{1}+\eta_{2}$ and $\mu_{1}+\mu_{2}$. The uniqueness of this construction immediately implies that it commutes with composition, so we obtain the desired functor $+_{A^{\prime}, I}$.

We then obtain the isomorphism $\alpha$ from Theorem 2.33, since any given $\alpha_{\eta_{1}, \eta_{2}, \eta_{3}}$ occurs over a single $U$. For the same reason, properties (i), (iii), and (iv) follow directly from Theorem 2.33. Finally, (ii) is automatically satisfied on the level of objects, and one checks that it also works for morphisms exactly as before. Since our choices are made exactly as in Theorem 2.33 , the assertion that two such choices differ by a unique isomorphism also follows from Theorem 2.33 . 
We obtain the following corollary on additivity of pullbacks:

Corollary 3.6. Given $U^{\prime} \subseteq U$ in $\operatorname{Zar}(X)$, and objects $\eta_{1} \in \mathcal{S}_{U, A^{\prime}}, \eta_{2} \in \mathcal{S}_{U, k[I]}$, and choices of pullbacks $\left.\eta_{1}\right|_{U^{\prime}} \rightarrow \eta_{1},\left.\eta_{2}\right|_{U^{\prime}} \rightarrow \eta_{2}$, the $+_{A^{\prime}, I}$ functor gives a canonical pullback map $\left.\eta_{1}\right|_{U^{\prime}}+\left.\eta_{2}\right|_{U^{\prime}} \rightarrow \eta_{1}+\eta_{2}$.

Given also $\mu_{1} \in \mathcal{S}_{U, A^{\prime}}, \mu_{2} \in \mathcal{S}_{U, k[I]}$, and choices of pullbacks $\left.\mu_{1}\right|_{U^{\prime}} \rightarrow \mu_{1}$, $\left.\mu_{2}\right|_{U^{\prime}} \rightarrow \mu_{2}$, if we use the induced pullback map $\left.\mu_{1}\right|_{U^{\prime}}+\left.\mu_{2}\right|_{U^{\prime}} \rightarrow \mu_{1}+\mu_{2}$, then for any $f_{1}: \eta_{1} \rightarrow \mu_{1}$ in $\mathcal{S}_{U, A^{\prime}}$ and $f_{2}: \eta_{2} \rightarrow \mu_{2}$ in $\mathcal{S}_{U, k[I]}$, we have the indentity

$$
\left.\left(f_{1}+f_{2}\right)\right|_{U^{\prime}}=\left.f_{1}\right|_{U^{\prime}}+\left.f_{2}\right|_{U^{\prime}}
$$

Proof. It is clear from the definitions that $+_{A^{\prime}, I}$ gives a canonical pullback map $\left.\eta_{1}\right|_{U^{\prime}}+\left.\eta_{2}\right|_{U^{\prime}} \rightarrow \eta_{1}+\eta_{2}$, simply as the sum of $\left.\eta_{1}\right|_{U^{\prime}} \rightarrow \eta_{1}$ and $\left.\eta_{2}\right|_{U^{\prime}} \rightarrow \eta_{2}$. For addition of morphisms, the commutative diagrams defining $\left.f_{1}\right|_{U^{\prime}}$ and $\left.f_{2}\right|_{U^{\prime}}$ yield a commutative diagram in $\mathcal{S}_{\left(\ldots, A^{\prime}\right)} \times \operatorname{Zar}(X) \mathcal{S}_{(-, k[I])}$, and the fact that $+_{A^{\prime}, I}$ is a functor then shows that $\left.f_{1}\right|_{U^{\prime}}+\left.f_{2}\right|_{U^{\prime}}$ satisfies the commutativity condition defining $\left.\left(f_{1}+f_{2}\right)\right|_{U^{\prime}}$.

Our observations that the functor associated to a deformation stack is always a deformation functor, and that ( $\mathrm{H} 4)$ can be understood concretely in terms of automorphisms, reduces representability questions down to (H3), which is to say, to understanding tangent spaces. Thus, we will be mainly interested in describing tangent and obstruction spaces to a geometric deformation stack.

Definition 3.7. Given a geometric deformation stack $\mathcal{S}$, we define the tangent space $T_{\mathcal{S}}$ of $\mathcal{S}$, a successive obstruction theory for $\mathcal{S}$ taking values in $V_{1}, \ldots, V_{n}$, and an obstruction space for $\mathcal{S}$ to be the tangent space of $\mathcal{S}_{(X,-)}$, a successive obstruction theory for $\left.\mathcal{S}_{(X,}\right)$, and an obstruction space for $\mathcal{S}_{\left(X,{ }_{-}\right)}$respectively.

Lemma 3.8. Let $\mathcal{S}$ be a geometric deformation stack, $U_{1} \subseteq U_{2} \in \operatorname{Zar}(X)$, and $V$ a k-vector space. The maps $T_{\mathcal{S}_{\left(U_{2},{ }_{-}\right)}, V} \rightarrow T_{\mathcal{S}_{\left(U_{1},{ }_{-}\right)}, V}$ and $A_{\mathcal{S}_{\left(U_{2},{ }_{-}\right)}, V} \rightarrow A_{\mathcal{S}_{\left(U_{1},{ }^{\prime}\right.}, V}$ induced by pullback from $U_{2}$ to $U_{1}$ are linear maps of $k$-vector spaces.

Proof. That the maps are additive follows from Corollary 3.6. They also commute with scalar multiplication because it is defined in terms of pushforwards along ring homomorphisms, and since we are simply working over the product category $\operatorname{Zar}(X) \times \operatorname{Art}(\Lambda, k)^{\mathrm{opp}}$, such pushforwards commute with the restriction from $U_{2}$ to $U_{1}$.

As mentioned earlier, our main results on tangent and obstruction spaces will take the form of describing the spaces in terms of local information. The main tools which will allow this are the following sheaves of $k$-vector spaces on $X$ :

Definition 3.9. Given a geometric deformation stack $\mathcal{S}$, denote by $\mathcal{T}_{\mathcal{S}}$ and $\mathcal{A}_{\mathcal{S}}$ respectively the tangent sheaf of $\mathcal{S}$, given as the sheafification of the presheaf $U \mapsto T_{\left.\mathcal{S}_{(U,-}\right)}$, and the infinitesimal automorphism sheaf $U \mapsto A_{\mathcal{S}_{(U,-)}}$. By Lemma 3.8, these are both sheaves of $k$-vector spaces. 
Similarly, for each $k$-vector space $V$, let $\mathcal{T}_{\mathcal{S}, V}$ and $\mathcal{A}_{\mathcal{S}, V}$ be the sheaves of $k$ vector spaces given by the sheafication of $U \mapsto T_{\left.\mathcal{S}_{(U,-}\right), V}$, and by $U \mapsto A_{\mathcal{S}_{(U,-)}, V}$ respectively.

In order to study lifts of objects over small extensions, it will also be convenient to introduce the following:

Notation 3.10. Given a small extension $A^{\prime} \rightarrow A$ with kernel $I$, and $\eta \in \mathcal{S}_{(X, A)}$, fix choices of restrictions $\left.\eta\right|_{U}$ for all $U \in \operatorname{Zar}(X)$, and denote by $\mathcal{S}_{\eta, A^{\prime}}$ the stack over $\operatorname{Zar}(X)$ whose objects consist of triples $\left(U, \eta^{\prime}, \varphi\right)$, with $U \in \operatorname{Zar}(X), \eta^{\prime} \in \mathcal{S}_{U, A^{\prime}}$, and $\varphi:\left.\eta\right|_{U} \rightarrow \eta^{\prime}$ lying over $A^{\prime} \rightarrow A$. Morphisms $\psi:\left(U_{1}, \eta_{1}^{\prime}, \varphi_{1}\right) \rightarrow\left(U_{2}, \eta_{2}^{\prime}, \varphi_{2}\right)$ consist of a morphism $\eta_{1}^{\prime} \rightarrow \eta_{2}^{\prime}$ in $\left.\mathcal{S}_{(}, A^{\prime}\right)$ which lies over an inclusion $U_{1} \rightarrow U_{2}$ and which satisfies $\psi \circ \varphi_{1}=\varphi_{2} \circ r$, where $r:\left.\left.\eta\right|_{U_{1}} \rightarrow \eta\right|_{U_{2}}$ is the canonical map.

We also let $T_{\eta, A^{\prime}, U}$ be the set of isomorphism classes of $\left(\mathcal{S}_{\eta, A^{\prime}}\right)_{U}$, and $\mathcal{T}_{\eta, A^{\prime}}$ be the sheaf of $\mathcal{T}_{I}$-pseudotorsors obtained as the sheafification of the presheaf given by $U \mapsto T_{\eta, A^{\prime}, U}$.

Finally, for the statements of our main theorems in full generality, it will be helpful to have the notion of a local obstruction sheaf also:

Definition 3.11. We say that a sheaf of $k$-vector spaces $\mathcal{O} b$ on $X$ is a local obstruction sheaf if we have also the data, for each $U \in \operatorname{Zar}(X)$, each small extension $A^{\prime} \rightarrow A$ in $\operatorname{Art}(\Lambda, k)$ with kernel $I$, and each $\eta \in \mathcal{S}_{(U, A)}$, of an element $\mathrm{ob}_{\eta, A^{\prime}} \in \mathcal{O} b(U) \otimes_{k} I$, compatible with restriction in $\operatorname{Zar}(X)$ and satisfying the functoriality condition of an obstruction space given in Definition 2.13, and such that $\mathrm{ob}_{\eta, A^{\prime}}=0$ if and only if there exists an open cover of $U$ by $\left\{U_{i}\right\}$, and $\eta_{i}^{\prime} \in \mathcal{S}_{\left(U_{i}, A^{\prime}\right)}$ such that $\left.\left.\eta_{i}^{\prime}\right|_{A} \cong \eta\right|_{U_{i}}$ for all $i$.

\subsection{Proof of the main theorems}

We now move on to the proofs of our main theorems. Given our results on deformation stacks, the proofs follow the usual proofs used in standard examples of deformation problems, and are fundamentally quite intuitive: we pass from local to global using open covers and gluing along intersections. The main construction for both the tangent space exact sequence and the final obstruction space in Theorem 1.1 is the following.

Proposition 3.12. Fix a small extension $A^{\prime} \rightarrow A$, with kernel $I$, and $\eta \in \mathcal{S}_{(X, A)}$. Then the map of sets induced by sheafification of $U \mapsto T_{\eta, A^{\prime}, U}$ can be extended to the following very short exact sequence:

$$
T_{\eta, A^{\prime}, X} \rightarrow \Gamma\left(X, \mathcal{T}_{\eta, A^{\prime}}\right) \rightarrow H^{2}\left(X, \mathcal{A}_{I}\right),
$$

meaning that we have maps of sets, with the kernel of the second map equal to the image of the first. This sequence is functorial in that for any small extension $B^{\prime} \rightarrow B$ with kernel $J$, and map $\phi: A^{\prime} \rightarrow B^{\prime}$ mapping $I$ into $J$, the above maps commute with the maps induced by $\phi$, restriction of $\eta$ to $B$, and $I \rightarrow J$. 
Furthermore, the constructed map $\Gamma\left(X, \mathcal{T}_{\eta}, A^{\prime}\right) \rightarrow H^{2}\left(X, \mathcal{A}_{I}\right)$ is compatible with addition, and in the case that $A^{\prime}=k[I]$ and $A=k$, our construction yields $a$ $k$-linear map $\Gamma\left(X, \mathcal{T}_{I}\right) \rightarrow H^{2}\left(X, \mathcal{A}_{I}\right)$.

Before giving a more elementary proof along the lines of standard Čech cohomology arguments, we mention a stack-theoretic proof of the proposition: given a section $s \in H^{0}\left(X, \mathcal{T}_{\eta, A^{\prime}}\right)$, one can define a substack $\mathcal{S}_{s}$ of $\mathcal{S}_{\eta, A^{\prime}}$ consisting of the full subcategory containing every object locally isomorphic to the objects defining $s$. This is then an $\mathcal{A}_{I}$-gerbe over $X$, whose class is an element of $H^{2}\left(X, \mathcal{A}_{I}\right)$, giving us the desired map. Exactness is a consequence of the fact that the class of a gerbe is trivial if and only if the gerbe has a global section, which is to say if and only if $s$ actually came from an element of $T_{\eta}, A^{\prime}$.

Our more elementary proof follows the ideas of classical Čech cohomology, but cannot rely solely on them, and the reason is quite simple: given a presheaf $\mathcal{F}$ on a topological space $X$ with sheafification $\tilde{\mathcal{F}}$, and a global section $\rho \in \tilde{\mathcal{F}}(X)$, there is not any reason to think that we can find an open cover $\left\{U_{i}\right\}$ of $X$ on which $\rho$ is the image of some $\left\{\rho_{i} \in \mathcal{F}\left(U_{i}\right)\right\}$ which agree on the $U_{i} \cap U_{j}$. Certainly, $\rho$ is the image of some $\rho_{i}$, but we see that a priori we have equality of $\rho_{i}$ and $\rho_{j}$ only locally on $U_{i} \cap U_{j}$, and it is not necessarily possible to refine the $U_{i}$ to obtain the desired equality on all of $U_{i} \cap U_{j}$. See Example B.6 below for one such presheaf. We therefore work with the simplest generalization of Čech cohomology in the direction of hypercovers, which is enough to describe the sheaf cohomology group $H^{2}$ on an arbitrary topological space with an arbitrary sheaf of groups. We give a self-contained account of this approach to cohomology in Appendix B below.

Proof. We construct the map $\Gamma\left(X, \mathcal{T}_{\eta}, A^{\prime}\right) \rightarrow H^{2}\left(X, \mathcal{A}_{I}\right)$ as follows: given $\rho \in$ $\Gamma\left(X, \mathcal{T}_{\eta} A^{\prime}\right)$, let $\left\{U_{i}\right\}$ be an open cover small enough so that $\rho$ is represented by sections $\left(\rho_{i}, \varphi_{i}\right) \in T_{\eta, A^{\prime}, U_{i}}$, with $\rho_{i} \in \mathcal{S}_{\left(U_{i}, A^{\prime}\right)}$ and $\varphi_{i}:\left.\eta\right|_{U_{i}} \rightarrow \rho_{i}$ lying over $A^{\prime} \rightarrow A$. For each $i_{0} \neq i_{1}$, let $\left\{U_{i_{0}, i_{1}, j}\right\}_{j}$ be an open cover of $U_{i_{0}, i_{1}}$ such that on each $U_{i_{0}, i_{1}, j}$ there exists an isomorphism $\varphi_{i_{0}, i_{1}, j}:\left.\left.\rho_{i_{0}}\right|_{U_{i_{0}, i_{1}, j}} \rightarrow \rho_{i_{1}}\right|_{U_{i_{0}, i_{1}, j}}$ commuting with $\left.\varphi_{i_{0}}\right|_{U_{i_{0}, i_{1}, j}}$ and $\left.\varphi_{i_{1}}\right|_{U_{i_{0}, i_{1}, j}}$. We choose such $\varphi_{i_{0}, i_{1}, j}$, imposing that $\varphi_{i_{0}, i_{1}, j}=$ $\varphi_{i_{1}, i_{0}, j}^{-1}$, and we also set the convention that for $i_{0}=i_{1}$, we take the one-set cover $U_{i_{0}}$, with $\varphi_{i_{0}, i_{0},-}=\mathrm{id}$. We then obtain a 2-cochain $\rho^{\prime}$ with coefficients in $\mathcal{A}_{I}$, in the sense of Appendix B, uniquely characterized via Corollary 2.30 by the identity, for $\mathbf{i}=\left(i_{0}, i_{1}, i_{2}\right)$ and $\mathbf{j}=\left(j_{1,2}, j_{0,2}, j_{0,1}\right)$,

$$
\operatorname{id}_{\rho_{i}}+\rho_{\mathbf{i}, \mathbf{j}}^{\prime}=\left.\left.\left.\varphi_{i_{2}, i_{0}, j_{0,2}}\right|_{U_{\mathbf{i}, \mathbf{j}}} \circ \varphi_{i_{1}, i_{2}, j_{1,2}}\right|_{U_{\mathbf{i}, \mathbf{j}}} \circ \varphi_{i_{0}, i_{1}, j_{0,1}}\right|_{U_{\mathbf{i}, \mathbf{j}}}
$$

Here and subsequently, to minimize notational clutter we generally leave as implicit restrictions of identity maps to the appropriate open subsets.

We now check that the $\rho^{\prime}$ we have constructed is a cocycle. Given $\mathbf{i}=\left(i_{0}, i_{1}\right.$, $\left.i_{2}, i_{3}\right)$ and $\mathbf{j}=\left(j_{m, n}\right)_{0 \leq m<n \leq 3}$, denote by $\mathbf{j} \ell$ the triple of $j_{m, n}$ with $m, n \neq \ell$. To check that $\left(d \rho^{\prime}\right)_{\mathbf{i}, \mathbf{j}}=0$, we want to see that

$$
\left.\rho_{i_{0}, i_{1}, i_{2}, \mathbf{j}_{3}}^{\prime}\right|_{U_{\mathbf{i}, \mathbf{j}}}-\left.\rho_{i_{0}, i_{1}, i_{3}, \mathbf{j}_{2}}^{\prime}\right|_{U_{\mathbf{i}, \mathbf{j}}}+\left.\rho_{i_{0}, i_{2}, i_{3}, \mathbf{j}_{1}}^{\prime}\right|_{U_{\mathbf{i}, \mathbf{j}}}-\left.\rho_{i_{1}, i_{2}, i_{3}, \mathbf{j}_{0}}^{\prime}\right|_{U_{\mathbf{i}, \mathbf{j}}}=0,
$$


or equivalently,

$$
\left.\left.\rho_{i_{0}, i_{2}, i_{3}, \mathbf{j}_{1}}^{\prime}\right|_{U_{\mathbf{i}, \mathbf{j}}} \circ \rho_{i_{0}, i_{1}, i_{2}, \mathbf{j} 3}^{\prime}\right|_{U_{\mathbf{i}, \mathbf{j}}}=\left.\left.\rho_{i_{0}, i_{1}, i_{3}, \mathbf{j}_{2}}^{\prime}\right|_{U_{\mathbf{i}, \mathbf{j}}} \circ \rho_{i_{1}, i_{2}, i_{3}, \mathbf{j}_{0}}^{\prime}\right|_{U_{\mathbf{i}, \mathbf{j}}}
$$

By Corollary 2.36 (iii) this is equivalent to checking that

$$
\operatorname{id}_{\rho_{i_{0}}}+\left(\left.\left.\rho_{i_{0}, i_{1}, i_{2}, \mathbf{j}_{3}}^{\prime}\right|_{U_{\mathbf{i}, \mathbf{j}}} \circ \rho_{i_{0}, i_{2}, i_{3}, \mathbf{j}_{1}}^{\prime}\right|_{U_{\mathbf{i}, \mathbf{j}}}\right)=\operatorname{id}_{\rho_{i_{0}}}+\left(\left.\left.\rho_{i_{0}, i_{1}, i_{3}, \mathbf{j}_{2}}^{\prime}\right|_{U_{\mathbf{i}, \mathbf{j}}} \circ \rho_{i_{1}, i_{2}, i_{3}, \mathbf{j}_{0}}^{\prime}\right|_{U_{\mathbf{i}, \mathbf{j}}}\right) \text {. }
$$

But using Corollary 3.6 and Corollary 2.36 (iii) we have

$$
\operatorname{id}_{\rho_{i_{0}}}+\left(\left.\left.\rho_{i_{0}, i_{1}, i_{2}, \mathbf{j}_{3}}^{\prime}\right|_{U_{\mathbf{i}, \mathbf{j}}} \circ \rho_{i_{0}, i_{2}, i_{3}, \mathbf{j}_{1}}^{\prime}\right|_{U_{\mathbf{i}, \mathbf{j}}}\right)=\left.\left.\left(\operatorname{id}_{\rho_{i_{0}}}+\rho_{i_{0}, i_{1}, i_{2}, \mathbf{j}_{3}}^{\prime}\right)\right|_{U_{\mathbf{i}, \mathbf{j}}} \circ\left(\operatorname{id}_{\rho_{i_{0}}}+\rho_{i_{0}, i_{2}, i_{3}, \mathbf{j}_{1}}^{\prime}\right)\right|_{U_{\mathbf{i}, \mathbf{j}}}
$$

and using also Corollary 2.36 (iv) we find

$$
\begin{aligned}
& \operatorname{id}_{\rho_{i_{0}}}+\left(\left.\left.\rho_{i_{0}, i_{1}, i_{3}, \mathbf{j}_{2}}^{\prime}\right|_{U_{\mathbf{i}, \mathbf{j}}} \circ \rho_{i_{1}, i_{2}, i_{3}, \mathbf{j}_{0}}^{\prime}\right|_{U_{\mathbf{i}, \mathbf{j}}}\right) \\
& \quad=\left(\operatorname{id}_{\rho_{i_{0}}}+\left.\rho_{i_{0}, i_{1}, i_{3}, \mathbf{j}_{2}}^{\prime}\right|_{U_{\mathbf{i}, \mathbf{j}}}\right) \circ\left(\operatorname{id}_{\rho_{i_{0}}}+\left.\rho_{i_{1}, i_{2}, i_{3}, \mathbf{j}_{0}}^{\prime}\right|_{U_{\mathbf{i}, \mathbf{j}}}\right) \\
& \quad=\left.\left(\operatorname{id}_{\rho_{i_{0}}}+\rho_{i_{0}, i_{1}, i_{3}, \mathbf{j}_{2}}^{\prime}\right)\right|_{U_{\mathbf{i}, \mathbf{j}}} \circ\left(\left.\left.\left.\varphi_{i_{0}, i_{1}, j_{0,1}}^{-1}\right|_{U_{\mathbf{i}, \mathbf{j}}} \circ\left(\operatorname{id}_{\rho_{i_{1}}}+\rho_{i_{1}, i_{2}, i_{3}, \mathbf{j}_{0}}^{\prime}\right)\right|_{U_{\mathbf{i}, \mathbf{j}}} \circ \varphi_{i_{0}, i_{1}, j_{0,1}}\right|_{U_{\mathbf{i}, \mathbf{j}}}\right),
\end{aligned}
$$

and finally expanding both sides in terms of the various $\varphi_{\mathbf{i}, \mathbf{j}}$ we obtain the desired identity.

A similar argument shows that our constructed map to $H^{2}\left(X, \mathcal{A}_{I}\right)$ is welldefined: first, if we modify our $\varphi_{i_{0}, i_{1}, j}$ by automorphisms $\gamma_{i_{0}, i_{1}, j}$ of the $\left.\rho_{i_{0}}\right|_{U_{i_{0}, i_{1}, j}}$, we change the resulting cocycle by a coboundary. Indeed, if $\delta_{i_{0}, i_{1}, j} \in \mathcal{A}_{I}\left(U_{i_{0}, i_{1}, j}\right)$ is determined by $\mathrm{id}_{\rho_{i_{0}}}+\delta_{i_{0}, i_{1}, j}=\gamma_{i_{0}, i_{1}, j}$ for all $i_{0}, i_{1}, j$, and if we fix triples $\mathbf{i}=$ $\left(i_{0}, i_{1}, i_{2}\right), \mathbf{j}=\left(j_{0,1}, j_{0,2}, j_{1,2}\right)$, we assert that precomposing the $\varphi_{i_{0}, i_{1}, j}$ by $\gamma_{i_{0}, i_{1}, j}$ has the effect of modifying $\rho_{\mathbf{i}, \mathbf{j}}^{\prime}$ by $\left.\delta_{i_{0}, i_{1}, j_{0,1}}\right|_{U_{\mathbf{i}, \mathbf{j}}}-\left.\delta_{i_{0}, i_{2}, j_{0,2}}\right|_{U_{\mathbf{i}, \mathbf{j}}}+\left.\delta_{i_{1}, i_{2}, j_{1,2}}\right|_{U_{\mathbf{i}, \mathbf{j}}}$. To check this, using Corollary 2.36 (iv) and Corollary 3.6 we see that

$$
\operatorname{id}_{\rho_{i_{0}}}+\left.\delta_{i_{1}, i_{2}, j_{1,2}}\right|_{U_{\mathbf{i}, \mathbf{j}}}=\left.\left.\left.\varphi_{i_{0}, i_{1}, j_{0,1}}^{-1}\right|_{U_{\mathbf{i}, \mathbf{j}}} \circ \gamma_{i_{1}, i_{2}, j_{1,2}}\right|_{U_{\mathbf{i}, \mathbf{j}}} \circ \varphi_{i_{0}, i_{1}, j_{0,1}}\right|_{U_{\mathbf{i}, \mathbf{j}}}
$$

and

$$
\operatorname{id}_{\rho_{i_{0}}}+\left.\left(-\delta_{i_{0}, i_{2}, j_{0,2}}\right)\right|_{U_{\mathbf{i}, \mathbf{j}}}=\left.\left.\left.\varphi_{i_{2}, i_{0}, j_{0,2}}\right|_{U_{\mathbf{i}, \mathbf{j}}} \circ \gamma_{i_{2}, i_{0}, j_{0,2}}\right|_{U_{\mathbf{i}, \mathbf{j}}} \circ \varphi_{i_{2}, i_{0}, j_{0,2}}^{-1}\right|_{U_{\mathbf{i}, \mathbf{j}}},
$$

and we then obtain the required identity by expanding

$$
\begin{aligned}
& \operatorname{id}_{\rho_{i_{0}}}+\left(\rho_{\mathbf{i}, \mathbf{j}}^{\prime}+\left.\delta_{i_{0}, i_{1}, j_{0,1}}\right|_{U_{\mathbf{i}, \mathbf{j}}}-\left.\delta_{i_{0}, i_{2}, j_{0,2}}\right|_{U_{\mathbf{i}, \mathbf{j}}}+\left.\delta_{i_{1}, i_{2}, j_{1,2}}\right|_{U_{\mathbf{i}, \mathbf{j}}}\right) \\
& \quad=\mathrm{id}_{\rho_{i_{0}}}+\left(\left.\left.\left(-\left.\delta_{i_{0}, i_{2}, j_{0,2}}\right|_{U_{\mathbf{i}, \mathbf{j}}}\right) \circ \rho_{\mathbf{i}, \mathbf{j}}^{\prime} \circ \delta_{i_{1}, i_{2}, j_{1,2}}\right|_{U_{\mathbf{i}, \mathbf{j}}} \circ \delta_{i_{0}, i_{1}, j_{0,1}}\right|_{U_{\mathbf{i}, \mathbf{j}}}\right) .
\end{aligned}
$$

Next, if we replace our choices of $\left(\rho_{i}, \varphi_{i}\right)$ by different but isomorphic choices, we simply fix isomorphisms $\alpha_{i}$ on each $U_{i}$, and replace each $\varphi_{i_{0}, i_{1}, j}$ by $\left.\alpha_{i_{1}}^{-1}\right|_{U_{i_{0}, i_{1}, j}} \circ$ $\left.\varphi_{i_{0}, i_{1}, j} \circ \alpha_{i_{0}}\right|_{U_{i_{0}, i_{1}, j}}$, and using Corollary 2.36 once more we verify that $\rho^{\prime}$ remains unchanged. Finally, given any two choices of the $\left(\rho_{i}, \varphi_{i}\right)$ on any two covers $\left\{U_{i}\right\}$, if we take a common refinement and refine further if necessary so that our choices 
are isomorphic on each $\left\{U_{i}\right\}$, we see that our choices differ by a coboundary, so our map is well defined.

It then follows that we have the desired functoriality, as well: if we carry out the construction for $\rho$, using Propositions 3.4 (i) and 2.6 (iv) and the functoriality of addition of automorphisms in Corollary 2.30 we see that pushforward to $B^{\prime}$ commutes with each step of the construction, so that the image of $\left.\rho\right|_{B^{\prime}}$ is $\left.\rho^{\prime}\right|_{J}$, as desired.

We next check exactness at $\Gamma\left(X, \mathcal{T}_{\eta}, A^{\prime}\right)$ : the kernel of the constructed map consists precisely of the $\rho$ for which, after possible refinement of the cover, there exist a collection of $\rho_{i_{0}, i_{1}, j}^{\prime \prime} \in \mathcal{A}_{I}\left(U_{i_{0}, i_{1}, j}\right)$ such that $\rho^{\prime}=d \rho^{\prime \prime}$. Given any collection of $\rho^{\prime \prime}$, one checks that if we set $\varphi_{i_{0}, i_{1}, j}^{\prime}=\varphi_{i_{0}, i_{1}, j} \circ\left(\operatorname{id}_{\rho_{i_{0}}}-\rho_{i_{0}, i_{1}, j}^{\prime \prime}\right)$, then $\rho^{\prime}=d \rho^{\prime \prime}$ is equivalent to the $\varphi^{\prime}$ satisfying the cocycle condition, and the existence of such $\varphi^{\prime}$ is in turn equivalent to being able to glue the $\rho_{i_{0}}$ compatibly to obtain an element of $T_{\eta, A^{\prime}}$ inducing $\rho$. Since the $\rho^{\prime \prime}$ can be recovered from the $\varphi^{\prime}$, this proves exactness.

Finally, we check the additivity and linearity assertions. For additivity, we use the additivity of pullbacks given in Corollary 3.6: suppose we have $\rho^{1} \in$ $\Gamma\left(X, \mathcal{T}_{\eta}, A^{\prime}\right)$ and $\rho^{2} \in \Gamma\left(X, \mathcal{T}_{I}\right)$, which we have chosen to be represented by $\left\{\rho_{i}^{1}\right\}$ and $\left\{\rho_{i}^{2}\right\}$ respectively on a common cover $\left\{U_{i}\right\}$, with isomorphisms $\left\{\varphi_{i_{0}, i_{1}, j}^{1}\right\}$ and $\left\{\varphi_{i_{0}, i_{1}, j}^{2}\right\}$ on common refinements $U_{i_{0}, i_{1}, j}$. The resulting cocycles $\rho^{1^{\prime}}$ and $\rho^{2^{\prime}}$ are characterized by the condition that on each $U_{\mathbf{i}, \mathbf{j}}$, for $m=1,2$ we have

$$
\rho^{m \prime}{ }_{i_{0}, i_{1}, i_{2}, \mathbf{j}}+\mathrm{id}_{\rho_{i_{0}}^{m}}=\varphi_{i_{2}, i_{0}, j_{0,2}}^{m} \circ \varphi_{i_{1}, i_{2}, j_{1,2}}^{m} \circ \varphi_{i_{0}, i_{1}, j_{0,1}}^{m} .
$$

We can then represent $\rho^{1}+\rho^{2}$ by $\left\{\rho_{i}^{1}+\rho_{i}^{2}\right\}$ on $\left\{U_{i}\right\}$, and choose our isomorphisms on $U_{i_{0}, i_{1}, j}$ to be given by $\varphi_{i_{0}, i_{1}, j}^{1}+\varphi_{i_{0}, i_{1}, j}^{2}$. The desired assertion then comes down to checking that

$$
\begin{aligned}
& \left(\operatorname{id}_{\left.\rho_{i_{0}}^{1}\right|_{U_{\mathbf{i}, \mathbf{j}}}}+\rho^{1^{\prime}{ }_{i_{0}, i_{1}, i_{2}, \mathbf{j}}}\right)+\left(\operatorname{id}_{\left.\rho_{i_{0}}^{2}\right|_{U_{\mathbf{i}, \mathbf{j}}}}+\rho^{2}{ }_{i_{0}, i_{1}, i_{2}, \mathbf{j}}^{\prime}\right) \\
& =\operatorname{id}_{\left.\rho_{i_{0}}^{1}\right|_{U_{\mathbf{i}, \mathbf{j}}}+\left.\rho_{i_{0}}^{2}\right|_{U_{\mathbf{i}, \mathbf{j}}}}+\left(\rho^{1^{\prime}{ }_{i_{0}, i_{1}, i_{2}, \mathbf{j}}}+\rho^{2}{ }_{i_{0}, i_{1}, i_{2}, \mathbf{j}}^{\prime}\right) .
\end{aligned}
$$

But this is Corollary 2.36 (vi).

Lastly, in the case that $A^{\prime}=k[I]$, our map is also $k$-linear: one need only verify that it commutes with scalar multiplication, which is easily checked directly: we may represent $m_{\lambda *} \rho$ by $m_{\lambda *} \rho_{i}$ on each $U_{i}$, with isomorphisms $m_{\lambda *} \varphi_{i_{0}, i_{1}, j}$, and we get the desired identity from the fact that $m_{\lambda *}$ commutes with addition of automorphisms and composition of morphisms.

We are now ready to prove our first main theorem.

Proof of Theorem 1.1. We first address the exact sequence for $T_{\mathcal{S}}$. The second map is obtained from the sheafification map, and is therefore automatically $k$-linear, with its kernel consisting precisely of deformations which are locally trivial. The first map is constructed by considering such locally trivial deformations: given a Čech 
1-cocycle $\rho$ of $\mathcal{A}$ in terms of an open cover $\left\{U_{i}\right\}$, on each $U_{i}$ we can take the trivial deformation, and by the stack condition for $\mathcal{S}$ over $\operatorname{Zar}(X)$, we can use $\rho$ to glue the deformations to obtain an $\eta_{\rho} \in T_{\mathcal{S}}$, which moreover will be trivial if and only if $\rho$ can be simultaneously trivialized by automorphisms of the $U_{i}$; i.e., if and only if $\rho$ is a coboundary. This gives the first inclusion, and it is clear that the image is precisely the set of locally trivial deformations, so we obtain exactness at $T_{\mathcal{S}}$. One also checks easily that the inclusion map is $k$-linear using the properties given in Proposition 3.4 and the fact from Proposition 3.5 and Corollary 3.6 that $+_{k[\epsilon],(\epsilon)}$ is a functor, and we can use it to add pullback maps.

The last map is obtained from Proposition 3.12 in the case $V=(\epsilon)$, completing the desired exact sequence.

We now consider obstructions. We therefore fix a small extension $A^{\prime} \rightarrow A$ in $\operatorname{Art}(\Lambda, k)$ with kernel $I$, and an object $\eta \in \mathcal{S}_{(X, A)}$. By definition, we get an element $\mathrm{ob}_{\eta, A^{\prime}}^{1} \in H^{0}(X, \mathcal{O} b) \otimes I$, which is zero if and only if $\eta$ can be extended to $A^{\prime}$ locally. If $o b_{\eta, A^{\prime}}^{1} \neq 0$, we set this as our obstruction.

If it is zero, there exists some cover $\left\{U_{i}\right\}$ of $X$ and liftings $\eta_{i}^{\prime}$ of $\left.\eta\right|_{U_{i}}$ from $A$ to $A^{\prime}$. The next question is whether these liftings can be chosen so that they are isomorphic on the $U_{i_{0}, i_{1}}$. By Corollary 2.30 and Proposition 2.40, the choices of the $\eta_{i}^{\prime}$ together with given pullback maps $\left.\eta\right|_{U_{i}} \rightarrow \eta_{i}^{\prime}$ over $A^{\prime} \rightarrow A$ (up to simultaneous isomorphism) are torsors under $T_{\left.\mathcal{S}_{\left(U_{i},-\right.}\right)} \otimes I$, so if we restrict to the $U_{i_{0}, i_{1}}$, taking differences of $\eta_{i_{0}}^{\prime}$ and $\eta_{i_{1}}^{\prime}$ give elements $\eta_{i_{0}, i_{1}}^{\prime} \in T_{\mathcal{S}_{\left(U_{\left.i_{0}, i_{1},{ }^{\prime}\right)}\right.}} \otimes I$. Moreover, by Lemma 3.8 and general properties of torsors we see that given $i_{0}, i_{1}, i_{2}$ we have the cocycle condition

$$
\left.\eta_{i_{0}, i_{1}}^{\prime}\right|_{U_{i_{0}, i_{1}, i_{2}}}+\left.\eta_{i_{1}, i_{2}}^{\prime}\right|_{U_{i_{0}, i_{1}, i_{2}}}+\left.\eta_{i_{2}, i_{0}}^{\prime}\right|_{U_{i_{0}, i_{1}, i_{2}}}=0
$$

so we obtain an element $\mathrm{ob}_{\eta, A^{\prime}}^{2} \in H^{1}(X, \mathcal{T}) \otimes I$. Furthermore, this element vanishes if and only if the choices of lifts $\eta_{i}^{\prime}$ can be modified simultaneously so that they are all isomorphic on every $U_{i_{0}, i_{1}}$, giving us an element of $\Gamma\left(X, \mathcal{T}_{\eta, A^{\prime}}\right)$. Thus, if $\mathrm{ob}_{\eta, A^{\prime}}^{2} \neq 0$, we use it as our obstruction. We observe that this is independent of choices: modifying our choices of the $\eta_{i}^{\prime}$ changes the constructed cocycle by a coboundary, while different choices of the cover $\left\{U_{i}\right\}$ may be compared by restriction to a common refinement. In particular, $\mathrm{ob}_{\eta, A^{\prime}}^{2}=0$ if and only if $\Gamma\left(X, \mathcal{T}_{\eta, A^{\prime}}\right)$ is non-empty.

Finally, if $\mathrm{ob}_{\eta, A^{\prime}}^{2}=0$, by choosing lifts $\eta_{i}^{\prime}$ which agree on $U_{i_{0}, i_{1}}$ we obtain an element of $\Gamma\left(X, \mathcal{T}_{\eta, A^{\prime}}\right)$, and by Proposition 3.12 above and using the isomorphism $\mathcal{A}_{I} \stackrel{\sim}{\rightarrow} \mathcal{A} \otimes_{k} I$ of Proposition 2.40, we obtain an element of $H^{2}(X, \mathcal{A}) \otimes I$. We then set $\mathrm{ob}_{\eta, A^{\prime}}^{3}$ to be its image in

$$
\left(H^{2}(X, \mathcal{A}) / H^{0}(X, \mathcal{T})\right) \otimes I=\left(H^{2}(X, \mathcal{A}) \otimes I\right) /\left(H^{0}(X, \mathcal{T}) \otimes I\right) .
$$

Moreover, because $\Gamma\left(X, \mathcal{T}_{\eta, A^{\prime}}\right)$ is a torsor over $H^{0}\left(X, \mathcal{T}_{I}\right)=H^{0}(X, \mathcal{T}) \otimes I$, it follows from the additivity in Proposition 3.12 that $\mathrm{ob}_{\eta, A^{\prime}}^{3}$ is independent of the 
choice of element of $\Gamma\left(X, \mathcal{T}_{\eta}, A^{\prime}\right)$. It thus follows from the exactness in Proposition 3.12 that if $T_{\eta, A^{\prime}}$ is non-empty, we have $\mathrm{ob}_{\eta, A^{\prime}}^{3}=0$. Conversely, if $\mathrm{ob}_{\eta, A^{\prime}}^{3}=0$, we have an element of $\mu \in \Gamma\left(X, \mathcal{T}_{\eta}, A^{\prime}\right)$ whose image inside $H^{2}\left(X, \mathcal{A}_{I}\right)$ agrees with the image of some $\rho \in H^{0}(X, \mathcal{T}) \otimes I=H^{0}\left(X, \mathcal{T}_{I}\right)$. Thus, by the additivity and exactness in Proposition 3.12 we see that $\rho-\mu$ maps to 0 in $H^{2}\left(X, \mathcal{A}_{I}\right)$ and hence is the image of an element of $T_{\eta, A^{\prime}}$, meaning that an $\eta^{\prime}$ lifting $\eta$ exists, as desired.

It remains only to check that the obstruction theory we have constructed is functorial, and this is straightforward: for $\mathrm{ob}^{1}$ this is part of the definition of a local obstruction sheaf; for $\mathrm{ob}^{2}$ it follows from the functoriality in Corollary 2.30 and Propositions 3.4 (i) and 2.40; and for $\mathrm{ob}^{3}$ we use the functoriality in Propositions 3.12 and 2.40 .

Remark 3.13. We observe that in fact in the proof of Theorem 1.1 we did not use the existence of a local obstruction sheaf, but merely of a single vector space which measures the local obstruction to extending a given global object. However, whenever one has such a space one expects to obtain such spaces under restriction to every $U \subseteq X$, and therefore to obtain a local obstruction sheaf, so the sheaf terminology seems more natural.

We are now able to say quite a bit in the context of Schlessinger's theory of representability and hulls as well, proving our second main theorem.

Proof of Theorem 1.2. Putting together Proposition 2.10, Proposition 2.12, Theorem 1.9 and Theorem 1.1, we obtain everything except the last part of the desired statement. Noting that from the first exact sequence of Theorem 1.1, the dimension of the third obstruction space $H^{2}(X, \mathcal{A}) / H^{0}(X, \mathcal{T})$ is given by $\operatorname{dim} T_{\mathcal{S}}+$ $h^{2}(X, \mathcal{A})-h^{1}(X, \mathcal{A})-h^{0}(X, \mathcal{T})$, the only ingredient still missing is the below theorem, entirely in the realm of classical deformation theory.

Theorem 3.14. Let $F$ be a deformation functor satisfying (H3), so that it has a hull $(R, \xi)$. Suppose that $F$ has a successive obstruction theory taking values in finite-dimensional spaces $V_{1}, \ldots, V_{m}$. Then we have:

$$
\operatorname{dim} T_{F}-\sum_{i} \operatorname{dim} V_{i} \leq \operatorname{dim} R-\operatorname{dim} \Lambda \leq \operatorname{dim} T_{F}
$$

and if the first inequality is an equality and $\Lambda$ is regular, then $R$ is a local complete intersection ring. If further $\operatorname{dim} V_{i}=0$ for all $i$, then $R$ is smooth over $\Lambda$.

The theorem is essentially due to Mori, and largely follows the argument presented in [10, Proposition 2.A.11] and [5, Lemma 6.3.3]. We include the argument here partly for convenience, and partly because the use of successive obstruction spaces make our statement slightly more general, although the adjustment to the argument is straightforward. 
We use a simple lemma to reduce to the prorepresentable case:

Lemma 3.15. Suppose that $F_{1}, F_{2}$ are functors $\operatorname{Art}(\Lambda, k) \rightarrow$ Set, and we have a morphism $f: F_{1} \rightarrow F_{2}$ which is formally smooth, and a successive obstruction theory for $F_{2}$ taking values in $V_{1}, \ldots, V_{m}$. Then $f$ induces a successive obstruction theory for $F_{1}$ taking values in $V_{1}, \ldots, V_{m}$.

Proof. Given $\eta \in F_{1}(A)$, and a small extension $A^{\prime} \rightarrow A$, we can define the obstruction $\mathrm{ob}_{\eta, A^{\prime}}$ to be simply ob $f(\eta), A^{\prime}$. The smoothness of $f$ then implies that $\eta$ can be lifted to $A^{\prime}$ if and only if $f(\eta)$ can be lifted to $A^{\prime}$, so the main conditions for an obstruction theory are satisfied, and it remains only to check functoriality, which follows from functoriality of the obstruction theory given for $F_{2}$ together with the required functoriality of $f$.

Proof of Theorem 3.14. By the definition of a hull $\left.h_{R}\right|_{\operatorname{Art}(\Lambda, k)} \stackrel{\xi}{\rightarrow} F$ is formally smooth, and gives an isomorphism of tangent spaces. By the lemma, we have a successive obstruction theory taking values in the same $V_{i}$ for $\left.h_{R}\right|_{\operatorname{Art}(\Lambda, k)}$ as well, so it is enough to prove the theorem in the case that $F$ is prorepresentable.

In this case, we work explicitly: if we write $d=\operatorname{dim} T_{F}$, Schlessinger's construction of $R$ in the [18, proof of 2.11] is as a quotient of $B:=\Lambda\left[\left[t_{1}, \ldots, t_{d}\right]\right]$ by some ideal $J$, so to prove the theorem, it is enough to show that the number of generators of $J$ is bounded above by $\sum_{i} \operatorname{dim} V_{i}$.

By the Artin-Rees lemma, $J \cap \mathfrak{m}_{B}^{n} \subseteq J \mathfrak{m}_{B}$ for some $n$. We now set $A^{(0)}=$ $\Lambda\left[\left[t_{1}, \ldots, t_{d}\right]\right] /\left(\mathfrak{m}_{B} J+\mathfrak{m}_{B}^{n}\right)$, and $A=R / \mathfrak{m}_{R}^{n}=\Lambda\left[\left[t_{1}, \ldots, t_{d}\right]\right] /\left(J+\mathfrak{m}_{B}^{n}\right)$, so that we get a small extension

$$
0 \rightarrow I \rightarrow A^{(0)} \rightarrow A \rightarrow 0
$$

with $I=\left(J+\mathfrak{m}_{B}^{n}\right) /\left(\mathfrak{m}_{B} J+\mathfrak{m}_{B}^{n}\right)=J / \mathfrak{m}_{B} J$. From the natural map $R \rightarrow A$ we obtain an object $\xi_{A} \in F(A)$, with an obstruction $\mathrm{ob}_{\xi_{A}, A^{(0)}} \in V_{i} \otimes_{k} I$ to extending $\xi_{A}$ to $A^{(0)}$, for some $i$. If $i=1$, we can write $\mathrm{ob}_{\xi_{A}, A^{(0)}}=\sum_{j=1}^{\operatorname{dim} V_{1}} v_{1, j} \otimes \bar{x}_{1, j}$, where the $v_{1, j}$ form a basis for $V_{1}$, and the $\bar{x}_{j}$ are the images in $I$ of elements $x_{1, j} \in J$. If $i>0$, we declare the $x_{1, j}$ all to be 0 . We then consider the ring $A^{(1)}:=A^{(0)} /\left(x_{1,1}, \ldots, x_{1, \operatorname{dim} V_{1}}\right)$; this surjects onto $A$ with kernel $I^{(1)}$, and we again have an obstruction $\mathrm{ob}_{\xi_{A}, A^{(1)}}$ to extending $\xi_{A}$ to $A^{(1)}$.

If $m=1$, we stop. Otherwise, by the functoriality of the obstruction, we see that $\mathrm{ob}_{\xi_{A}, A^{(1)}} \in V_{i^{\prime}} \otimes_{k} I^{(1)}$ for $i^{\prime}>1$ : indeed, we could only have $i^{\prime}=1$ if we had before $i=1$, in which case the functoriality implies, since we modded out by the $x_{1, j}$, that $\mathrm{ob}_{\xi_{A}, A^{(1)}}=0$, which is only allowed if $m=1$. We thus can write $\mathrm{ob}_{\xi_{A}, A^{(1)}}=\sum_{j=1}^{\operatorname{dim} V_{2}} v_{2, j} \otimes \bar{x}_{2, j}$ as before (again, setting all $x_{2, j}=0$ if $i^{\prime}>2$ ), and set $A^{(2)}=A^{(1)} /\left(x_{2, j}, \ldots, x_{2, \operatorname{dim} V_{2}}\right)$. We repeat this process until we have constructed $A^{\prime \prime}:=A^{(m)}$, which we see immediately is obtained from $A^{\prime}$ by modding out by (at most) $\sum_{i} V_{i}$ elements. 
We note that again by the functoriality of obstructions, we will necessarily have $\mathrm{ob}_{\xi_{A}, A^{\prime \prime}}=0$. Thus, $\xi_{A}$ may be lifted to $A^{\prime \prime}$, and because $F=\left.h_{R}\right|_{\operatorname{Art}(\Lambda, k)}$, this means we can lift the map $R \rightarrow A$ to a map $R \rightarrow A^{\prime \prime}$. We wish to show that this implies

$$
J \subseteq \mathfrak{m}_{B} J+\left(\left\{x_{i, j}\right\}_{i, j}\right)+\mathfrak{m}_{B}^{n},
$$

which is equivalent to the stronger assertion that we have a lifting which commutes with the natural quotient maps from $\Lambda\left[\left[t_{1}, \ldots, t_{d}\right]\right]$ to $R$ and to $A^{\prime \prime}$. Now, if we are given any lifting, we have

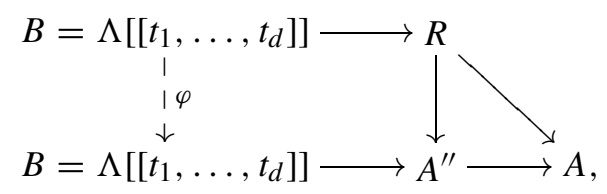

and we can fill in the dashed arrow $\varphi$ to make the diagram commute by choosing appropriate values for $\varphi\left(t_{i}\right), i=1, \ldots, d$. By hypothesis, $\varphi$ commutes with the maps to $A$, so must be the identity modulo $J+\mathfrak{m}_{B}^{n}$. In particular, we conclude that $\varphi$ induces the identity map on $\mathfrak{m}_{B} / \mathfrak{m}_{B}^{2}$, so is an isomorphism, and then that $\varphi^{-1}(J) \subseteq J+\mathfrak{m}_{B}^{n}$, so that $J \subseteq \varphi(J)+\varphi\left(\mathfrak{m}_{B}^{n}\right)=\varphi(J)+\mathfrak{m}_{B}^{n}$. But by commutativity of the maps to $R$ and $A^{\prime \prime}$, we see $\varphi(J) \subseteq \mathfrak{m}_{B} J+\left(\left\{x_{i, j}\right\}_{i, j}\right)+\mathfrak{m}_{B}^{n}$, and putting these together gives (3.1).

Since we had originally $J \cap \mathfrak{m}_{B}^{n} \subseteq \mathfrak{m}_{B} J$, we finally conclude that $J$ is contained in, hence equal to $\mathfrak{m}_{B} J+\left(\left\{x_{i, j}\right\}_{i, j}\right)$. By Nakayama's lemma, we conclude that $J$ is generated by $\left\{x_{i, j}\right\}_{i, j}$, as desired.

Remark 3.16. We observe that Theorem 3.14 only uses a weaker functoriality than what we impose in the definition of an obstruction theory: namely, that the obstruction elements for $A^{\prime} \rightarrow A$ be functorial for restriction to any intermediate small extensions obtained from quotients of $A^{\prime}$. However, in practice it appears that the stronger functoriality is always satisfied, and the stronger functoriality makes our definition compatible with definitions used in other contexts, for instance by Artin ((2.6) of [1]).

\subsection{Special cases and examples}

Specializing to the case of locally unobstructed geometric deformation stacks, we immediately find two special cases of Theorem 1.1 in which we obtain descriptions of tangent and obstruction spaces in terms of standard sheaf cohomology. The first is when we have no non-trivial infinitesimal automorphisms.

Corollary 3.17. Let $\mathcal{S}$ be a geometric deformation stack which is locally unobstructed, and has trivial infinitesimal automorphisms (i.e., $\mathcal{A}=0$ ). Then the presheaf given by $U \mapsto T_{\left.\mathcal{S}_{(U,-}\right)}$ is already a sheaf, and we have $T_{\mathcal{S}}=H^{0}(X, \mathcal{T})$, with obstructions lying in $H^{1}(X, \mathcal{T})$. 
The second case is the situation mentioned in the introduction.

Corollary 3.18. Let $\mathcal{S}$ be a geometric deformation stack which is locally unobstructed, and has locally trivial deformations (i.e., $\mathcal{T}=0$ ). Then we have $T_{\mathcal{S}}=$ $H^{1}(X, \mathcal{A})$, and obstructions lie in $H^{2}(X, \mathcal{A})$.

We remark that there is a situation, less general than that of Theorem 1.1, in which the tangent and obstruction spaces are described as hypercohomology groups of a two-term complex. This situation simultaneously generalizes the two cases above, and is examined in [16].

We next return to the examples examined earlier. We first point out that all examples discussed in Section 2.3 in fact have natural structures of geometric deformation stacks, and that given that each example gives a deformation stack over every open set, checking the geometric deformation stack conditions is a mere formality, because we need only work with the Zariski topology. We will take for granted the various well-known descriptions of automorphism, local deformation, and local obstruction sheaves. However, we will then be able to conclude a number of the tangent and obstruction space descriptions as formal consequences of Theorem 1.1.

Example 3.19. Deformations of sheaves. Given $X_{\Lambda}$ flat over $\Lambda$, and a coherent $\mathcal{O}_{X}$-module $\mathcal{E}$, where $X=\left.X_{\Lambda}\right|_{\text {Spec } k}$, let $\mathcal{G D} e f_{X_{\Lambda}}(\mathcal{E})$ be the associated geometric deformation stack of deformations of $\mathcal{E}$. The automorphism sheaf is then $\mathcal{H} \operatorname{om}(\mathcal{E}, \mathcal{E})$, the local deformation sheaf is $\mathcal{E} x t^{1}(\mathcal{E}, \mathcal{E})$, and $\mathcal{E} x t^{2}(\mathcal{E}, \mathcal{E})$ is a local obstruction sheaf. Indeed, this follows from [11, Proposition 3.1.5 of Chapter IV].

In particular, if $\mathcal{E}$ is locally free, the latter two sheaves are 0 , and by Corollary 3.18 we have that the tangent space is $H^{1}(X, \mathcal{E} n d(\mathcal{E}))$, with obstructions lying in $H^{2}(X, \mathcal{E} n d(\mathcal{E}))$.

Example 3.20. Deformations of schemes. Let $X$ be a scheme over $k$, and $\mathcal{G D} e f(X)$ the associated geometric deformation stack of deformations of $X$. The automorphism sheaf, local deformation sheaf, and obstruction sheaf are described by the Lichtenbaum-Schlessinger $T^{i}$ sheaves on $X$; see [12, Section 2.4 as well as 4.3 .3 and 4.3.4]. We do not describe these sheaves in general, but remark that $T^{0}=$ $\mathcal{H o m}\left(\Omega_{X / k}^{1}, \mathcal{O}_{X}\right)$, and when $X$ is a local complete intersection scheme, we have $T^{2}=0$, and if further $X$ is generically smooth, we have $T^{1}=\mathcal{E} x t^{1}\left(\Omega_{X / k}^{1}, \mathcal{O}_{X}\right)$. The assertion on $T^{2}$ is $[12,3.2 .2]$, and we sketch how to see both assertions, following the notation of loc. cit.

We suppose that $U=\operatorname{Spec} B$ is an affine open subset of $X$, with $B$ realized as the quotient of a polynomial ring $R$ by an ideal $I$. The $T^{i}$ on $U$ are defined as the cohomology of $\operatorname{Hom}\left(L^{\bullet}, B\right)$, where $L^{\bullet}$ is a complex of $B$-modules constructed as follows. Let $F$ be a free $R$-module, with a surjection $j: F \rightarrow I$, with kernel $J$. Let $J_{0}$ be the submodule (a priori of $F$, but in fact of $J$ ) generated by all elements of the form $j\left(f_{1}\right) f_{2}-j\left(f_{2}\right) f_{1}$ for $f_{1}, f_{2} \in F$. The cotangent complex is then defined as

$$
J / J_{0} \rightarrow F / J_{0} \otimes_{R} B \rightarrow \Omega_{R / k}^{1} \otimes_{R} B .
$$


Note that $J / J_{0}$ can be given a $B$-module structure, and that $F / J_{0} \otimes_{R} B=F \otimes_{R}$ $B=F / I F$ (see [9, Section 3.1]). By [8, Proposition II.8.4A], the map $I / I^{2} \rightarrow$ $\Omega_{R / k}^{1} \otimes_{R} B$ and hence our map $F / J_{0} \otimes_{R} B \rightarrow \Omega_{R / k}^{1} \otimes_{R} B$ has cokernel $\Omega_{B / k}^{1}$, giving the desired description of $T^{0}$ (of course, one can also compute the infinitesimal automorphisms directly).

In the case that $X$ is a local complete intersection scheme, we suppose we have chosen $U$ small enough that it may be realized as a complete intersection inside of Spec $R$. Now, the claim is that in this case, the complex $L^{\bullet}$ in fact consists of two terms: $I / I^{2} \rightarrow \Omega_{R / k}^{1} \otimes_{R} B$. We choose $F$ to be a free module generated by a minimal set of generators in $I$, which necessarily form an $R$-sequence. We therefore need to check that $J_{0}=J$, and $F / I F=I / I^{2}$. The first equality follows from exactness of the Koszul complex for the generators of $I$ (see [13, Theorem 16.5(i)]), while the second follows from [8, Theorem II.8.21A(e)].

We then have that $T^{2}$ is the second cohomology of a two-term complex, and hence equal to 0 , as asserted. Furthermore, if $X$ is generically smooth, is it also necessarily locally integral. The complex $I / I^{2} \rightarrow \Omega_{R / k}^{1} \otimes_{R} B$ is generically injective by [8, Theorem II.8.17]. But since both terms in the complex are locally free, the map is in fact injective, and therefore gives a locally free resolution of $\Omega_{B / k}^{1}$, so we conclude that $T^{1}$ computes $\operatorname{Ext}^{1}\left(\Omega_{B / k}^{1}, B\right)$, as desired. See also [20, Theorem 4.4] for an exposition treating the global case.

Finally, if further $X$ is smooth, then also $T^{1}=0$, and $T^{0}=T_{X}$, the tangent sheaf of $X$. We thus get from Corollary 3.18 that the tangent space is $H^{1}\left(X, T_{X}\right)$, with obstructions in $H^{2}\left(X, T_{X}\right)$.

Example 3.21. Deformations of quotient sheaves. Given $\mathcal{E}_{\Lambda}$ coherent on some $X_{\Lambda}$, write $\mathcal{E}$ and $X$ for the restrictions to Spec $k$. Given also a quotient $\mathcal{F}$ of $\mathcal{E}$, let $\mathcal{G} \mathcal{D} e f_{X_{\Lambda}, \mathcal{E}_{\Lambda}}(\mathcal{F})$ be the associated geometric deformation stack of deformations of $\mathcal{F}$ as a quotient of $\mathcal{E}$. Assume further that $\mathcal{E}_{\Lambda}$ is flat over $\Lambda$. Write $\mathcal{G}=\operatorname{ker}(\mathcal{E} \rightarrow \mathcal{F})$ Then the automorphism sheaf is 0 , the local deformation sheaf is given by $\mathcal{H} \operatorname{om}(\mathcal{G}, \mathcal{F})$, and we can take $\mathcal{E} x t^{1}(\mathcal{G}, \mathcal{F})$ as a local obstruction sheaf. See for instance Olsson-Starr ([14, Lemma 2.5] and the subsequent discussion there, noting that under the flatness hypothesis on $\mathcal{E}_{\Lambda}$, the first obstruction discussed there always vanishes).

In particular, if $X_{\Lambda}$ is flat over $\Lambda$, and $\mathcal{E}$ and $\mathcal{F}$ (and therefore also $\mathcal{G}$ ) are locally free, then the local obstruction sheaf is 0 , and we have from Corollary 3.17 that the tangent space is $H^{0}(X, \mathcal{H o m}(\mathcal{G}, \mathcal{F}))$, with obstructions lying in $H^{1}(X, \mathcal{H o m}(\mathcal{G}, \mathcal{F}))$.

Example 3.22. Deformations of subschemes. Let $X_{\Lambda}$ be a scheme flat over $\Lambda$, and $X$ its restriction to Spec $k$. Given $Z \subseteq X$ a closed subscheme with ideal sheaf $\mathcal{I}_{Z}$, let $\mathcal{G D} e f_{X_{\Lambda}}(Z)$ be the associated geometric deformation stack of deformations of $Z$ inside $X$. Then it follows directly from the above case of quotient sheaves that the automorphism sheaf is 0 , the local deformation sheaf is $\mathcal{H o m}_{X}\left(\mathscr{I}_{Z}, \mathcal{O}_{Z}\right)=$ $\mathcal{H}_{\text {om }}\left(\mathscr{I}_{Z} / \mathscr{I}_{Z}^{2}, \mathcal{O}_{Z}\right)$, and the local obstructions lie in $\mathcal{E}_{x} t_{X}^{1}\left(\mathscr{I}_{Z}, \mathcal{O}_{Z}\right)$.

In particular, if $Z$ is a local complete intersection inside $X$, in the sense that it is locally cut out by regular sequences, then the local deformation sheaf is the 
normal bundle $\mathcal{N}_{Z / X}$, and we claim that there are no local obstructions. Indeed, this may be shown directly by observing that any liftings of local equations for $Z$ inside $X$ will yield a local deformation of $Z$; see for instance [20], Lemma 2.7 and the preceding discussion.

Thus, from Corollary 3.17 we see that the tangent space is $H^{0}\left(Z, \mathcal{N}_{Z / X}\right)$ and the obstructions lie in $H^{1}\left(Z, \mathcal{N}_{Z / X}\right)$.

Example 3.23. Deformations of morphisms. Given $X_{\Lambda}, Y_{\Lambda}$ locally of finite type over $\Lambda$, with $X_{\Lambda}$ flat and $Y_{\Lambda}$ separated, and $X$ and $Y$ the respective restrictions to Spec $k$, suppose $f: X \rightarrow Y$ is a morphism, and let $\mathcal{G} \mathcal{D} e f_{X_{\Lambda}, Y_{\Lambda}}(f)$ be the geometric deformation stack of deformations of $f$. Then from the previous example we see that the automorphism sheaf is trivial, the local deformation sheaf is $\mathcal{H} o m\left(f^{*} \Omega_{Y / k}^{1}, \mathcal{O}_{X}\right)$, and local obstructions lie in $\mathcal{E}_{x} t_{X \times_{k} Y}^{1}\left(\mathscr{I}_{\Gamma(f)}, \mathcal{O}_{\Gamma(f)}\right)$.

In particular, if $Y$ is smooth, then $\Gamma(f)$ is a local complete intersection, so we have by the previous example and Corollary 3.17 that the tangent space is $H^{0}\left(X, f^{*} T_{Y}\right)$, with obstructions in $H^{1}\left(X, f^{*} T_{Y}\right)$.

Example 3.24. Deformations of connections. Suppose we have a scheme $X_{\Lambda}$ smooth over $\Lambda$, and a locally free $\mathcal{O}_{X_{\Lambda}}$-module $\mathcal{E}_{\Lambda}$, and write $X$ and $\mathcal{E}$ for the restrictions to Spec $k$. Given also a connection $\nabla$ on $\mathcal{E}$, let $\mathcal{G} \mathcal{D} e f_{X_{\Lambda}, \mathcal{E}_{\Lambda}}(\nabla)$ be the geometric deformation stack of deformations of $\nabla$. Then the automorphism sheaf is 0 by definition, the local deformation sheaf is $\mathcal{H o m}\left(\mathcal{E}, \mathcal{E} \otimes \Omega_{X / k}^{1}\right)$, and local obstructions vanish. Indeed, both the last two statements follow from the fact that $\mathcal{E}$ is locally free, so that connections may be expressed locally explicitly in terms of matrices with coefficients in $\Omega_{X / k}^{1}$. By Corollary 3.17, we find that the tangent space is $H^{0}\left(X, \mathcal{E} n d(\mathcal{E}) \otimes \Omega_{X / k}^{1}\right)$, with obstructions lying in $H^{1}\left(X, \mathcal{E} n d(\mathcal{E}) \otimes \Omega_{X / k}^{1}\right)$.

We note as a consequence of Example 3.20 a non-trivial example of a deformation stack with trivial automorphisms and therefore satisfying (H4):

Corollary 3.25. Suppose that $X / k$ is a scheme with $\operatorname{Hom}\left(\Omega_{X / k}^{1}, \mathcal{O}_{X}\right)=0$. Then the deformation stack $\operatorname{Def}_{X}$ has trivial automorphisms, and therefore satisfies Schlessinger's (H4).

Proof. By Example 3.20, we see that the first-order infinitesimal automorphisms vanish, and the remaining assertions follow from Corollary 2.41.

Example 3.26. Suppose $X$ is a smooth, proper curve of genus at least 2. Then Def $_{X}$ has trivial automorphisms, and satisfies Schlessinger's (H4).

Although some deformation problems, such as those involving two or more schemes, cannot be expressed as a geometric deformation stack in a natural way, geometric deformation stacks are by no means limited to the examples above. Indeed, any deformation problem which can be naturally expressed as occurring over a single scheme should form a geometric deformation stack, including deformations of a pointed curve, or of a $G$-torsor on $X$. 


\section{Appendix}

\section{A. Two lemmas of Schlessinger}

In order to be as self-contained as possible, we include here the statements of two lemmas of Schlessinger, which play a key role in checking both Schlessinger's criterion and the deformation stack condition in several important examples. Although the proofs are not difficult, we do not reproduce them here.

Lemma A.1 (Schlessinger, Lemma 3.3 of [18]). Let $A$ be a ring, with nilpotent ideal $J$, and $u: M \rightarrow N$ a homomorphism of $A$-modules, with $N$ flat over $A$. If $\bar{u}: M / J M \rightarrow N / J N$ is an isomorphism, then $u$ is an isomorphism.

It follows easily that:

Corollary A.2. Let $M$ be a flat module over an Artin local ring $A$. Then $M$ is free.

Lemma A.3 (Schlessinger, Lemma 3.4 of [18]). Consider a commutative diagram

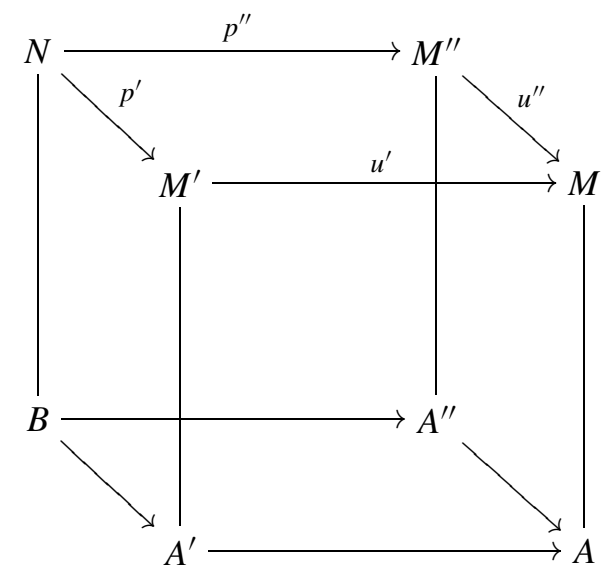

of compatible ring and module homomorphisms, where $B=A^{\prime} \times_{A} A^{\prime \prime}, N=$ $M^{\prime} \times{ }_{M} M^{\prime \prime}$, and $M^{\prime}$ and $M^{\prime \prime}$ are flat over $A^{\prime}$ and $A^{\prime \prime}$, respectively. Suppose

(i) $A^{\prime \prime} \rightarrow A$, with nilpotent kernel,

(ii) $u^{\prime}$ induces $M^{\prime} \otimes_{A^{\prime}} A \stackrel{\sim}{\rightarrow} M$, and similarly for $u^{\prime \prime}$.

Then $N$ is flat over $B$, and $p^{\prime}$ induces $N \otimes_{B} A^{\prime} \stackrel{\sim}{\rightarrow} M^{\prime}$, and similarly for $p^{\prime \prime}$.

\section{B. Hypercovers and $H^{2}$}

In this appendix we describe how to use the simplest non-trivial aspect of hypercovers (the general theory is due to Verdier, in [2, Section 7 of Expose V ]) to describe 
the sheaf cohomology group $H^{2}$ on an arbitrary topological space. The basic idea of hypercovers is quite simple: instead of fixing an open cover $\left\{U_{i}\right\}$ of a space $X$ and working only on the intersections of the various $U_{i}$, one allows further refinements at each stage, taking covers of each $U_{i} \cap U_{j}$, and so forth.

We begin by defining the type of covers we work with, using terminology consistent with that of Beke [4]:

Definition B.1. Given a topological space $X$, a cover $\mathcal{U}$ of level 2 of $X$ consists of an open cover $\left\{U_{i}\right\}_{i \in I}$ of $X$, together with the data of a cover $\left\{U_{i_{0}, i_{1}, j}\right\}_{j \in J_{i_{0}, i_{1}}}$ of each $U_{i_{0}} \cap U_{i_{1}}$, with $i_{0} \neq i_{1}$.

Note that for the sake of simplicity, the data of a cover of level 2 does not include different covers for $U_{i_{0}} \cap U_{i_{1}}$ and for $U_{i_{1}} \cap U_{i_{0}}$; by the same token, we take the cover of $U_{i_{0}} \cap U_{i_{0}}$ to be the single open set $U_{i_{0}}$, and denote this by an underscore. For instance, $U_{i_{0}, i_{0},-}=U_{i_{0}}$ is the unique open set of the cover of $U_{i_{0}} \cap U_{i_{0}}$.

Associated to $\mathcal{U}$ and a sheaf $\mathcal{F}$, we will define $H^{2}(\mathcal{U}, \mathcal{F})$, a cohomology group (in fact, a module over $\Gamma\left(X, \mathcal{O}_{X}\right)$ ) which directly generalizes Čech cohomology, and our main purpose is to prove the following very special case of Verdier's work:

Theorem B.2. Given any ringed space $\left(X, \mathcal{O}_{X}\right)$ and an $\mathcal{O}_{X}$-module $\mathcal{F}$, and any $\mathcal{U}$ a cover of level 2 of $X$, there exists a natural homomorphism of $\Gamma\left(X, \mathcal{O}_{X}\right)$-modules $H^{2}(\mathcal{U}, \mathcal{F}) \rightarrow H^{2}(X, \mathcal{F})$. phism

Furthermore, this map is compatible with refinement, and induces an isomor-

$$
\underset{\overrightarrow{\mathcal{U}}}{\lim } H^{2}(\mathcal{U}, \mathcal{F}) \stackrel{\sim}{\rightarrow} H^{2}(X, \mathcal{F})
$$

Here, a refinement of a cover of level 2 is defined in the natural way. Formally, it is:

Definition B.3. Let $\mathcal{U}$ be a cover of level 2 of $X$. A refinement $\mathcal{U}^{\prime}$ of $\mathcal{U}$ is another cover of level 2 of $X$, together with order-preserving maps of index sets $\pi: I^{\prime} \rightarrow I$ and for each $i_{0}^{\prime}<i_{1}^{\prime} \in I^{\prime}$ maps of index sets $\pi_{i_{0}^{\prime}, i_{1}^{\prime}}: J_{i_{0}^{\prime}, i_{1}^{\prime}} \rightarrow J_{\pi\left(i_{0}^{\prime}\right), \pi\left(i_{1}^{\prime}\right)}$, such that $U_{i^{\prime}}^{\prime} \subseteq U_{\pi\left(i^{\prime}\right)}$ for each $i^{\prime} \in I^{\prime}$, and $U_{i_{0}^{\prime}, i_{1}^{\prime}, j^{\prime}} \subseteq U_{\pi\left(i_{0}^{\prime}\right), \pi\left(i_{1}^{\prime}\right), \pi_{i_{0}^{\prime}, i_{1}^{\prime}}\left(j^{\prime}\right)}$ for each $j^{\prime} \in J_{i_{0}^{\prime}, i_{1}^{\prime}}^{\prime}$.

Note that covers of level 2 form a directed set under refinement, so it makes sense to take direct limits over all covers of level 2 of a given space.

As an immediate corollary of the theorem, we obtain the same statements for cohomology of sheaves of abelian groups on any topological space. We give the statement for ringed spaces because the proof is the same, and we will want to know that our $k$-linear structure is preserved by the isomorphism.

The definition of the group $H^{2}(\mathcal{U}, \mathcal{F})$ is the following. Note that unlike [8], we make use of unordered Cech cohomology rather than alternating Cech cohomology.

Definition B.4. Given an $\mathcal{O}_{X}$-module $\mathcal{F}$ on $X$, and $\mathcal{U}$ a cover of level 2 of $X$, we make the following definitions: $C^{1}(\mathcal{U}, \mathcal{F})$ is the $\Gamma\left(X, \mathcal{O}_{X}\right)$-module of 1-cochains

$$
\prod_{i_{0}, i_{1} \in I, j \in J_{i_{0}, i_{1}}} \mathcal{F}\left(U_{i_{0}, i_{1}, j}\right),
$$


and $C^{2}(\mathcal{U}, \mathcal{F})$ is the $\Gamma\left(X, \mathcal{O}_{X}\right)$-module of 2-cochains

$$
\begin{gathered}
\prod_{\mathbf{i}=\left(i_{0}, i_{1}, i_{2}\right) \in I^{3}} \mathcal{F}\left(U_{\mathbf{i}, \mathbf{j}}\right), \\
\mathbf{j}=\left(j_{1,2}, j_{0,2}, j_{0,1}\right) \in J_{i_{1}, i_{2}} \times J_{i_{0}, i_{2}} \times J_{i_{0}, i_{1}}
\end{gathered}
$$

where $U_{\mathbf{i}, \mathbf{j}}:=U_{i_{1}, i_{2}, j_{1,2}} \cap U_{i_{0}, i_{2}, j_{0,2}} \cap U_{i_{0}, i_{1}, j_{0,1}}$.

Then $Z^{2}(\mathcal{U}, \mathcal{F})$ is the submodule consisting of cocycles, which is to say $\rho \in$ $C^{2}(\mathcal{U}, \mathcal{F})$ satisfying the condition that for any $\mathbf{i}^{\prime}=\left(i_{0}, i_{1}, i_{2}, i_{3}\right) \in I$ and $\mathbf{j}^{\prime}:=$ $\left\{j_{m, m^{\prime}} \in J_{i_{m}, i_{m^{\prime}}}\right\}_{m, m^{\prime} \in\{0,1,2,3\}}$ we have

$$
\begin{aligned}
0=(d \rho)_{\mathbf{i}^{\prime}, \mathbf{j}^{\prime}}:= & \left.\rho_{i_{1}, i_{2}, i_{3}, j_{2,3}, j_{1,3}, j_{1,2}}\right|_{U_{\mathbf{i}^{\prime}, \mathbf{j}^{\prime}}}-\left.\rho_{i_{0}, i_{2}, i_{3}, j_{2,3}, j_{0,3}, j_{0,2}}\right|_{U_{\mathbf{i}^{\prime}, \mathbf{j}^{\prime}}} \\
& +\left.\rho_{i_{0}, i_{1}, i_{3}, j_{1,3}, j_{0,3}, j_{0,1}}\right|_{U_{\mathbf{i}^{\prime}, \mathbf{j}^{\prime}}}-\left.\rho_{i_{0}, i_{1}, i_{2}, j_{1,2}, j_{0,2}, j_{0,1}}\right|_{U_{\mathbf{i}^{\prime}, \mathbf{j}^{\prime}}} .
\end{aligned}
$$

Next, we define $B^{2}(\mathcal{U}, \mathcal{F})$ as the submodule of $Z^{2}(\mathcal{U}, \mathcal{F})$ consisting of coboundaries. These are the elements obtainable from a 1-cochain $\rho^{\prime} \in C^{1}(\mathcal{U}, \mathcal{F})$ by setting $\rho_{\mathbf{i}, \mathbf{j}}=\left(d \rho^{\prime}\right)_{\mathbf{i}, \mathbf{j}}:=\left.\rho_{i_{1}, i_{2}, j_{1,2}}^{\prime}\right|_{U_{\mathbf{i}, \mathbf{j}}}-\left.\rho_{i_{0}, i_{2}, j_{0,2}}^{\prime}\right|_{U_{\mathbf{i}, \mathbf{j}}}+\left.\rho_{i_{0}, i_{1}, j_{0,1}}^{\prime}\right|_{U_{\mathbf{i}, \mathbf{j}}}$

Finally, $H^{2}(\mathcal{U}, \mathcal{F})$ is defined to be $Z^{2}(\mathcal{U}, \mathcal{F}) / B^{2}(\mathcal{U}, \mathcal{F})$.

Note that in the special case that each cover $\left\{U_{i_{0}, i_{1}, j}\right\}_{j}$ consists of the single set $U_{i_{0}} \cap U_{i_{1}}$, we recover precisely the usual definition of the Čech cohomology group $\check{H}^{2}\left(\left\{U_{i}\right\}, \mathcal{F}\right)$.

We take a very hands-on approach to proving the theorem. A key lemma is the following:

Lemma B.5. Suppose that $\mathcal{F}$ is flasque. Then $H^{2}(\mathcal{U}, \mathcal{F})=0$.

Proof. Suppose we have $\rho \in Z^{2}(\mathcal{U}, \mathcal{F})$. We will construct a certain coboundary $d \rho^{\prime} \in B^{2}(\mathcal{U}, \mathcal{F})$, and show that subtracting it from $\rho$ removes the dependence on refinements, so that we obtain an element of the standard Čech cohomology group $\check{H}^{2}\left(\left\{U_{i}\right\}, \mathcal{F}\right)$. Since $\mathcal{F}$ is flasque, this element is then a coboundary by standard Čech cohomology theory.

$\rho^{\prime}$ is constructed as follows. Fix $i_{0}, i_{1}$, and for each $j, j^{\prime} \in J_{i_{0}, i_{1}}$, consider

$$
\rho_{i_{0}, i_{0}, i_{1}, j, j^{\prime},-}-\rho_{i_{0}, i_{0}, i_{0,-,-,}} \in \mathcal{F}\left(U_{i_{0}, i_{1}, j} \cap U_{i_{0}, i_{1}, j^{\prime}}\right) .
$$

This yields a Čech 1-cochain for the cover $\mathcal{U}_{i_{0}, i_{1}}:=\left\{U_{i_{0}, i_{1}, j}\right\}_{j}$ of $U_{i_{0}} \cap U_{i_{1}}$, and in fact we obtain a 1-cocycle, as can be verified using the cocycle condition for $\rho$, evaluated at $\mathbf{i}=\left(i_{0}, i_{0}, i_{0}, i_{1}\right)$. Since $\mathcal{F}$ is flasque, the 1 -cocycle can be written as $d \rho^{i_{0}, i_{1}}$ for some 0 -cochain $\rho^{i_{0}, i_{1}}$ on $\mathcal{U}_{i_{0}, i_{1}}$. Having done this for all $i_{0}, i_{1}$, we then define $\rho^{\prime}$ by $\rho_{i_{0}, i_{1}, j}^{\prime}:=\rho_{j}^{i_{0}, i_{1}}$. Then $d \rho^{\prime}$ is our desired coboundary.

We next claim that $\rho^{\prime \prime}=\rho-d \rho^{\prime}$ is a standard Čech 2-cocycle, as desired. It suffices to show that for any $\mathbf{i}=\left(i_{0}, i_{1}, i_{2}\right)$ and $\mathbf{j}, \mathbf{j}^{\prime}$, we have $\left.\rho_{\mathbf{i}, \mathbf{j}}^{\prime \prime}\right|_{U_{\mathbf{i}, \mathbf{j}} \cap U_{\mathbf{i}, \mathbf{j}^{\prime}}}-$ 
$\left.\rho_{\mathbf{i}, \mathbf{j}^{\prime}}^{\prime \prime}\right|_{U_{\mathbf{i}, \mathbf{j}} \cap U_{\mathbf{i}, \mathbf{j}^{\prime}}}=0$. Expanding the expression we find that the left hand side is given by

$$
\rho_{\mathbf{i}, \mathbf{j}}-\rho_{\mathbf{i}, \mathbf{j}^{\prime}}+\rho_{i_{0}, i_{0}, i_{2}, j_{0,2}, j_{0,2}^{\prime},-}-\rho_{i_{0}, i_{0}, i_{1}, j_{0,1}, j_{0,1}^{\prime},-}-\rho_{i_{1}, i_{1}, i_{2}, j_{1,2}, j_{1,2}^{\prime},-}+\rho_{i_{1}, i_{1}, i_{1},,_{-,},-}
$$

One then checks that the desired statement follows from the cocycle condition on $\rho$, evaluated at $\left(i_{0}, i_{0}, i_{1}, i_{2}\right),\left(i_{0}, i_{1}, i_{1}, i_{2}\right)$, and $\left(i_{0}, i_{1}, i_{1}, i_{1}\right)$.

It is now relatively straightforward to prove the theorem.

Proof of theorem. We begin by constructing the asserted natural map. Let

$$
\mathcal{F} \rightarrow \mathcal{G}_{0} \stackrel{d_{0}}{\rightarrow} \mathcal{G}_{1} \stackrel{d_{1}}{\rightarrow} \mathcal{G}_{2} \stackrel{d_{2}}{\rightarrow} \mathcal{G}_{3} \rightarrow \ldots
$$

be a flasque resolution of $\mathcal{F}$. We compute everything in terms of this resolution, making the identification $H^{2}(X, \mathcal{F})=\operatorname{ker} d_{2} / \operatorname{im} d_{1}$. Given $\rho \in H^{2}(\mathcal{U}, \mathcal{F})$, and choosing a representative 2-cocycle for $\rho$, we obtain its image $\rho^{0} \in Z^{2}\left(\mathcal{U}, \mathcal{G}_{0}\right)$, which by the lemma is $d \tilde{\rho}^{0}$ for some $\tilde{\rho}^{0} \in C^{1}\left(\mathcal{U}, \mathcal{G}_{0}\right)$. Taking the image of $\tilde{\rho}^{0}$ in $\mathcal{G}_{1}$, we obtain $\rho^{1} \in C^{1}\left(\mathcal{U}, \mathcal{G}_{1}\right)$. We claim that in fact $\rho^{1}$ is a standard Čech 1 cocycle. We have $d \rho^{1}=d\left(d_{0}\left(\tilde{\rho}^{0}\right)\right)=d_{0}\left(d\left(\tilde{\rho}^{0}\right)\right)=d_{0}\left(\rho^{0}\right)=0$, since $\rho^{0}$ consists of sections of $\mathcal{F}$. Thus, $\rho^{1}$ satisfies the cocycle condition, and is a standard Čech 1 -cocycle if and only if for any $i_{0} \neq i_{1}$, and $j, j^{\prime} \in J_{i_{0}, i_{1}}$, we have

$$
\left.\rho_{i_{0}, i_{1}, j}^{1}\right|_{U_{i_{0}, i_{1}, j} \cap U_{i_{0}, i_{1}, j^{\prime}}}-\left.\rho_{i_{0}, i_{1}, j^{\prime}}^{1}\right|_{U_{i_{0}, i_{1}, j} \cap U_{i_{0}, i_{1}, j^{\prime}}}=0 .
$$

However, setting $\mathbf{i}=\left(i_{0}, i_{0}, i_{1}\right)$ and $\mathbf{j}=\left(j, j^{\prime}{ }_{-}{ }_{-}\right)$, and evaluating

$$
0=\left(d \rho^{1}\right)_{i_{0}, i_{0}, i_{1}, j, j^{\prime},-}=\left.\rho_{i_{0}, i_{1}, j}^{1}\right|_{U_{\mathbf{i}, \mathbf{j}}}-\left.\rho_{i_{0}, i_{1}, j^{\prime}}^{1}\right|_{U_{\mathbf{i}, \mathbf{j}}}+\left.\rho_{i_{0}, i_{0},-}^{1}\right|_{U_{\mathbf{i}, \mathbf{j}}}
$$

we need only see that $\rho_{i_{0}, i_{0},-}^{1}=0$, which follows from evaluating $0=\left(d \rho^{1}\right)_{i_{0}, i_{0}, i_{0,-,-,}}$ $=\rho_{i_{0}, i_{0},-}^{1}$. We therefore have that $\rho^{1}$ is a Čech 1-cocycle, and again by standard Čech cohomology we conclude that $\rho^{1}=d \tilde{\rho}^{1}$ for some Čech 0 -cochain $\tilde{\rho}^{1}$ on $\left\{U_{i}\right\}$ with coefficients in $\mathcal{G}_{1}$. Finally taking the image of $\tilde{\rho}^{1}$ in $\mathcal{G}_{2}$, we see that we get a global section $\rho^{2}$ of $\mathcal{G}_{2}$, since the differences on $U_{i_{0}} \cap U_{i_{1}}$ take values in $\mathcal{G}_{0}$. Moreover, this global section lies in ker $d_{2}$, since it is locally in the image of $d_{1}$. Taking the class of $\rho^{2}$ in $H^{2}(X, \mathcal{F})$ constructs the desired map $H^{2}(\mathcal{U}, \mathcal{F}) \rightarrow$ $H^{2}(X, \mathcal{F})$.

It remains to check that this map is a well-defined homomorphism, compatible with refinement, and bijective in the limit. For the map to be well defined, we need to note that if $\rho$ is modified by a 2 -coboundary of $\mathcal{F}$, we will have $\tilde{\rho}^{0}$ modified by a 1 -cochain in the image of $\mathcal{F}$, which then vanishes after taking the image in $\mathcal{G}_{1}$, and similarly if $\tilde{\rho}^{0}$ is modified by a Čech 1-cocycle (equivalently, coboundary) of $\mathcal{G}_{0}$, we will modify $\tilde{\rho}^{1}$ by a 0 -cochain in the image of $\mathcal{G}_{0}$, which vanishes in $\mathcal{G}_{2}$. Finally, if $\tilde{\rho}^{1}$ is modified by a 0 -cocycle of $\mathcal{G}_{1}$, then its image in ker $d_{2}$ is modified 
by an element of im $d_{1}$, leaving the class in $H^{2}(X, \mathcal{F})$ unaffected. We also see easily that the map we have defined is a module homomorphism, since each of the $d_{i}$ are homomorphisms. Compatibility with refinement from $\mathcal{U}$ to $\mathcal{U}^{\prime}$ is clear, since at each stage we can choose $\rho, \rho^{0}$, and $\rho^{1}$ on $\mathcal{U}^{\prime}$ to be obtained by refinement from $\mathcal{U}$, and the image $\rho^{2}$ in $\mathcal{G}_{2}$ will then be unchanged.

Injectivity and surjectivity in the limit then follow by explicit construction: if we have for some cover $\mathcal{U}$ of level 2 an element $\rho \in H^{2}(\mathcal{U}, \mathcal{F})$ having image 0 in $H^{2}(X, \mathcal{F})$, we have that the image of $\tilde{\rho}^{1}$ in $\mathcal{G}_{2}$ agrees with the image of some global section $s_{\rho} \in \Gamma\left(\mathcal{G}_{1}\right)$, so that $\tilde{\rho}_{i}^{1}-\left.s_{\rho}\right|_{U_{i}}$ maps to 0 in $\mathcal{G}_{2}$, and after possible refinement of $\mathcal{U}$, is the image of some 0 -cochain of $\mathcal{G}_{0}$. Thus, $\tilde{\rho}^{1}$ is the sum of a 0 -cocycle of $\mathcal{G}_{1}$ and 0 -cochain of $\mathcal{G}_{0}$, which means that $\rho^{1}$ is the image of a 1 -coboundary of $\mathcal{G}_{0}$, and hence that $\tilde{\rho}^{0}$ is the sum of a 1-coboundary of $\mathcal{G}_{0}$ and a 1-cochain in the kernel of $d_{0}$. The latter cochain is in the image of $\mathcal{F}$ after refining $\mathcal{U}$ once more, so we find that $\rho^{0}$ is the image of a 2-coboundary of $\mathcal{F}$, so that we have a refinement $\mathcal{U}^{\prime}$ of $\mathcal{U}$ on which $\rho$ vanishes, as desired.

Finally, surjectivity in the limit is proved by starting with $\xi \in H^{2}(X, \mathcal{F})$, represented as an element of $\operatorname{ker} d_{2} \subseteq \Gamma\left(\mathcal{G}_{2}\right)$, and choosing $\left\{U_{i}\right\}$ to be a cover of $X$ on which $\xi$ is the image of some $\xi_{i} \in \mathcal{G}_{1}\left(U_{i}\right)$. Then for each $i_{0} \neq i_{1}$, we let $\left\{U_{i_{0}, i_{1}, j}\right\}_{j}$ be a cover of $U_{i_{0}} \cap U_{i_{1}}$ on which $\xi_{i_{1}}-\xi_{i_{0}}$ is the image of some $\tilde{\rho}^{0}{ }_{i_{0}, i_{1}, j} \in \mathcal{G}_{0}\left(U_{i_{0}, i_{1}, j}\right)$. This gives us a $\tilde{\rho}^{0} \in C^{1}\left(\mathcal{U}, \mathcal{G}_{0}\right)$, and taking $\rho^{0}=d \tilde{\rho}^{0}$ we see that every section $\rho_{\mathbf{i}, \mathbf{j}}^{0}$ is in the kernel of $d_{0}$, so after possible further refinement, is the image of some $\rho_{\mathbf{i}, \mathbf{j}} \in \mathcal{F}\left(U_{\mathbf{i}, \mathbf{j}}\right)$. We have thus constructed $\rho \in Z^{2}(\mathcal{U}, \mathcal{F})$, with image $\xi \in H^{2}(X, \mathcal{F})$, as desired.

We conclude with an example demonstrating that all of this was actually necessary, even for very reasonable (by algebrogeometric standards!) topological spaces. See also the discussion preceding the proof of Proposition 3.12.

Example B.6. We let $X$ be the topological space underlying the scheme $\mathbb{A}_{k}^{2}$, where $k$ is any field. We produce a presheaf $\mathcal{F}$ of abelian groups such that the sheafification $\tilde{\mathcal{F}}$ is the constant sheaf associated to $\mathbb{Z}$, but there is no open cover $\left\{U_{i}\right\}$ of $X$ with sections $\rho_{i} \in \mathcal{F}\left(U_{i}\right)$ such that each $\rho_{i}$ maps to 1 in $\tilde{F}\left(U_{i}\right)$ and $\left.\rho_{i}\right|_{U_{i} \cap U_{j}}=\left.\rho_{j}\right|_{U_{i} \cap U_{j}}$ in $\mathcal{F}\left(U_{i} \cap U_{j}\right)$.

We construct $\mathcal{F}$ as follows. Let $V_{1}=\mathbb{A}_{k}^{2} \backslash\{(0,0)\}$, and $V_{2}=\mathbb{A}_{k}^{2} \backslash\{(1,0)\}$. Let $\mathcal{V}$ be the collection of open subsets of $\mathbb{A}_{k}^{2}$ whose complement contains an irreducible curve through $(0,0)$ and $(1,0)$. We then define:

$$
\mathcal{F}(U)= \begin{cases}0: & U \nsubseteq V_{1} \text { and } U \nsubseteq V_{2} ; \\ \mathbb{Z} \times 0: & U \subseteq V_{1} \text { and } U \nsubseteq V_{2} ; \\ 0 \times \mathbb{Z}: & U \subseteq V_{2} \text { and } U \nsubseteq V_{1} \\ \mathbb{Z} \times \mathbb{Z}: & U \subseteq V_{1} \cap V_{2} \text { and } U \notin \mathcal{V} \\ \mathbb{Z}: & U \in \mathcal{V} .\end{cases}
$$


Restriction maps $\mathbb{Z} \times \mathbb{Z} \rightarrow \mathbb{Z}$ are given by the summation map, while the remaining restriction maps are the obvious ones.

We note that $\mathcal{V}$ is an open cover of $V_{1} \cap V_{2}$, since we can for instance remove the curves $y=0$ or $y=x(x-1)$, whose intersection is precisely $(0,0)$ and $(1,0)$. It is then easy to check that $\mathcal{F}$ is a presheaf with constant sheafification $\tilde{\mathcal{F}}=\underline{\mathbb{Z}}$, and that if $\left\{U_{i}\right\}$ is any cover of $X$ with $\rho_{i} \in \mathcal{F}\left(U_{i}\right)$ mapping to 1 in $\tilde{\mathcal{F}}$ for all $i$, if we choose $U_{i}$ containing $(0,0)$ and $U_{j}$ containing $(1,0)$, then we must have $\left.\rho_{i}\right|_{U_{i} \cap U_{j}} \neq\left.\rho_{j}\right|_{U_{i} \cap U_{j}}$. Specifically, we check that we must have $U_{i} \subseteq V_{2}$ but $U_{i} \nsubseteq V_{1}$ and $U_{j} \subseteq V_{1}$ but $U_{j} \nsubseteq V_{2}$, and that $U_{i} \cap U_{j} \notin \mathcal{V}$, from which the claim clearly follows.

\section{References}

[1] M. ARTin, Versal deformations and algebraic stacks, Invent. Math. 27 (1974), 165-189.

[2] M. Artin, A. Grothendieck and J.-L. Verdier, "Théorie des Topos et Cohomologie Étale des Schémas", Tome 2, SGA, no. 4, Springer-Verlag, 1972.

[3] M. Artin, A. Grothendieck and J.-L. Verdier, "Théorie des Topos et Cohomologie Étale des Schémas", Tome 3, SGA, no. 4, Springer-Verlag, 1973.

[4] T. BEKE, Higher Čech theory, $K$-theory 32 (2004), 293-322.

[5] B. FANTECHI and L. GÖTTSCHE, Local properties and Hilbert schemes of points, In: "Fundamental Algebraic Geometry, Mathematical Surveys and Monographs", Vol. 123, American Mathematical Society, 2005, pp. 139-178.

[6] A. Grothendieck and J. Dieudonné, "Éléments de Géométrie Algébrique: IV. Étude Locale des Schémas et des Morphismes de Schémas, quatriéme partie", Publ. Math. Inst. Hautes Études Sci., Vol. 32, Institut des Hautes Études Scientifiques, 1967.

[7] A. Grothendieck, "Catégories Cofibrées Additives et Complexe Cotangent Relatif", Lecture Notes in Mathematics, Vol. 79, Springer-Verlag, 1968.

[8] R. HARTSHORne, "Algebraic Geometry", Springer-Verlag, 1977.

[9] R. HARTShorne, "Deformation Theory", Graduate Texts in Mathematics, Vol. 257, Springer-Verlag, 2010.

[10] D. Huybrechts and M. Lehn, "The Geometry of Moduli Spaces of Sheaves", Fried. Vieweg \& Sohn, Braunschweig, 1997.

[11] L. ILlusiE, "Complexe Cotangent et Déformations. I", Lecture Notes in Mathematics, Vol. 239, Springer-Verlag, 1971.

[12] S. LiChtENBAUM and M. SChlessinger, The cotangent complex of a morphism, Trans. Amer. Math. Soc. 128 (1967), 41-70.

[13] H. Matsumura, "Commutative Ring Theory”, Cambridge University Press, 1986.

[14] M. OLSSON and J. STARR, Quot functors for Deligne-Mumford stacks, Comm. Algebra 31 (2003), 4069-4096, special volume in honor of S. Kleiman's 60th birthday.

[15] M. C. Olsson, Crystalline cohomology of stacks and Hyodo-Kato cohomology, Astérisque 316, (2007).

[16] B. Osserman, Deformations and automorphisms: triangles of deformation problems, in preparation.

[17] D. S. RIM, "Formal Deformation Theory", Groupes de monodromie en géométrie algébrique, Lecture Notes in Mathematics, Vol. 288, Springer-Verlag, 1972, expose VI.

[18] M. Schlessinger, Functors of Artin rings, Tran. Amer. Math. Soc. 130 (1968), 208-222.

[19] J.-P. SERRE, "Local Fields", Springer-Verlag, 1979.

[20] A. Vistoli, The deformation theory of local complete intersections, preprint. 
[21] V. Voevodsky, Homotopy theory of simplicial sheaves in completely decomposable topologies, J. Pure Appl. Algebra 214 (2010), 1384-1398.

[22] V. Voevodsky, Unstable motivic homotopy categories in Nisnevich and cdh-topologies, J. Pure Appl. Algebra 214 (2010), 1399-1406.

Department of Mathematics

University of California at Davis

One Shields Ave.

Davis, CA 95616 USA

osserman@math.ucdavis.edu 